\title{
On the Origin of Fluorescence from Boranils in the Crystalline Phase
}

\author{
Hatun H. T. Al-Sharif, ${ }^{\text {a }}$ Raymond Ziessel, ${ }^{\text {a }}$ Paul G. Waddell, ${ }^{\mathrm{b}}$ Casey Dixon ${ }^{\mathrm{c}}$ and \\ Anthony Harriman*,a \\ (a) Molecular Photonics Laboratory, School of Natural and Environmental Sciences, Bedson \\ Building, Newcastle University, Newcastle upon Tyne, NE1 7RU, UK. \\ (b) Crystallography Laboratory, School of Natural and Environmental Sciences, Bedson \\ Building, Newcastle University, Newcastle upon Tyne, NE1 7RU, UK. \\ (c) NMR Laboratory, School of Natural and Environmental Sciences, Bedson Building, \\ Newcastle University, Newcastle upon Tyne, NE1 7RU, UK.
}

\section{SUPPORTING INFORMATION - TOTAL PAGES 54}

\section{Table of contents:}

S1. Experimental procedures S2

S2. Atom numbering system used for X-ray crystallography S26

$\begin{array}{lll}\text { S3. Structural details generated by computation } & \text { S27 }\end{array}$

S4. Effect of solvent on the photophysical properties S33

S5. Emission from the solid-state S39

S6. Electronic energy transfer in crystalline media S46

S7. Effect of increased temperature for a thin PMMA film S50

S8. Drop cast films $\quad$ S51

$\begin{array}{lll}\text { S9. } & \text { References } & \text { S53 }\end{array}$ 


\section{S1. Experimental procedures}

Samples: Samples of $\mathrm{B}(\mathrm{I})$ and $\mathrm{B}\left(\mathrm{NO}_{2}\right)$ were prepared as before $\mathrm{S}^{\mathrm{S} 1}$ and purified by column chromatography on silica gel. Samples were recrystallized from tetrahydrofuran / pentane layers and used for the X-ray structural studies. Examples of ${ }^{1} \mathrm{H}$ and ${ }^{13} \mathrm{C}$ NMR spectra recorded in $\mathrm{CDCl}_{3}$ are provided below. $A$ sample of $\mathrm{B}(\mathrm{H})$ was prepared by the same method starting from $\mathrm{N}, \mathrm{N}$-diethylaniline (1 equivalent) and 2-hydroxybenzaldehye (1-equivalent) in refluxing ethanol containing a trace amount of $\mathrm{p}-\mathrm{TsOH}$ as catalyst. After overnight reflux, the solution was cooled in ice and the resultant precipitate collected by filtration. The so-formed anil was washed with ice-cold ethanol and petroleum spirits before being dried under vacuum. The anil (1 equivalent) was dissolved in dry dichloroethane and treated with $\mathrm{BF}_{3} . \mathrm{Et}_{2} \mathrm{O}$ (2.5 equivalents) as carrier. After a short reflux period, a precipitate began to form and diisopropylethylamine (2.5 equivalents) as base was added. Reflux was continued and the course of reaction was followed by TLC. After cooling to room temperature, the solution was washed with saturated aqueous $\mathrm{NaHCO}_{3}$ solution and subsequently extracted with $\mathrm{CH}_{2} \mathrm{Cl}_{2}$. The organic layer was dried over $\mathrm{MgSO}_{4}$ and evaporated to dryness under vacuum. The solid was purified by column chromatography on silica gel to give a pale yellow powder. A sample was recrystallized from tetrahydrofuran with a top layer of pentane. This latter procedure was repeated to give diffraction quality yellow crystals. ${ }^{1} \mathrm{H}$ and ${ }^{13} \mathrm{C} N M R$ spectra were recorded in fully deuterated solvents at $298 \mathrm{~K}$ on a Bruker Avance III HD $500 \mathrm{MHz}$ or $700 \mathrm{MHz}$ spectrometers. Examples of ${ }^{1} \mathrm{H}$ and ${ }^{13} \mathrm{C}$ NMR spectra, together with 2D-NMR spectra and FTIR spectra for solid material, are shown below as Figures S1-S21. The various chemical shifts are summarized in Tables S1-S3.

Spectroscopic studies: Spectroscopic-grade solvents were purchased from commercial sources and used as received after ensuring the absence of fluorescent impurities. For all solution-phase studies, absorption spectra were recorded with a Hitachi U-3310 spectrophotometer while steady-state fluorescence studies were made with an Yvon-Jobin Fluorolog tau-4 spectrometer. Spectra were recorded at ambient temperature unless stated otherwise. All fluorescence spectra were recorded under optically dilute conditions and were supported by excitation spectra. Experimental data were extracted from the instrument and processed separately to provide reduced spectra for Gaussian analyses. ${ }^{\text {s2 }}$ Fluorescence quantum yields were determined by reference to known standards used at optically dilute 
concentrations. The main fluorescence standard was perylene in cyclohexane $\left(\Phi_{\mathrm{F}}=0.94 \pm\right.$ 0.02 ), with excitation at $370-420 \mathrm{~nm} .{ }^{\mathrm{S3}}$ Ancillary reference systems included Coumarin-153 in deaerated ethanol $\left(\Phi_{\mathrm{F}}=0.38 \pm 0.03\right) .{ }^{S 4}$ For the solvent-dependent studies, a concentrated solution of the boranil was prepared in $\mathrm{CH}_{2} \mathrm{Cl}_{2}$ and a standard aliquot was added to a calibrated volumetric flask. The solvent was removed with a stream of $d r y \mathrm{~N}_{2}$ and the required volume of the target solvent was added. Small corrections were made for any change in absorbance at the excitation wavelength. Corrections were made for changes in refractive index. ${ }^{55}$ Fluorescence lifetimes were recorded by time-correlated, single photon counting methods with high intensity, short duration laser diodes as excitation source. These diodes provided discrete excitation wavelengths of $375 \mathrm{~nm}$ (FWHM = $130 \mathrm{ps}), 420 \mathrm{~nm}$ (FWHM = 100 ps) and $515 \mathrm{~nm}$ (FWHM = $140 \mathrm{ps})$. Fluorescence was isolated from scattered excitation light using a high-radiance monochromator and detection was made with a micro-channel plate PMT operated at $-20{ }^{\circ} \mathrm{C}$. Data analysis ${ }^{56}$ was made by standard statistical methods. Temperature dependence studies were made with an Oxford Instruments Optistat DN using dilute solutions of the fluorophore in freshly distilled MTHF solution. Higher temperature studies were made with a Harricks' demountable liquid cell and temperature controller. In some cases, the crystals were ground with dried $\mathrm{KBr}$ and pressed into a compact disk under vacuum.

Spectroscopic studies made with crystalline materials were carried out using an integrating sphere (StellaNet Inc.) to house the sample. For emission studies, a notch filter was used to exclude excitation light and the output signal was directed to the spectrophotometer via optical fibres. For absorption spectra, a few crystals were deposited onto a clean quartz plate and spectra recorded using optical fibres to connect with the instrument. Fluorescence quantum yields were measured with the integrating sphere using the procedure recommended by Beeby et al. ${ }^{\mathrm{S7}}$ The quoted values are the average of 7-10 individual measurements. For the quantum yield studies, the excitation source was a low-power laser diode emitting at $405 \mathrm{~nm}$, operated at a series of emission intensities as modulated with neutral density filters. The same laser diode was used for simple polarization studies. The vertically polarized output beam, after passing through a polarizer, was used for excitation of the crystal. A second polarizer was placed before the entrance slit to the monochromator. Control experiments were made to correct for any stray polarization of the instrument. 
Structural studies: All crystal structural data were collected using copper radiation $(\lambda \mathrm{CuK} \alpha=$ $1.54184 \AA$ A) on an Xcalibur, Atlas, Gemini Ultra diffractometer equipped with an Oxford Cryosystems CryostreamPlus open-flow $\mathrm{N}_{2}$ cooling device. The intensities were corrected for absorption using a multifaceted crystal model created by indexing the faces of the crystal for which data were collected or treated empirically using spherical harmonics. Data were collected at 150K. Cell refinement, data collection and data reduction were undertaken via the software CrysAlisPro. ${ }^{\mathrm{S8}}$ Structures were solved using $\mathrm{XT}^{\mathrm{S9}}$ and refined by $\mathrm{XL}^{\mathrm{S10}}$ using the Olex2 interface. ${ }^{\mathrm{S11}}$ All non-hydrogen atoms were refined anisotropically and hydrogen atoms were positioned with idealized geometry. The displacement parameters of the hydrogen atoms were constrained using a riding model with $\mathrm{U}_{(\mathrm{H})}$ set to be an appropriate multiple of the $U_{\text {eq }}$ value of the parent atom.

CCDC information: $B(H)$ CCDC $1980928: B(I) C C D C 1980929: B\left(N_{2}\right)$ CCDC 1980930

Computational studies: Spectral deconstruction of reduced absorption or fluorescence spectra was made with PEAKFIT. The minimum number of Gaussian profiles needed to accurately reproduce the entire spectral envelop was used for the analysis. Quantum chemical calculations were made with TURBOMOLE. ${ }^{\mathrm{S} 12}$ Structural studies were made at the DFT level using the B3LYP functional and with the PBEO basis set. Solvent effects were modelled using the PCM approach with the Mennucci-Tomasi correction. ${ }^{S 13}$ Rotational studies were made by semi-empirical methods using AMPAC-10 and the COSMO model for solvent effects. ${ }^{\mathrm{S} 14}$ These calculations used the non-rigid chain method ${ }^{515}$ with the DFT optimized initial and final geometries. 


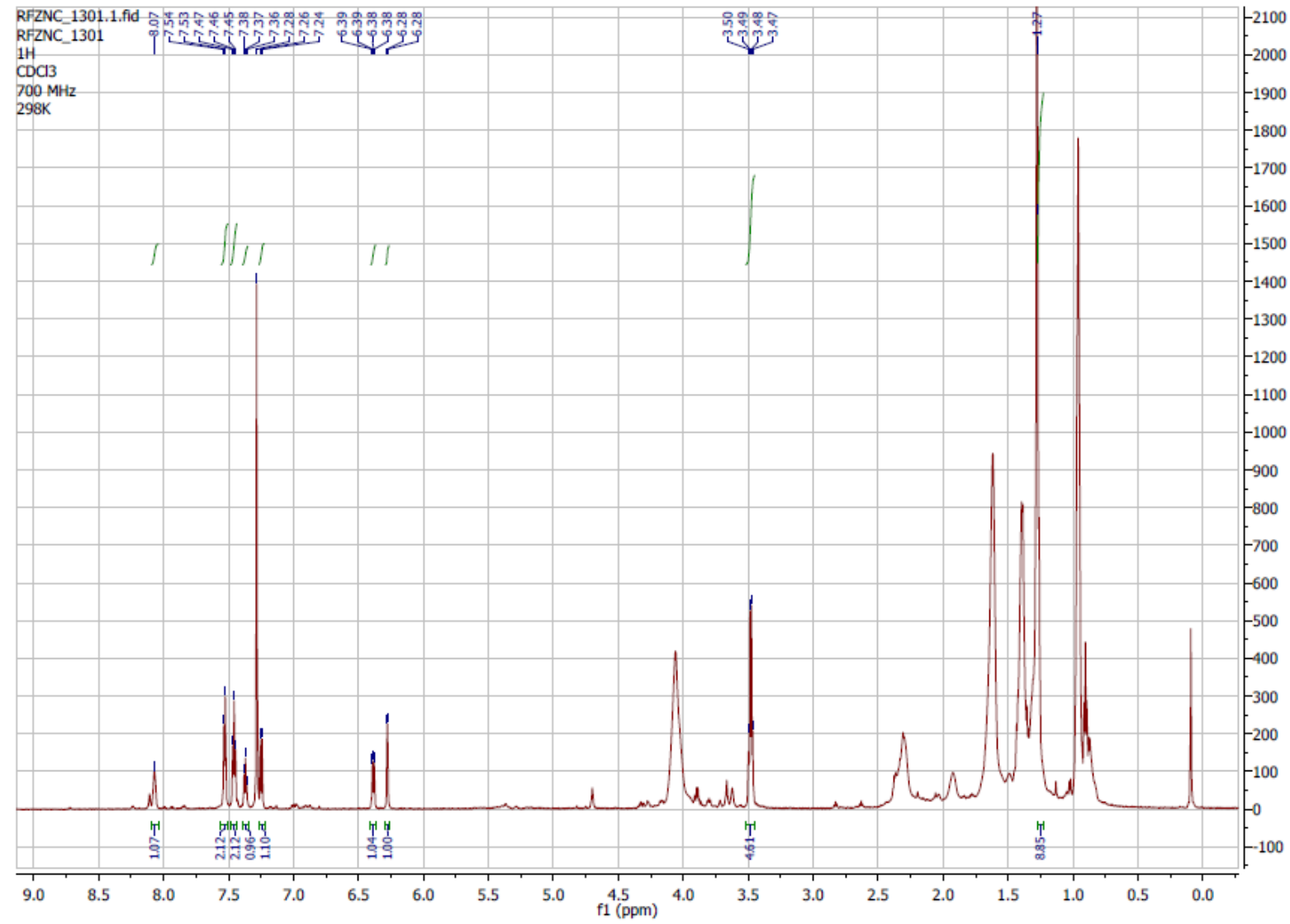

Figure $\mathrm{S} 1 .{ }^{1} \mathrm{H}-\mathrm{NMR}(700 \mathrm{MHz})$ spectrum recorded for $\mathrm{B}(\mathrm{H})$ in $\mathrm{CDCl}_{3}$ at room temperature. 


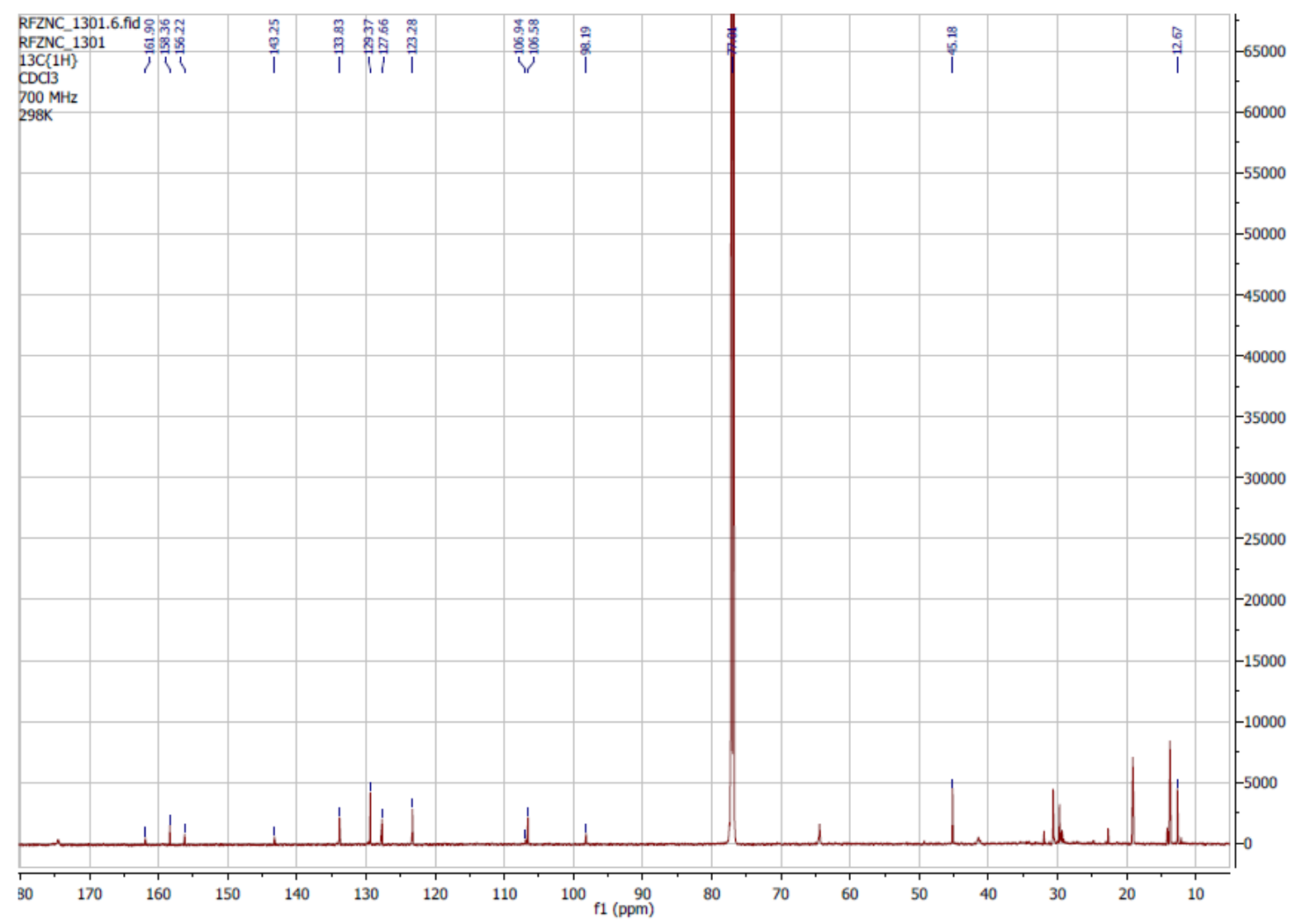

Figure S2. ${ }^{13} \mathrm{C} N M R(700 \mathrm{MHz})$ spectrum recorded for $\mathrm{B}(\mathrm{H})$ in $\mathrm{CDCl}_{3}$ at room temperature. 


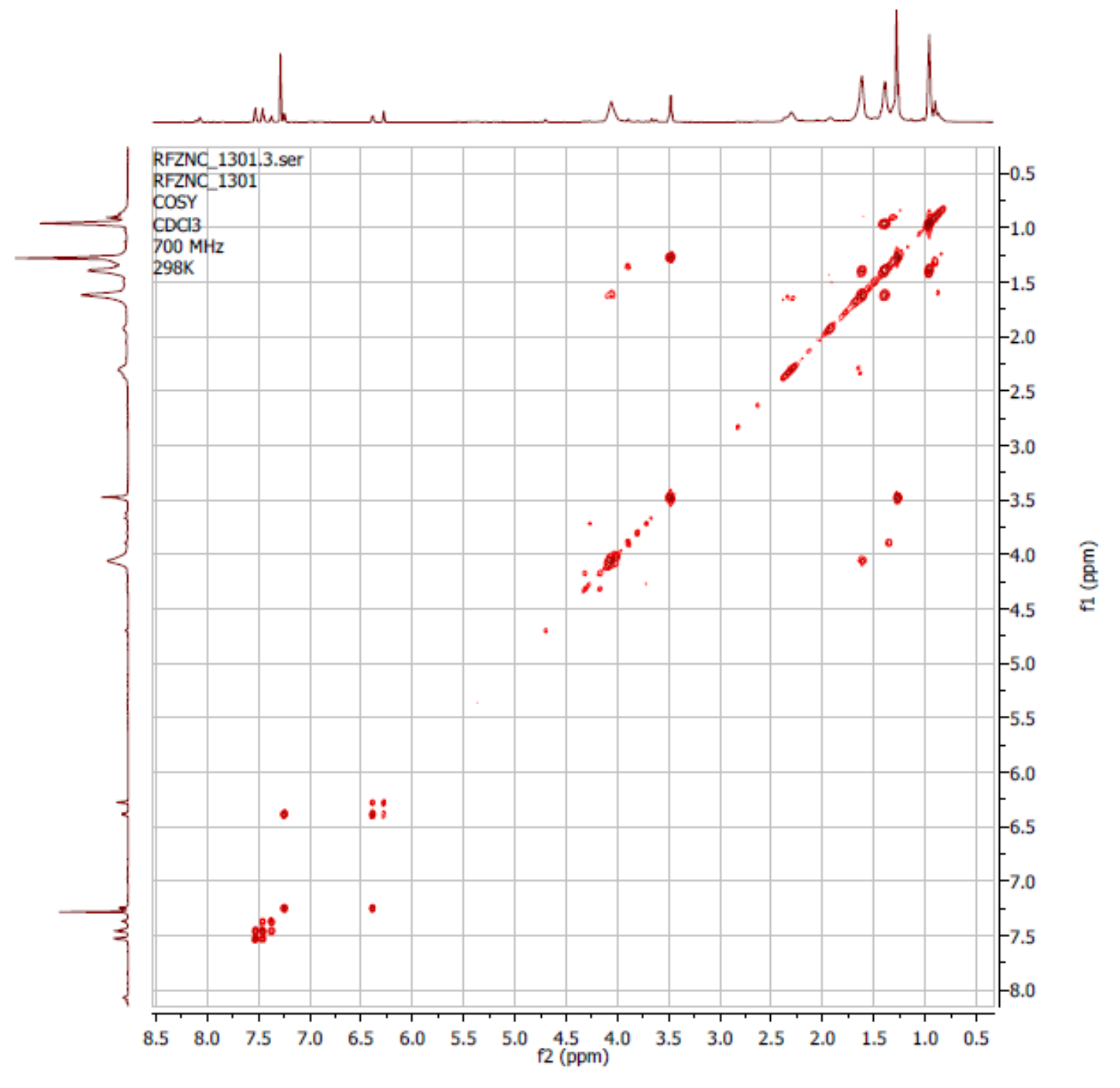

Figure S3. COSY $(700 \mathrm{MHz})$ spectrum recorded for $\mathrm{B}(\mathrm{H})$ in $\mathrm{CDCl}_{3}$ at room temperature. 


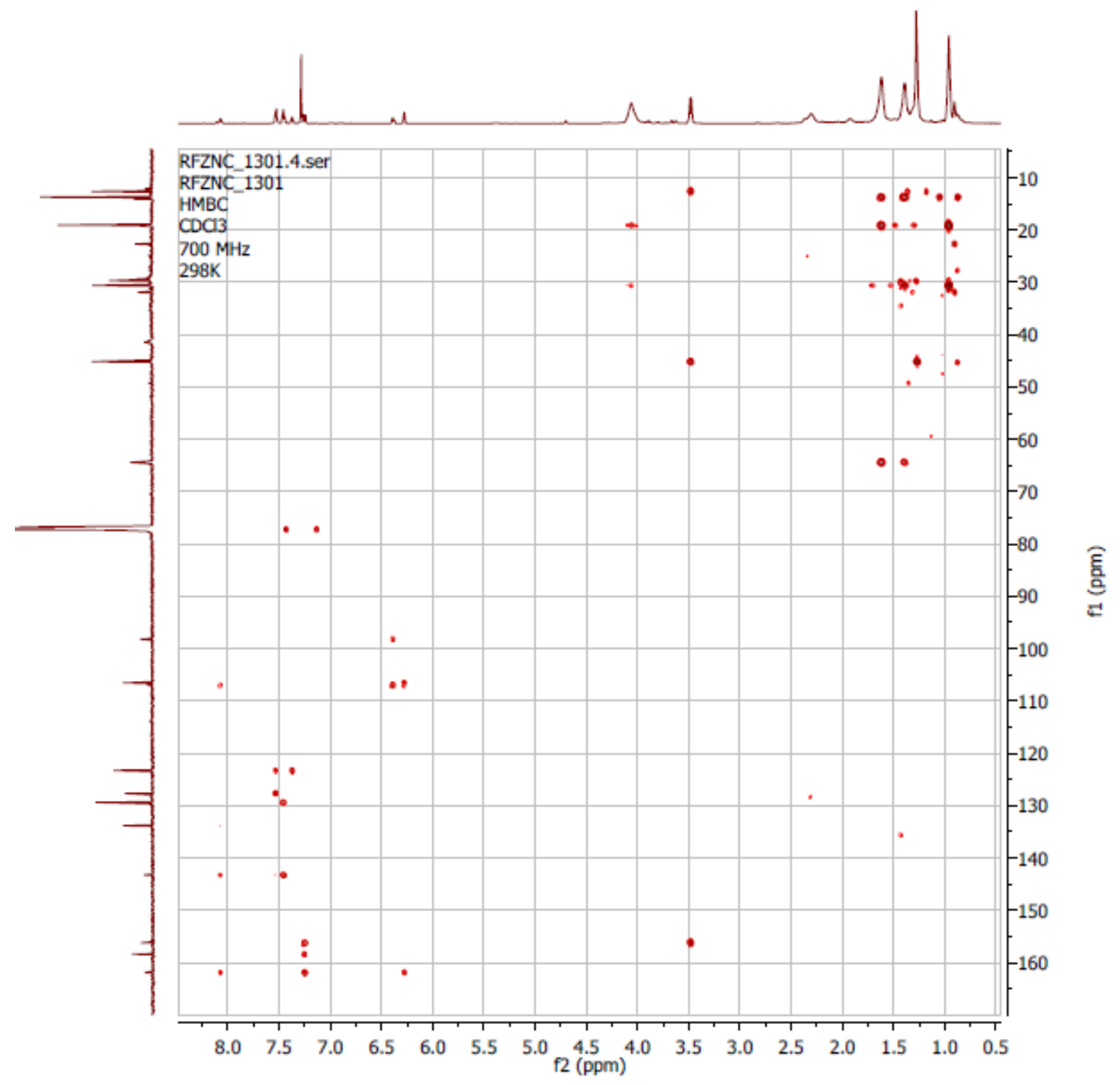

Figure S4. $\mathrm{HMBC}(700 \mathrm{MHz})$ spectrum recorded for $\mathrm{B}(\mathrm{H})$ in $\mathrm{CDCl}_{3}$ at room temperature. 


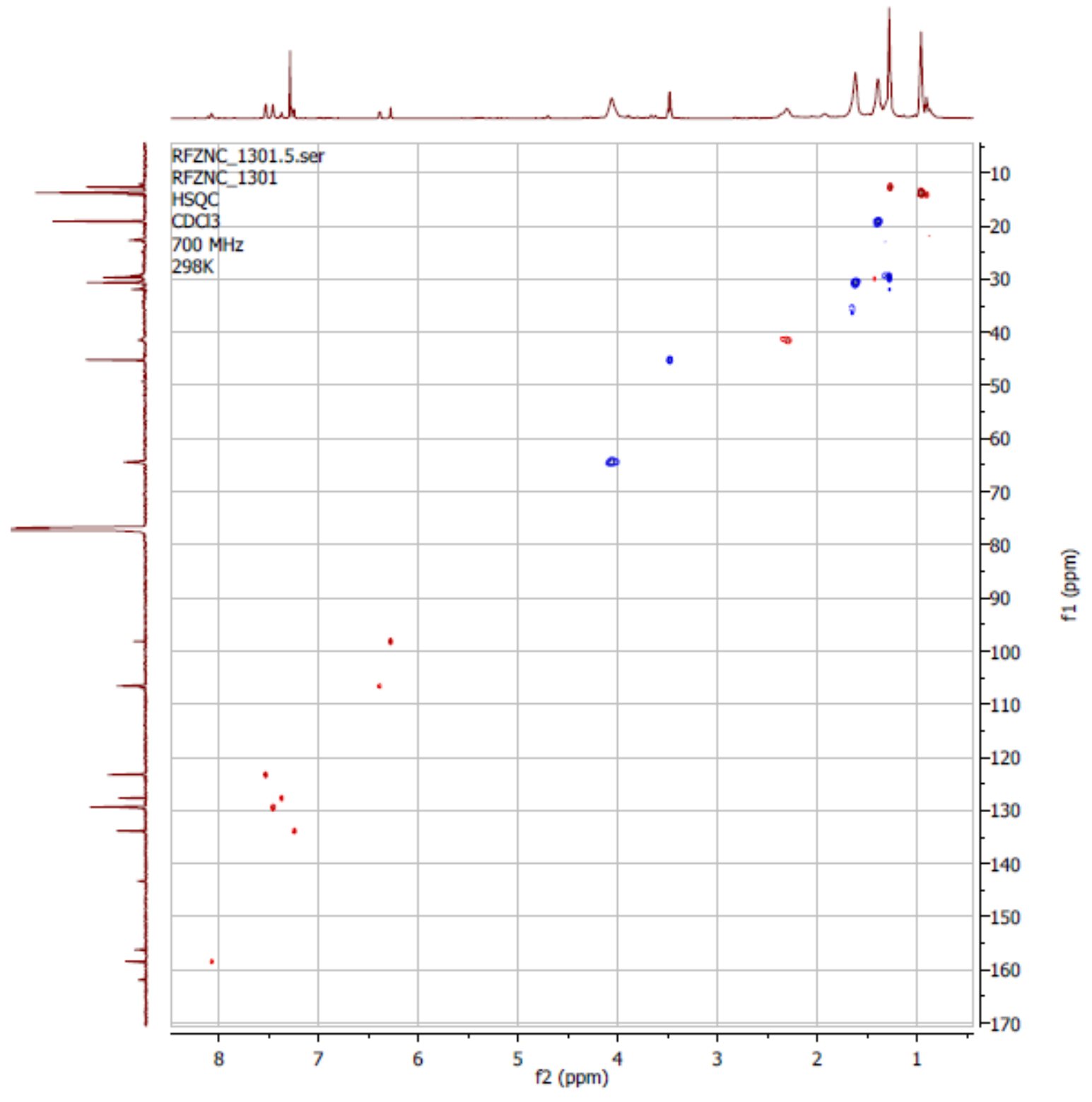

Figure S5. $\mathrm{HSQC}(700 \mathrm{MHz})$ spectrum recorded for $\mathrm{B}(\mathrm{H})$ in $\mathrm{CDCl}_{3}$ at room temperature. 


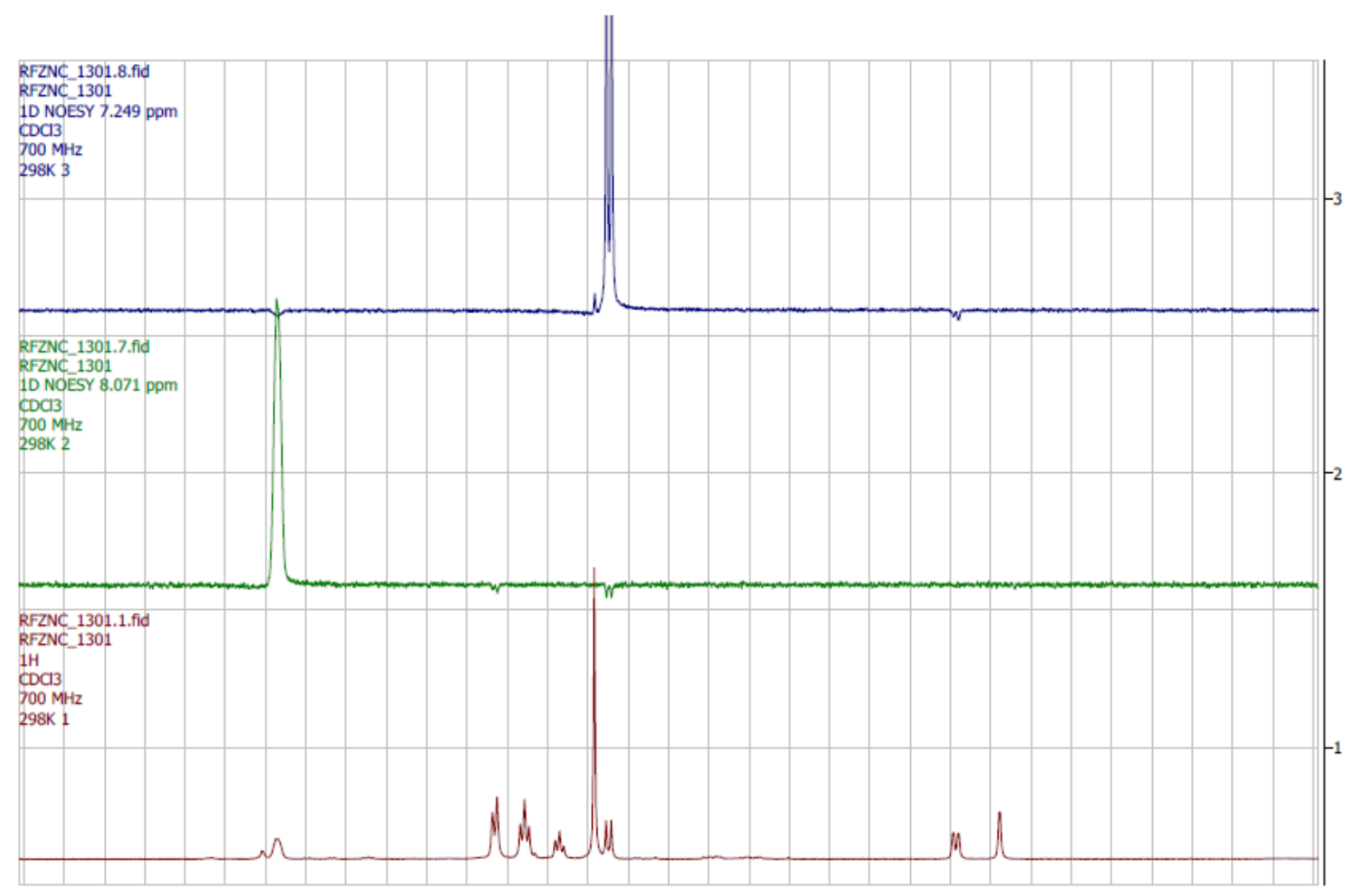

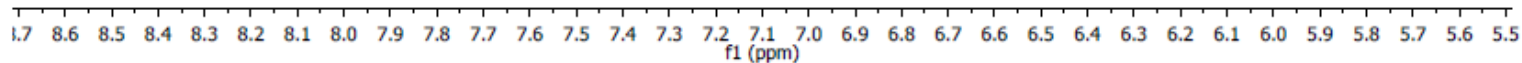

Figure S6. 1D-NOESY spectra recorded for $\mathrm{B}(\mathrm{H})$ in $\mathrm{CDCl}_{3}$ at room temperature. See Table $\mathrm{S} 1$ for assignments.

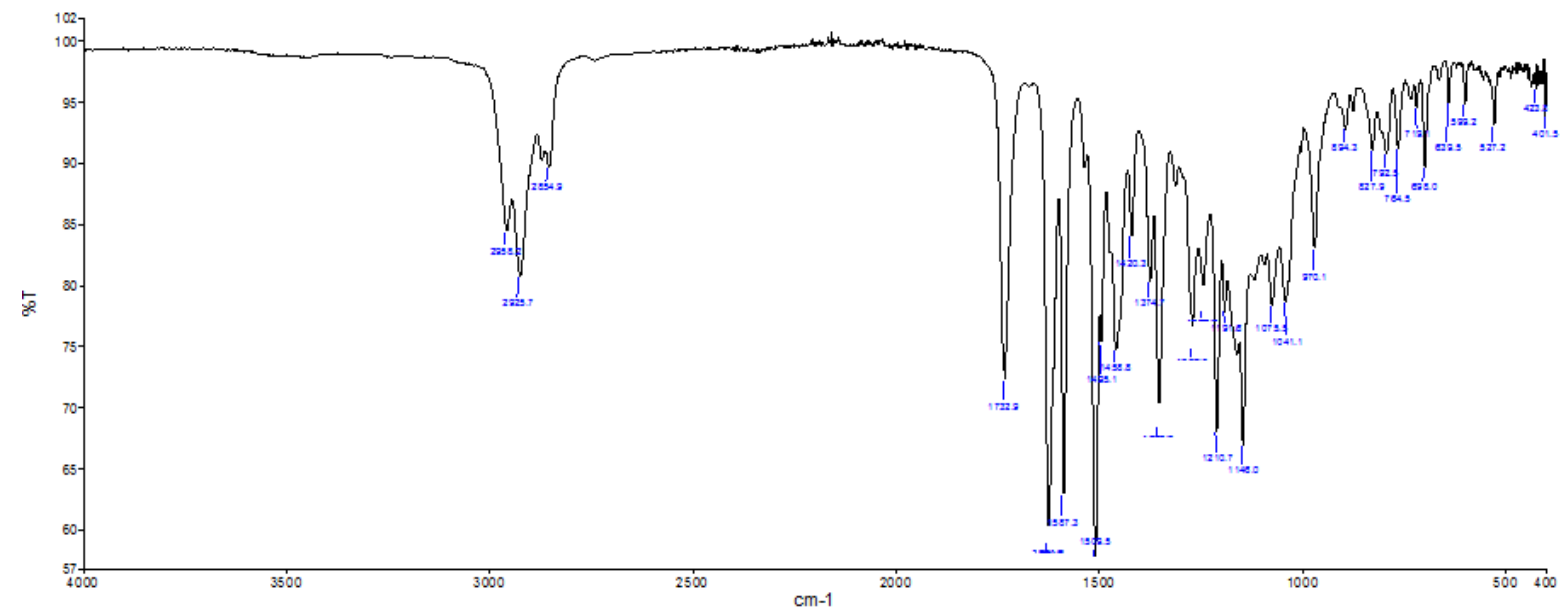

Figure S7. FT-IR spectrum recorded for $\mathrm{B}(\mathrm{H})$ in the solid state. 


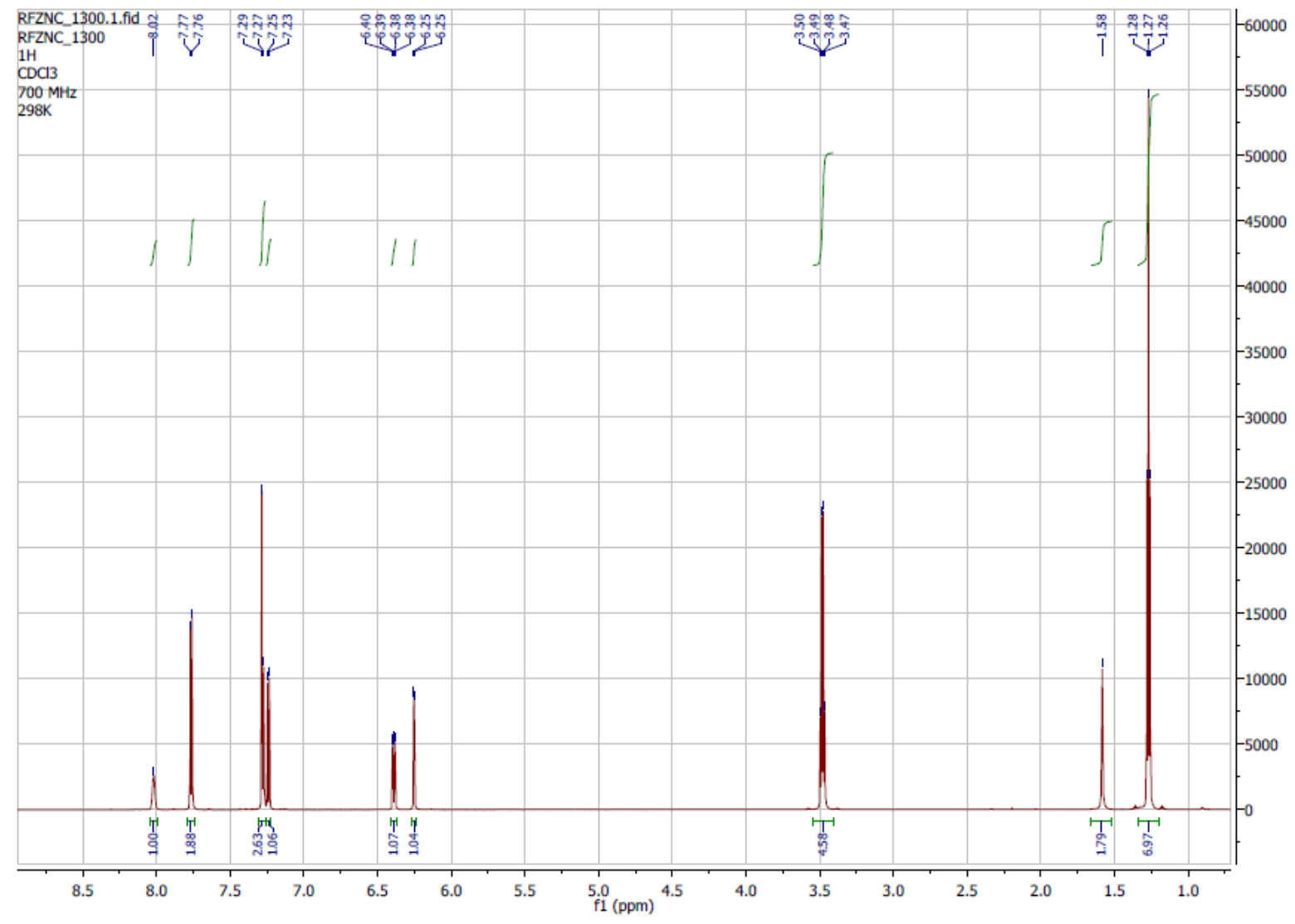

Figure S8. ${ }^{1} \mathrm{H}-\mathrm{NMR}(700 \mathrm{MHz})$ spectrum recorded for $\mathrm{B}(\mathrm{I})$ in $\mathrm{CDCl}_{3}$ at room temperature. 


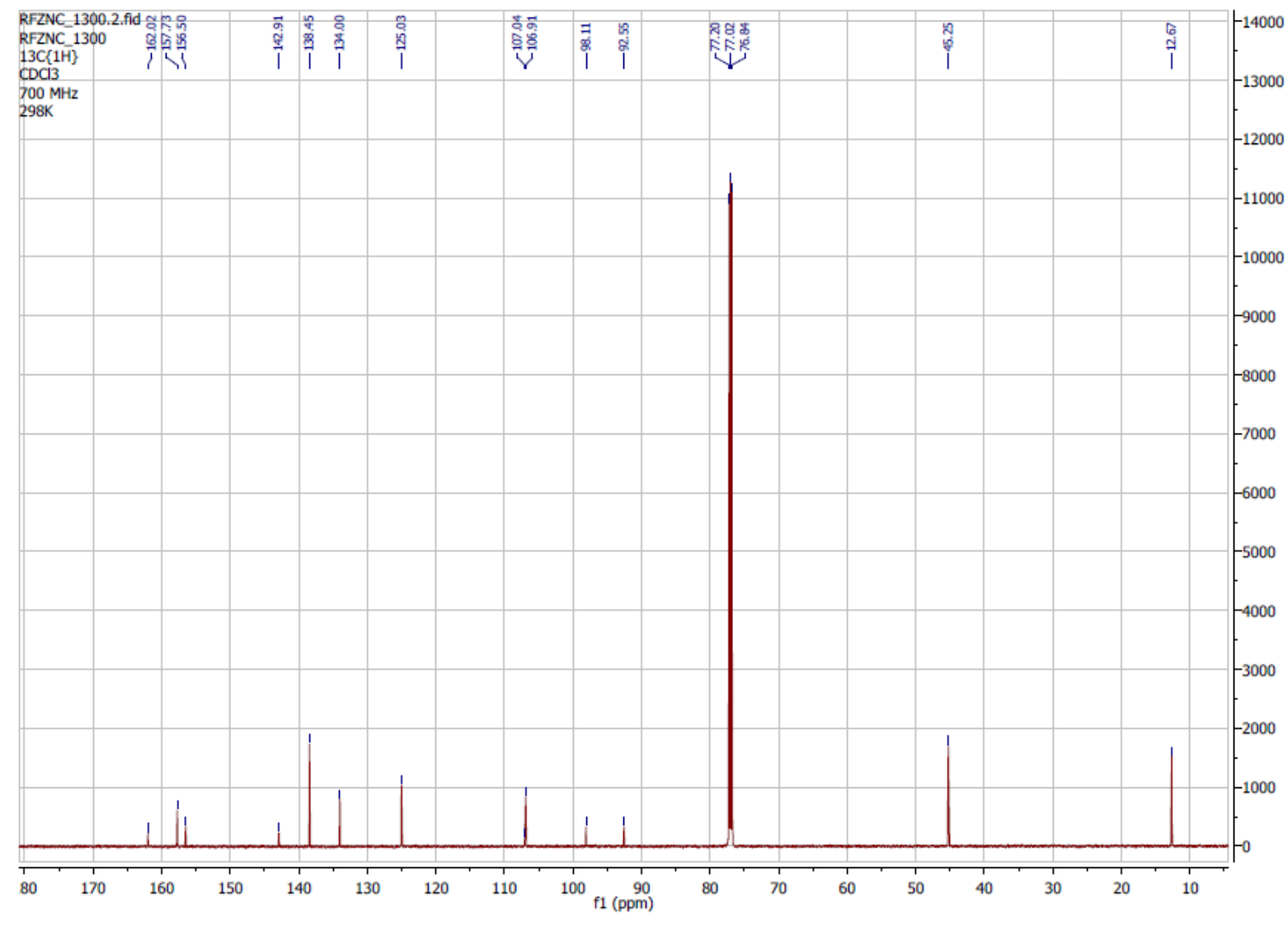

Figure S9. ${ }^{13} \mathrm{C}-\mathrm{NMR}(700 \mathrm{MHz})$ spectrum recorded for $\mathrm{B}(\mathrm{I})$ in $\mathrm{CDCl}_{3}$ at room temperature. 


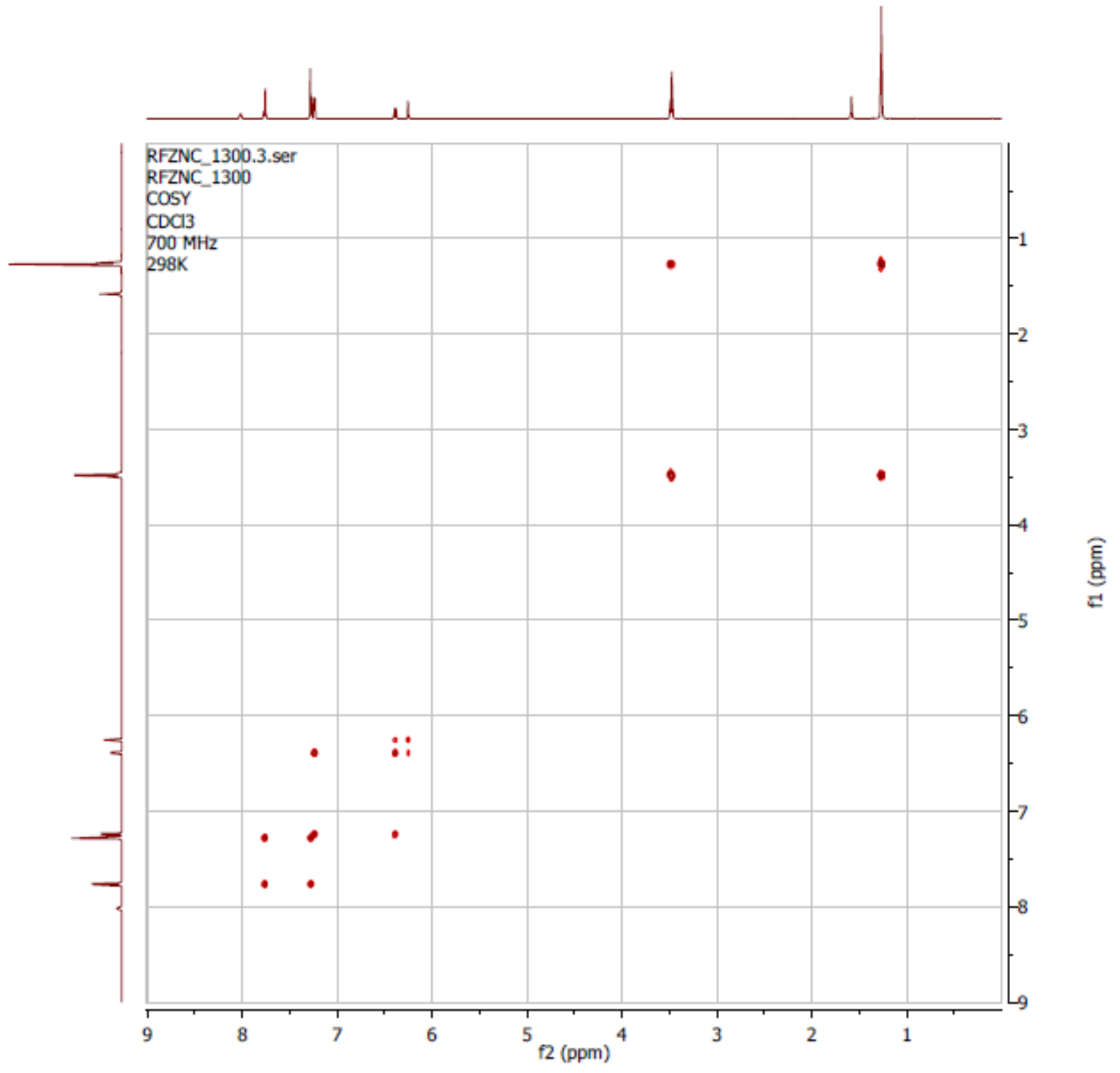

Figure S10. COSY-NMR (700 MHz) spectrum recorded for $\mathrm{B}(\mathrm{I})$ in $\mathrm{CDCl}_{3}$ at room temperature. 


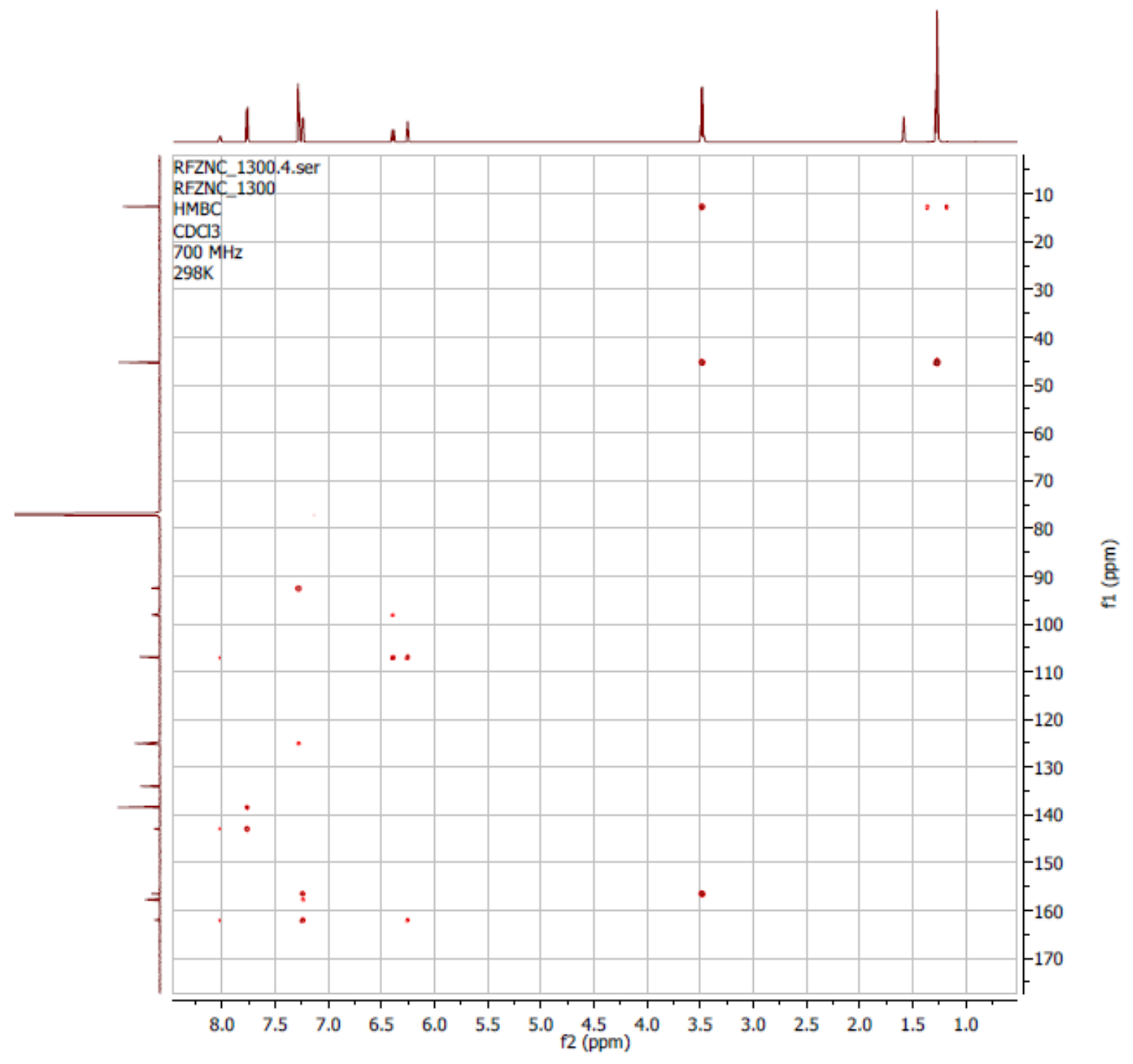

Figure S11. HMBC-NMR (700 MHz) spectrum recorded for $\mathrm{B}(\mathrm{I})$ in $\mathrm{CDCl}_{3}$ at room temperature. 


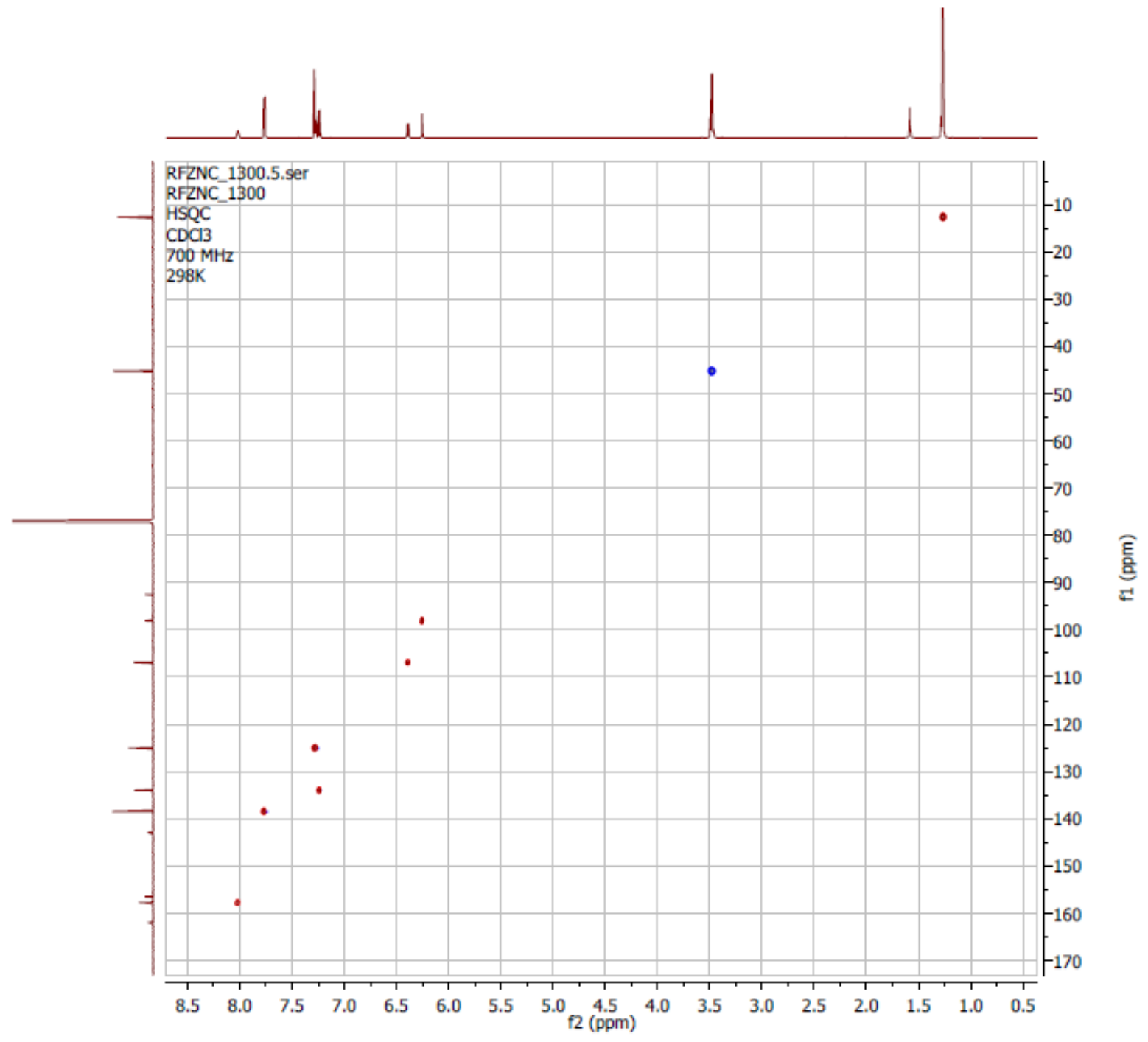

Figure S12. HSQC-NMR (700 MHz) spectrum recorded for $\mathrm{B}(\mathrm{I})$ in $\mathrm{CDCl}_{3}$ at room temperature.

S15 


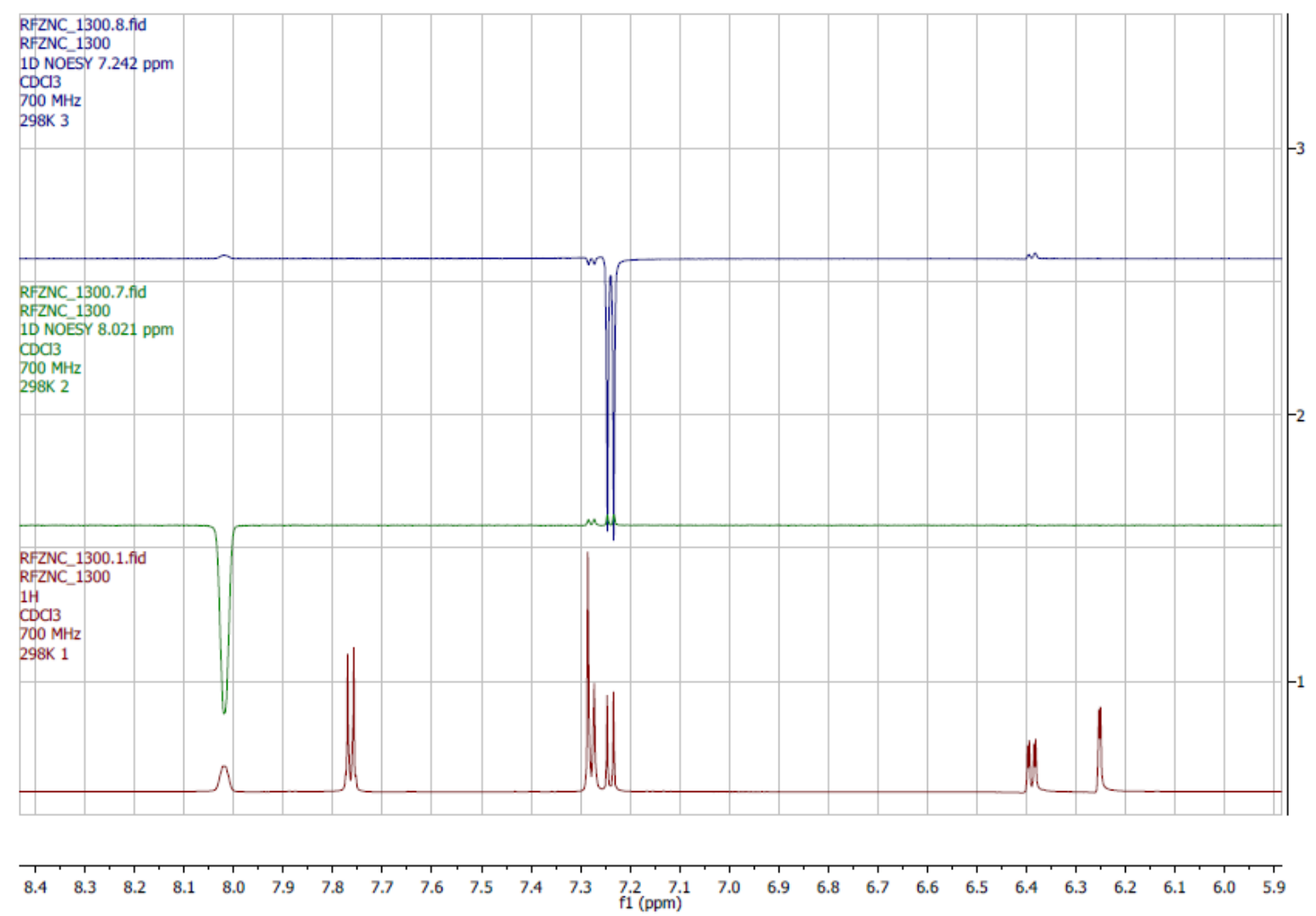

Figure S13. 1D-NOESY NMR (700 MHz) spectra recorded for $\mathrm{B}(\mathrm{I})$ in $\mathrm{CDCl}_{3}$ at room temperature. See Table S2 for assignments.

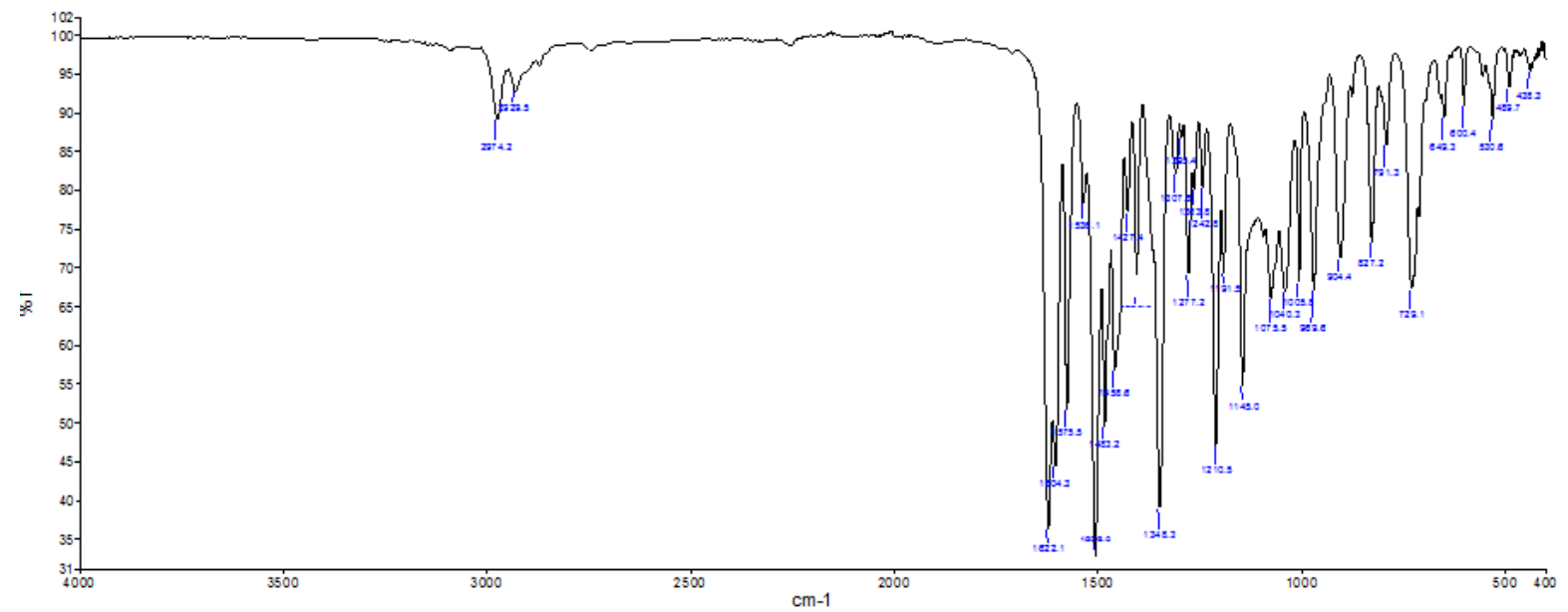

Figure S14. FT-IR spectrum recorded for B(I) in the solid state. 


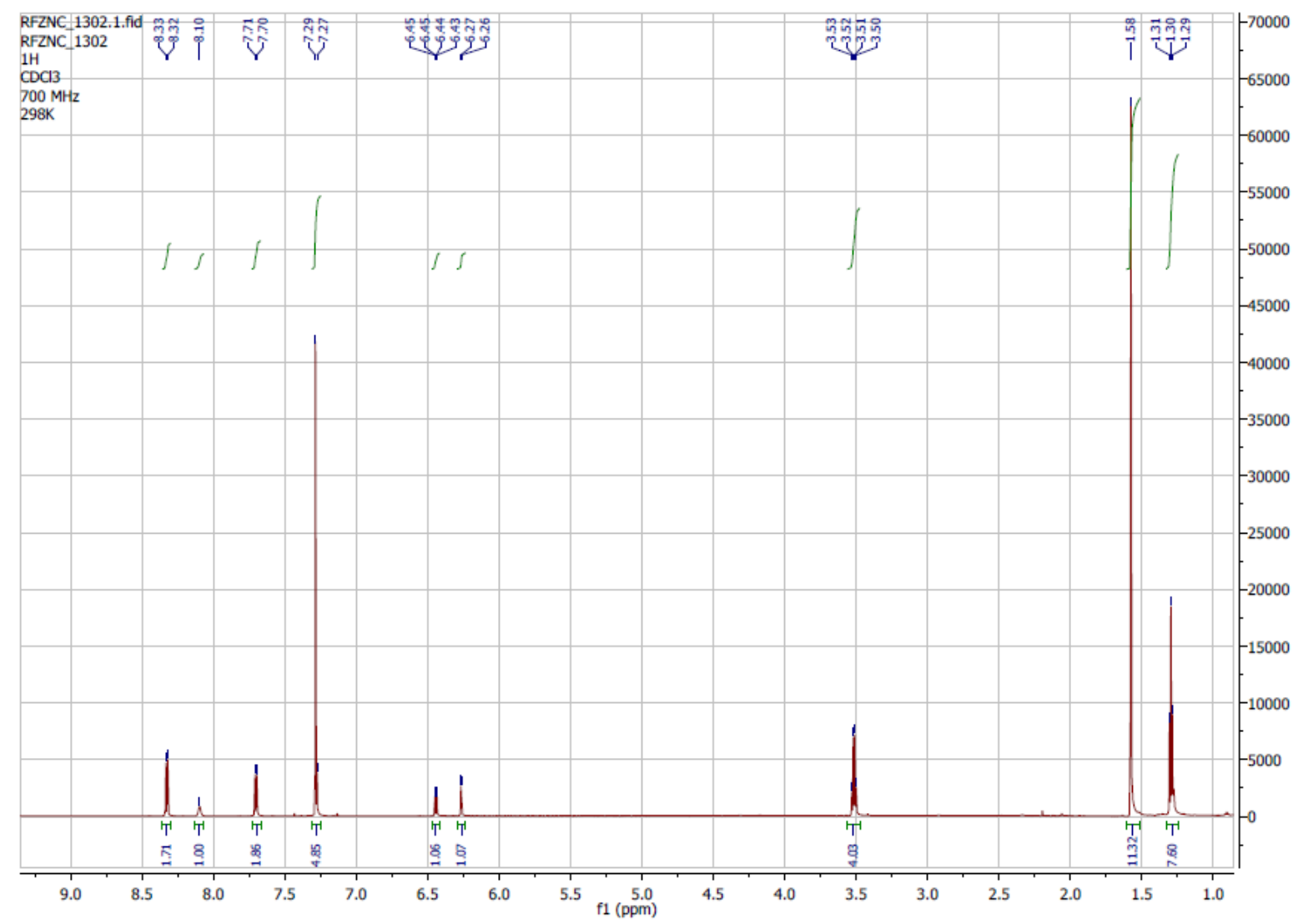

Figure S15. ${ }^{1} \mathrm{H}-\mathrm{NMR}(700 \mathrm{MHz})$ spectrum recorded for $\mathrm{B}\left(\mathrm{NO}_{2}\right)$ in $\mathrm{CDCl}_{3}$ at room temperature. 


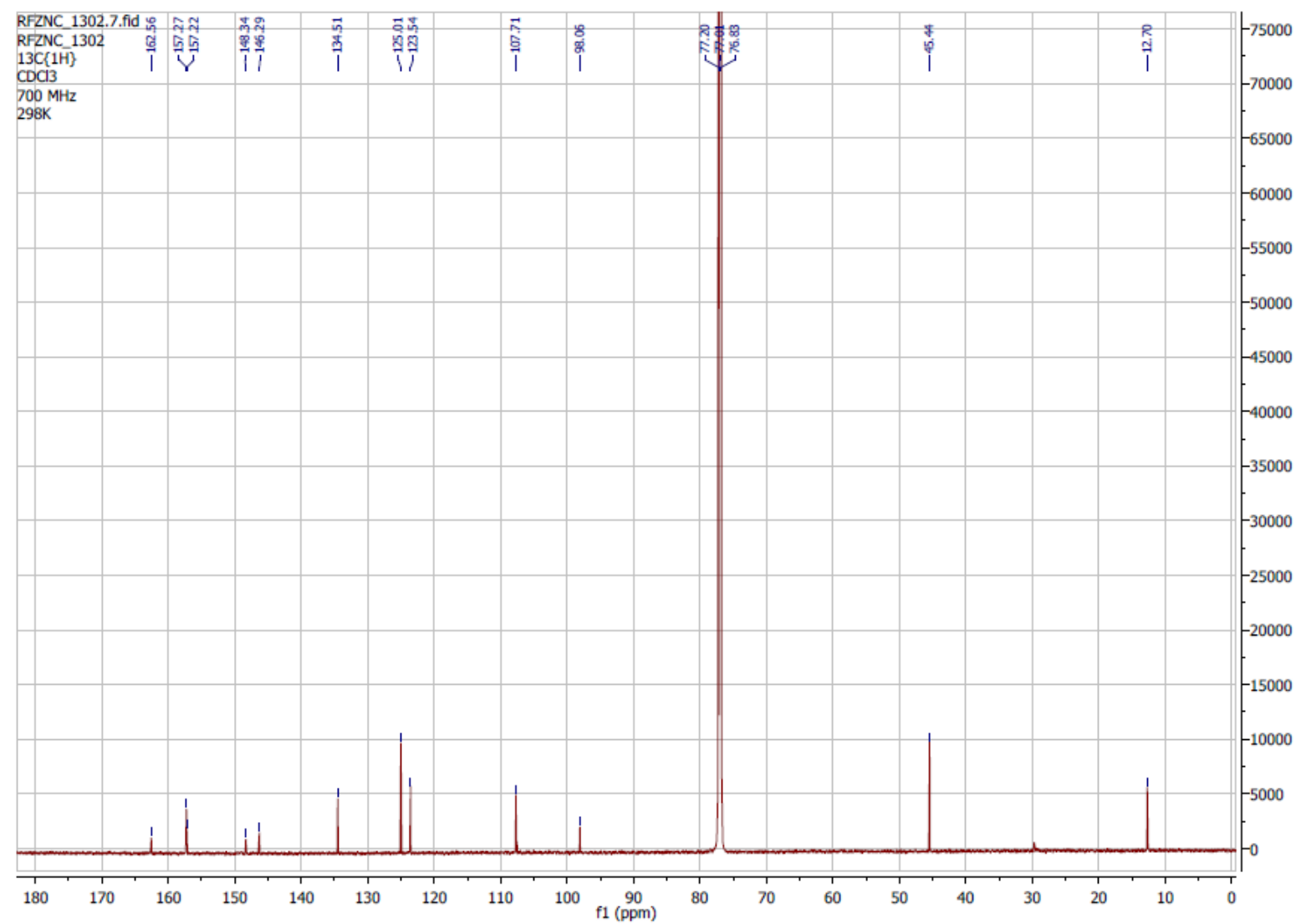

Figure S16. ${ }^{13} \mathrm{C}-\mathrm{NMR}(700 \mathrm{MHz})$ spectrum recorded for $\mathrm{B}\left(\mathrm{NO}_{2}\right)$ in $\mathrm{CDCl}_{3}$ at room temperature. 


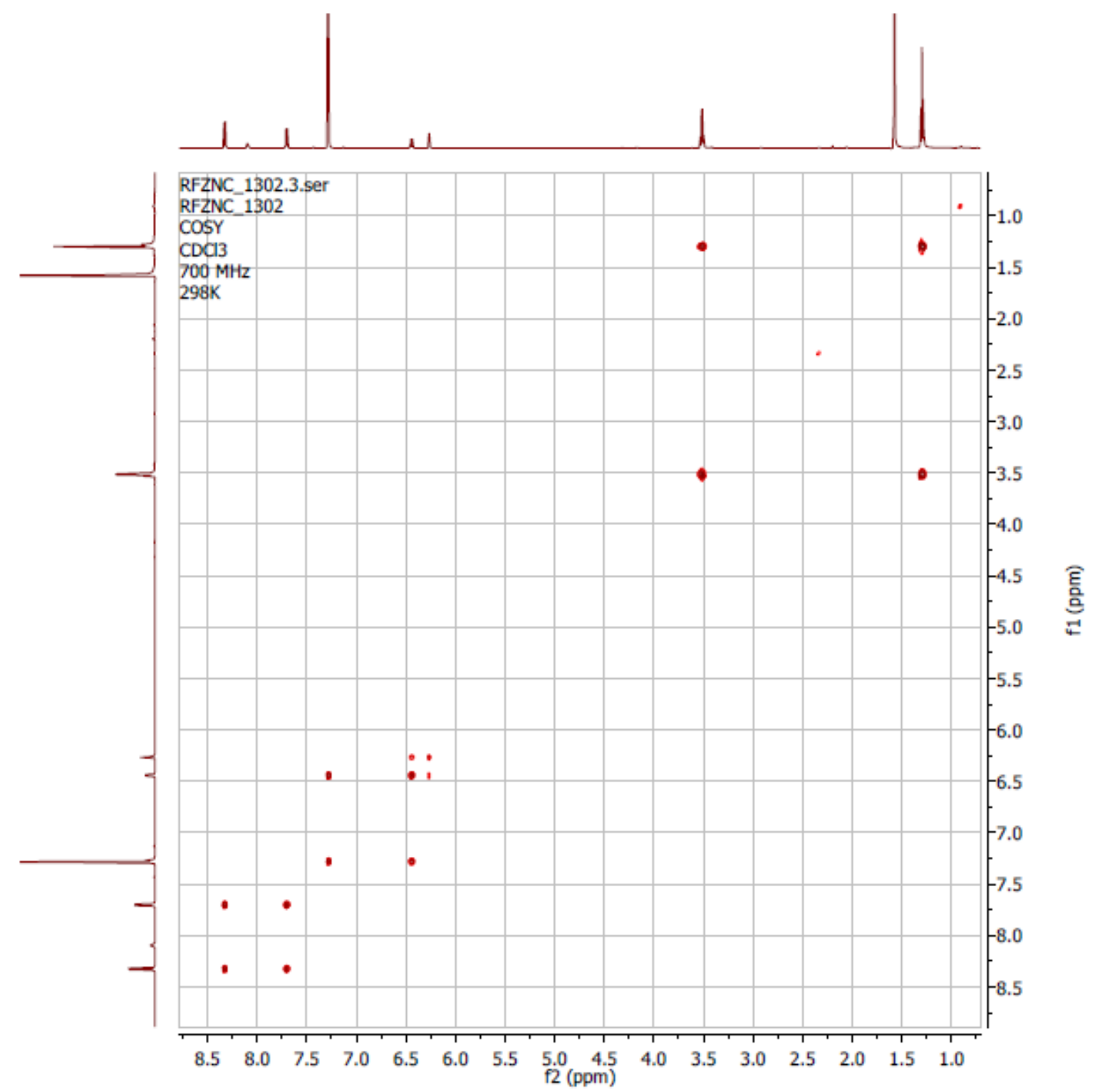

Figure S17. COSY-NMR (700 MHz) spectrum recorded for $\mathrm{B}\left(\mathrm{NO}_{2}\right)$ in $\mathrm{CDCl}_{3}$ at room temperature. 


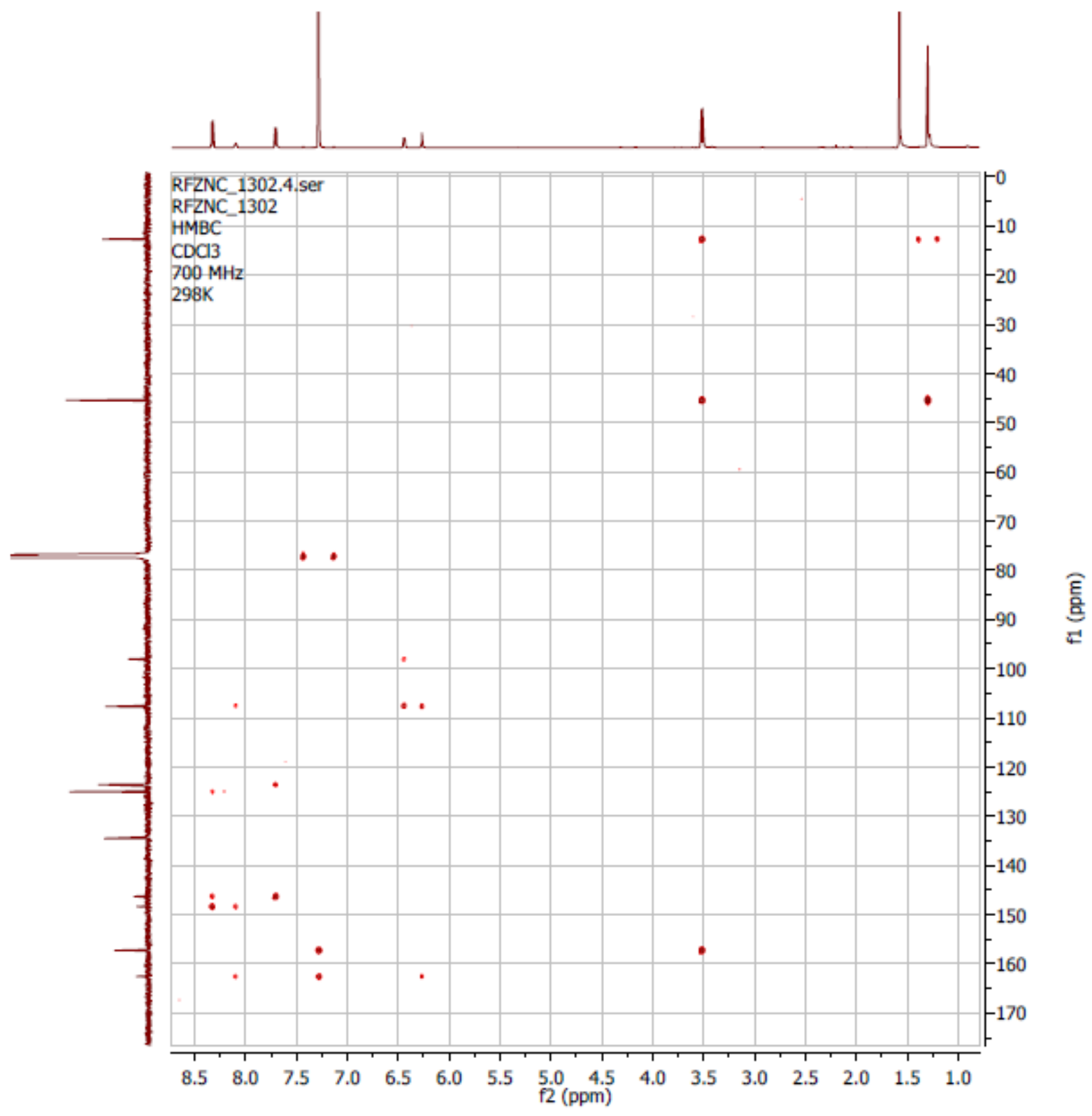

Figure S18. $\mathrm{HMBC}-\mathrm{NMR}(700 \mathrm{MHz})$ spectrum recorded for $\mathrm{B}\left(\mathrm{NO}_{2}\right)$ in $\mathrm{CDCl}_{3}$ at room temperature. 


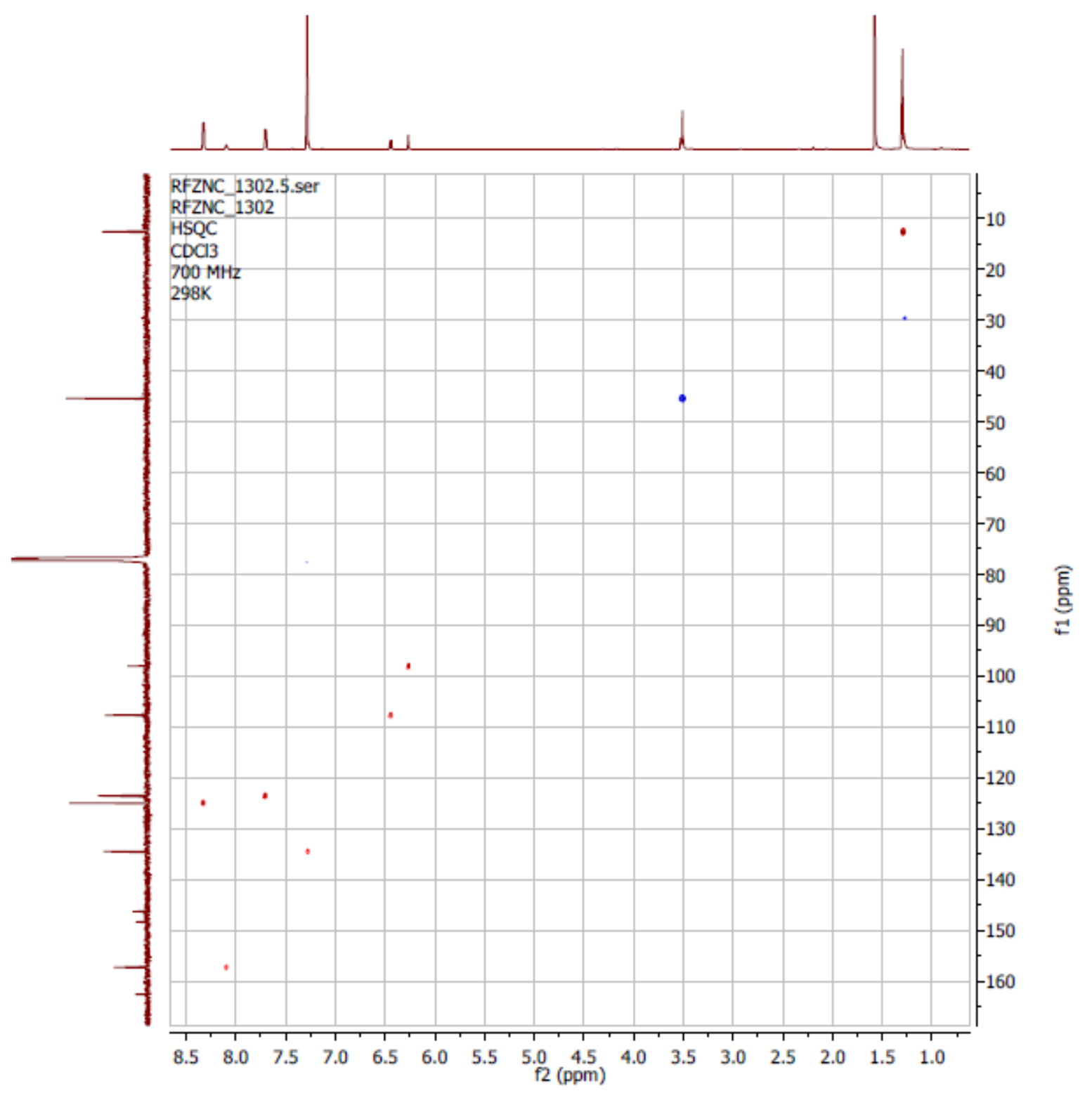

Figure S19. HSQC-NMR (700 MHz) spectrum recorded for $\mathrm{B}\left(\mathrm{NO}_{2}\right)$ in $\mathrm{CDCl}_{3}$ at room temperature. 


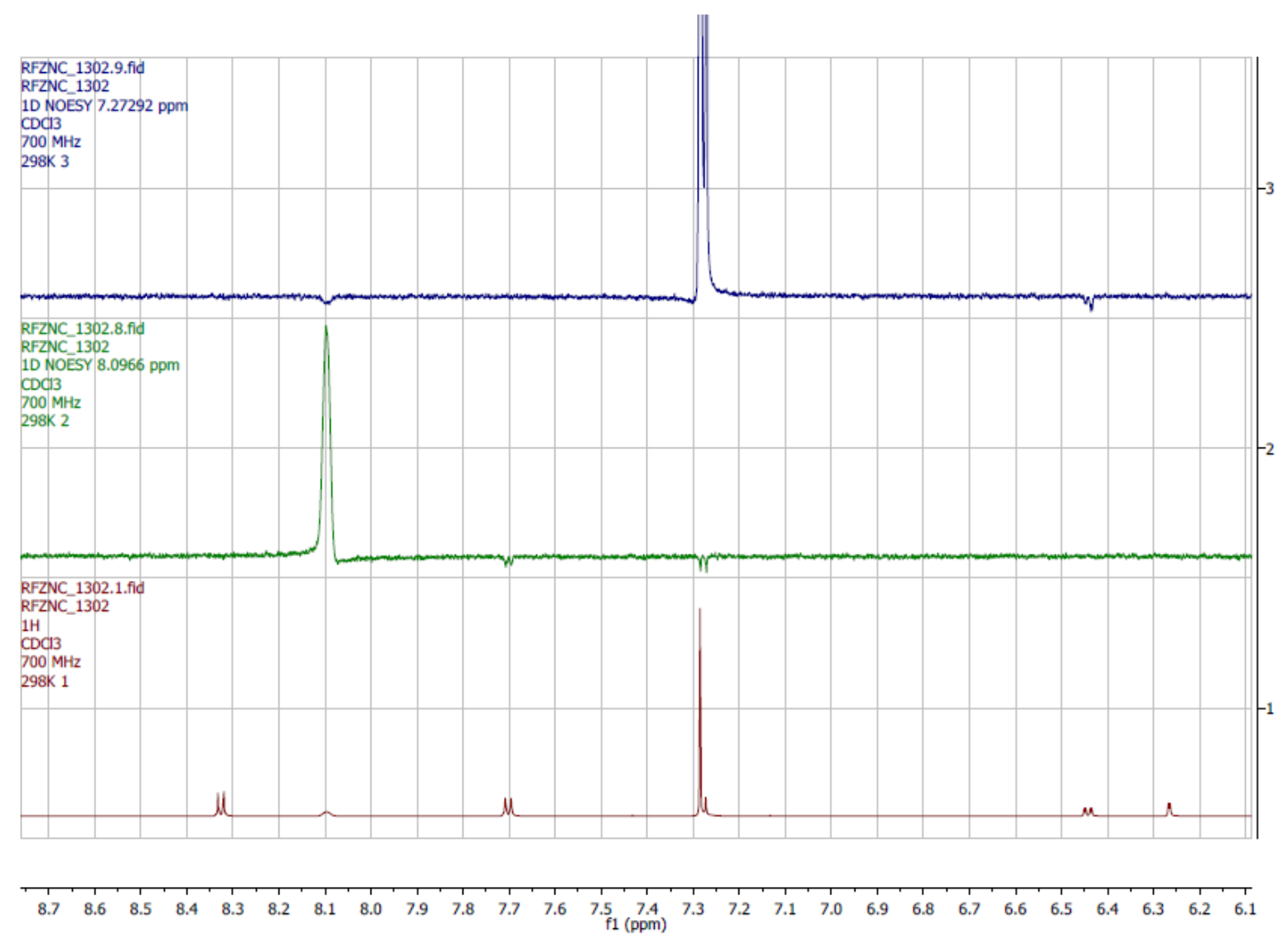

Figure S20. 1D-NOESY NMR (700 MHz) spectra recorded for $\mathrm{B}\left(\mathrm{NO}_{2}\right)$ in $\mathrm{CDCl}_{3}$ at room temperature. See Table S3 for assignments.

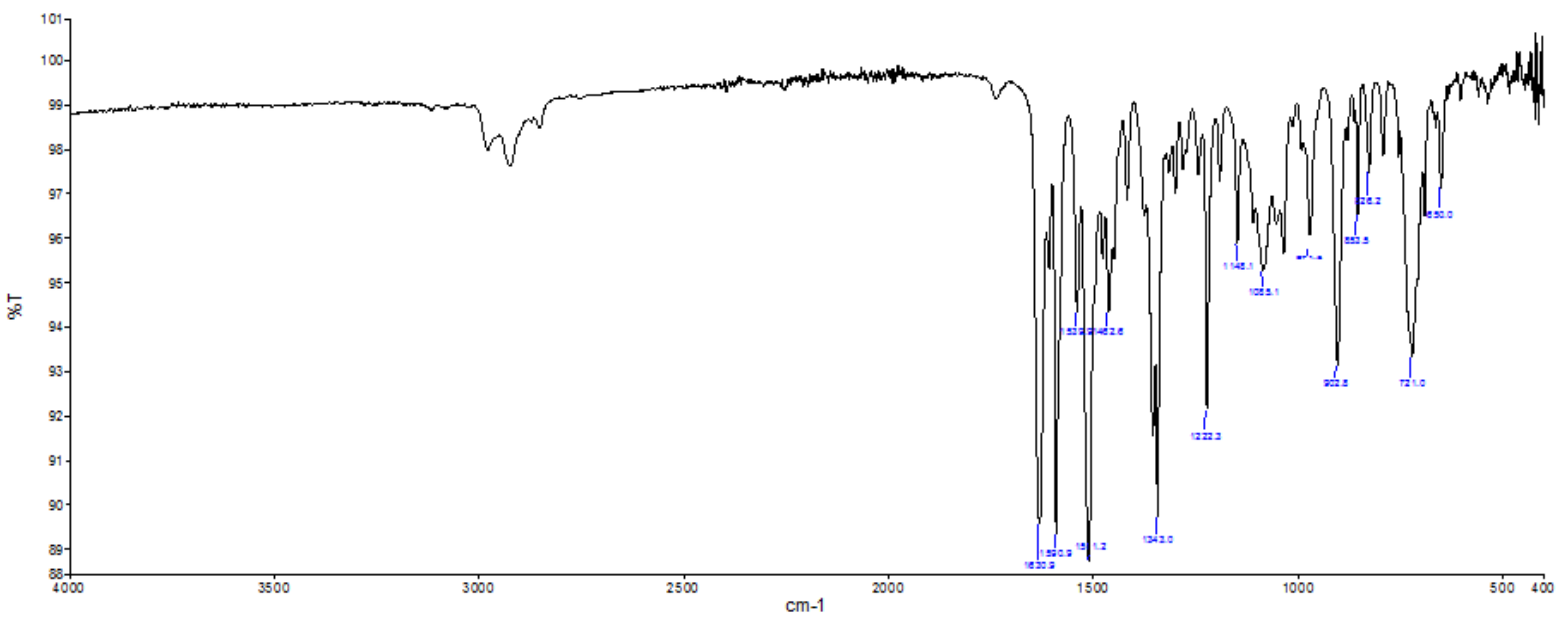

Figure S21. FT-IR spectrum recorded for $\mathrm{B}\left(\mathrm{NO}_{2}\right)$ in the solid state. 
Table S1. Summary of the ${ }^{1} \mathrm{H}$ and ${ }^{13} \mathrm{C}$ assignments made for $\mathrm{B}(\mathrm{H})$ and the atom numbering system.

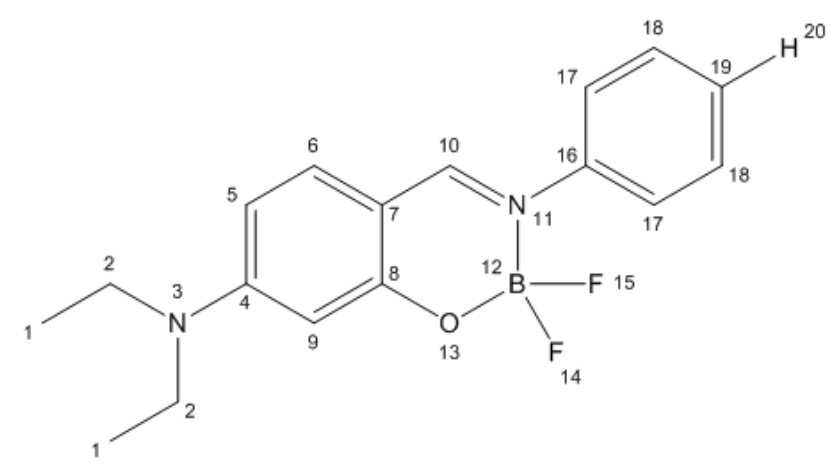

\begin{tabular}{|c|c|c|}
\hline \multirow[t]{2}{*}{ Nucleus Label } & \multicolumn{2}{|c|}{$\begin{array}{c}\text { Nucleus (chemical shift ppm, } \\
\text { splitting pattern) }\end{array}$} \\
\hline & ${ }^{1} \mathrm{H}$ & ${ }^{13} \mathrm{C}\left\{{ }^{1} \mathrm{H}\right\}$ \\
\hline 1 & $1.27, \mathrm{t}, \mathrm{J}_{\mathrm{HH}}=7.18 \mathrm{~Hz}$ & 12.7 \\
\hline 2 & $3.49, \mathrm{q}, \mathrm{J}_{\mathrm{HH}}=7.18 \mathrm{~Hz}$ & 45.2 \\
\hline 3 & - & - \\
\hline 4 & - & 156.2 \\
\hline 5 & $\begin{array}{l}6.39, \mathrm{dd}, \mathrm{J}_{\mathrm{HH}}=2.33 \mathrm{~Hz}, \\
\mathrm{~J}_{\mathrm{HH}}=9.03 \mathrm{~Hz}\end{array}$ & 106.6 \\
\hline 6 & $7.25, \mathrm{~d}, \mathrm{~J}_{\mathrm{HH}}=9.03 \mathrm{~Hz}$ & 133.8 \\
\hline 7 & - & 106.9 \\
\hline 8 & - & 161.9 \\
\hline 9 & $6.28, \mathrm{~d}, \mathrm{~J}_{\mathrm{HH}}=2.33 \mathrm{~Hz}$ & 98.2 \\
\hline 10 & $8.07, \mathrm{bs}$ & 158.4 \\
\hline 11 & - & - \\
\hline 12 & - & - \\
\hline 13 & - & - \\
\hline 14 & - & - \\
\hline 15 & - & - \\
\hline 16 & - & 143.3 \\
\hline 17 & $7.53, \mathrm{~d}, \mathrm{~J}_{\mathrm{HH}}=8.01 \mathrm{~Hz}$ & 123.3 \\
\hline 18 & $\begin{array}{l}7.46, \mathrm{dd}, \mathrm{J}_{\mathrm{HH}}=8.01 \mathrm{~Hz}, \\
\mathrm{~J}_{\mathrm{HH}}=8.01 \mathrm{~Hz}\end{array}$ & 129.4 \\
\hline 19 & - & 127.7 \\
\hline 20 & $\begin{array}{l}7.37, \mathrm{dd}, \mathrm{J}_{\mathrm{HH}}=7.37 \mathrm{~Hz}, \\
\mathrm{~J}_{\mathrm{HH}}=8.01 \mathrm{~Hz}\end{array}$ & - \\
\hline
\end{tabular}


Table S2. Summary of the ${ }^{1} \mathrm{H}$ and ${ }^{13} \mathrm{C}$ assignments made for $\mathrm{B}(\mathrm{I})$ and the atom numbering system.

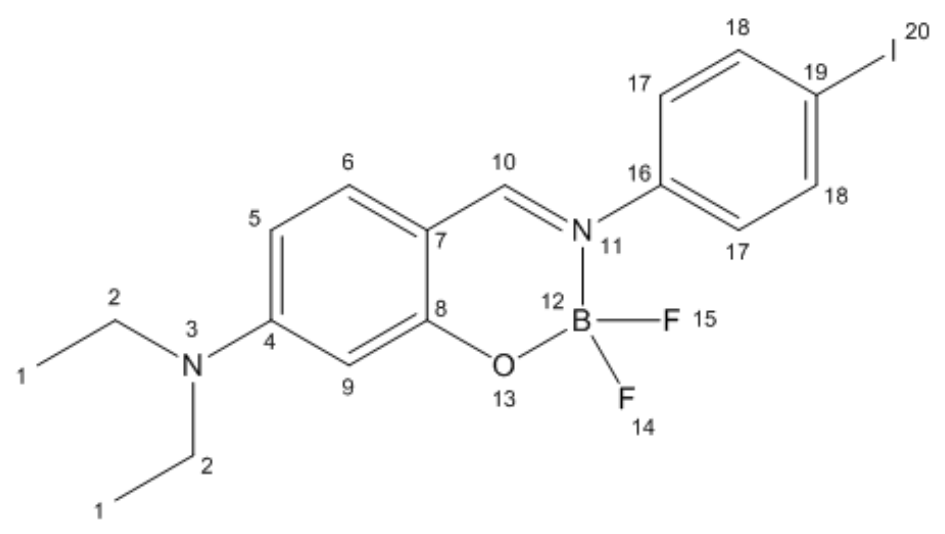

\begin{tabular}{|c|c|c|}
\hline \multirow[t]{2}{*}{ Nucleus Label } & \multicolumn{2}{|c|}{$\begin{array}{l}\text { Nucleus (chemical shift ppm, } \\
\text { splitting pattern) }\end{array}$} \\
\hline & ${ }^{1} \mathrm{H}$ & ${ }^{13} \mathrm{C}\left\{{ }^{1} \mathrm{H}\right\}$ \\
\hline 1 & $1.27, \mathrm{t}, \mathrm{J}_{\mathrm{HH}}=7.16 \mathrm{~Hz}$ & 12.8 \\
\hline 2 & $3.48, q, J_{\mathrm{HH}}=7.16 \mathrm{~Hz}$ & 45.3 \\
\hline 3 & - & - \\
\hline 4 & - & 156.5 \\
\hline 5 & $\begin{array}{l}6.39, \mathrm{dd}, \mathrm{J}_{\mathrm{HH}}=2.39 \mathrm{~Hz}, \\
\mathrm{~J}_{\mathrm{HH}}=9.07 \mathrm{~Hz}\end{array}$ & 106.9 \\
\hline 6 & $7.24, \mathrm{~d}, \mathrm{~J}_{\mathrm{HH}}=9.07 \mathrm{~Hz}$ & 134.0 \\
\hline 7 & - & 107.1 \\
\hline 8 & - & 162.0 \\
\hline 9 & $6.25, \mathrm{~d}, \mathrm{~J}_{\mathrm{HH}}=2.39 \mathrm{~Hz}$ & 98.1 \\
\hline 10 & $8.02, \mathrm{bs}$ & 157.7 \\
\hline 11 & - & - \\
\hline 12 & - & - \\
\hline 13 & - & - \\
\hline 14 & - & - \\
\hline 15 & - & - \\
\hline 16 & - & 142.9 \\
\hline 17 & $7.28, \mathrm{~d}, \mathrm{~J}_{\mathrm{HH}}=8.85 \mathrm{~Hz}$ & 125.0 \\
\hline 18 & $7.76, \mathrm{~d}, \mathrm{~J}_{\mathrm{HH}}=8.85 \mathrm{~Hz}$ & 138.5 \\
\hline 19 & - & 92.6 \\
\hline 20 & - & - \\
\hline
\end{tabular}


Table S3. Summary of the ${ }^{1} \mathrm{H}$ and ${ }^{13} \mathrm{C}$ assignments made for $\mathrm{B}\left(\mathrm{NO}_{2}\right)$ and the atom numbering system.

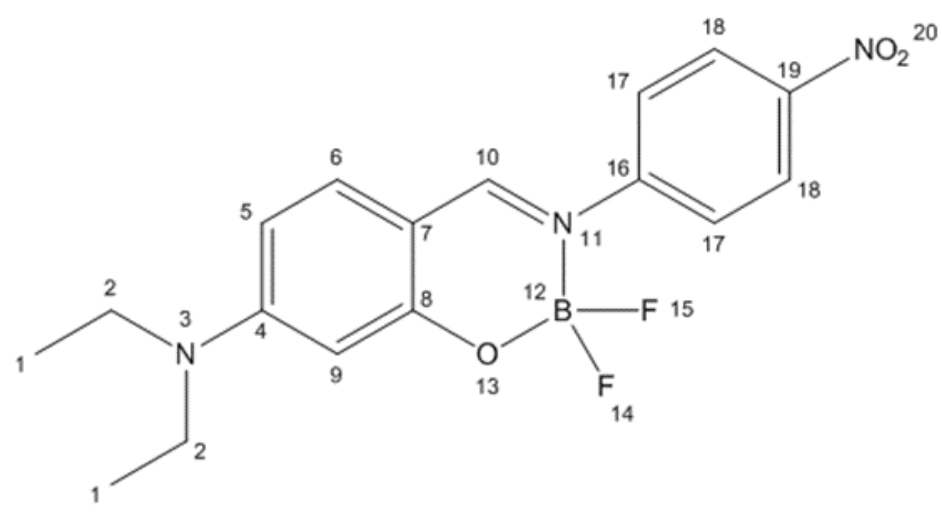

車

\begin{tabular}{|c|c|c|}
\hline \multirow[t]{2}{*}{ Nucleus Label } & \multicolumn{2}{|c|}{$\begin{array}{l}\text { Nucleus (chemical shift ppm, } \\
\text { splitting pattern) }\end{array}$} \\
\hline & ${ }^{1} \mathrm{H}$ & ${ }^{13} \mathrm{C}\left\{{ }^{1} \mathrm{H}\right\}$ \\
\hline 1 & $1.30, \mathrm{t}, \mathrm{J}_{\mathrm{HH}}=7.16 \mathrm{~Hz}$ & 12.70 \\
\hline 2 & $3.52, q, J_{H H}=7.16 \mathrm{~Hz}$ & 45.44 \\
\hline 3 & - & - \\
\hline 4 & - & 157.22 \\
\hline 5 & $\begin{array}{l}6.44, \mathrm{dd}, \mathrm{J}_{\mathrm{HH}}=2.37 \\
\mathrm{~Hz}, \mathrm{~J}_{\mathrm{HH}}=8.91 \mathrm{~Hz}\end{array}$ & 107.71 \\
\hline 6 & $7.27, \mathrm{~d}, \mathrm{~J}_{\mathrm{HH}}=8.91 \mathrm{~Hz}$ & 134.51 \\
\hline 7 & - & 107.53 \\
\hline 8 & - & 162.56 \\
\hline 9 & $6.27, \mathrm{~d}, \mathrm{~J}_{\mathrm{HH}}=2.37 \mathrm{~Hz}$ & 98.06 \\
\hline 10 & $8.10, \mathrm{bs}$ & 157.27 \\
\hline 11 & - & - \\
\hline 12 & - & - \\
\hline 13 & - & - \\
\hline 14 & - & - \\
\hline 15 & - & - \\
\hline 16 & - & 148.34 \\
\hline 17 & $7.70, \mathrm{~d}, \mathrm{~J}_{\mathrm{HH}}=9.04 \mathrm{~Hz}$ & 123.54 \\
\hline 18 & $8.33, \mathrm{~d}, \mathrm{~J}_{\mathrm{HH}}=9.04 \mathrm{~Hz}$ & 125.01 \\
\hline 19 & - & 146.29 \\
\hline 20 & - & - \\
\hline
\end{tabular}




\section{S2. Atom numbering system for X-ray crystallography}

Figure S22 shows the atom numbering system used to identify the individual atoms present in any given boranil. Also shown is a representation lacking the hydrogen atoms in order to better emphasize the molecular framework.

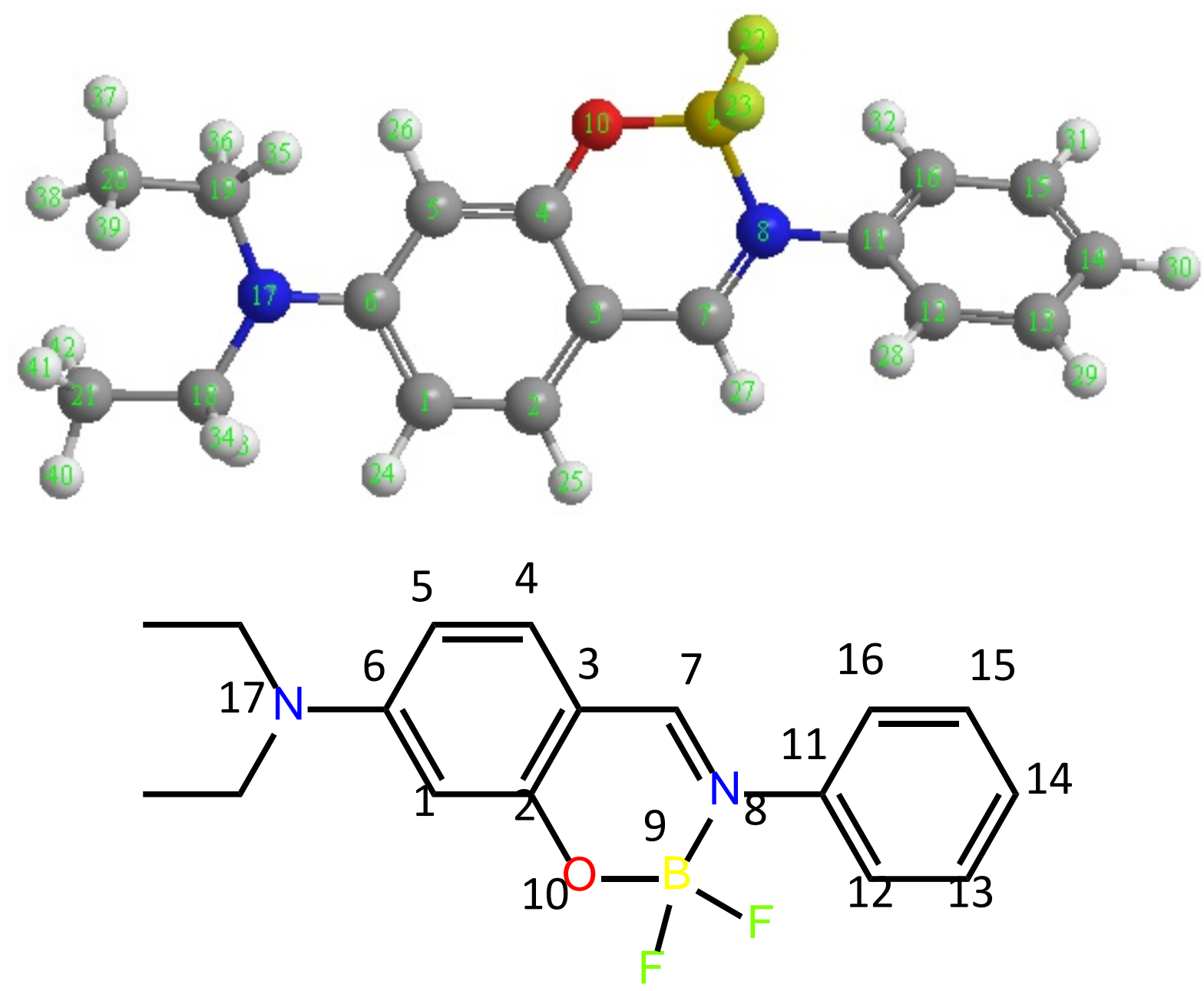

Figure S22. Full atom labelling system used throughout this work. Blue - nitrogen; red oxygen, yellow - boron, grey - carbon; white - hydrogen; lime - fluorine. The lower figure has the hydrogen atoms omitted for improved clarity. 


\section{S3. Structural details generated by computation}

Table S4. Cartesian coordinates computed for $\mathrm{B}(\mathrm{H})$ by DFT methods. ${ }^{(a)}$

\begin{tabular}{|c|c|c|c|c|}
\hline ATOM & C & $x$ & $\mathrm{Y}$ & Z \\
\hline C & 6.0 & -8.3950119325 & -2.9285160319 & 0.6664941756 \\
\hline C & 6.0 & -8.5466392365 & -0.5886977850 & 1.7094987477 \\
\hline C & 6.0 & -10.1335895116 & 1.2000921877 & 0.8648728738 \\
\hline C & 6.0 & -11.7237187166 & 0.6041220231 & -1.0185802789 \\
\hline C & 6.0 & -11.5806888805 & -1.7518583194 & -2.0172655798 \\
\hline C & 6.0 & -10.0172439076 & -3.6235141718 & -1.2454692278 \\
\hline C & 6.0 & -10.1522793208 & 3.5638300040 & 1.9164479457 \\
\hline$N$ & 7.0 & -11.4768933593 & 5.4615223359 & 1.2053469659 \\
\hline B & 5.0 & -13.9285561182 & 4.4624921657 & 0.2723351883 \\
\hline $\mathrm{O}$ & 8.0 & -13.2752997637 & 2.3164726665 & -1.8953817940 \\
\hline C & 6.0 & -11.6472618622 & 7.3668386436 & 2.6202284554 \\
\hline C & 6.0 & -11.6717819210 & 7.3347033975 & 5.2237608820 \\
\hline C & 6.0 & -11.8949001673 & 9.4336987207 & 6.6371882959 \\
\hline C & 6.0 & -11.9534828937 & 11.6692335137 & 5.4513105619 \\
\hline C & 6.0 & -11.8216264236 & 11.9105775964 & 2.9403558595 \\
\hline C & 6.0 & -11.7543669765 & 9.7128075243 & 1.5644045809 \\
\hline$N$ & 7.0 & -9.8560468215 & -5.8587213325 & -2.2418630499 \\
\hline C & 6.0 & -7.9936654880 & -7.5912322492 & -1.3359874252 \\
\hline C & 6.0 & -11.8196891380 & -6.3666241240 & -4.2109249596 \\
\hline C & 6.0 & -12.5124140588 & -9.0572208200 & -5.0936546459 \\
\hline C & 6.0 & -7.2473717366 & -10.1012161724 & -2.7926098284 \\
\hline $\mathrm{F}$ & 9.0 & -15.3994044442 & 6.5672824468 & -0.9694228810 \\
\hline $\mathrm{F}$ & 9.0 & -15.3366938378 & 3.3560036924 & 2.4593624106 \\
\hline $\mathrm{H}$ & 1.0 & -7.1171851585 & -4.1990782859 & 1.5701161832 \\
\hline $\mathrm{H}$ & 1.0 & -7.1142789774 & -0.1945920320 & 3.2660177424 \\
\hline $\mathrm{H}$ & 1.0 & -12.8850757962 & -2.0126634573 & -3.5682684768 \\
\hline $\mathrm{H}$ & 1.0 & -8.6791482308 & 3.8743806387 & 3.4842774752 \\
\hline $\mathrm{H}$ & 1.0 & -11.8054352963 & 5.3970680168 & 6.2324007240 \\
\hline $\mathrm{H}$ & 1.0 & -12.2079755350 & 9.3038140936 & 8.6098751756 \\
\hline $\mathrm{H}$ & 1.0 & -12.3529714114 & 13.4734097461 & 6.6501107766 \\
\hline $\mathrm{H}$ & 1.0 & -12.0375340192 & 13.5589858591 & 2.0172396104 \\
\hline $\mathrm{H}$ & 1.0 & -11.6420299985 & 9.9739131240 & -0.5223680859 \\
\hline $\mathrm{H}$ & 1.0 & -6.0940527361 & -6.6803218224 & -1.3723149014 \\
\hline $\mathrm{H}$ & 1.0 & -8.4885015797 & -8.2451964359 & 0.6322522565 \\
\hline $\mathrm{H}$ & 1.0 & -13.6625544939 & -5.8124212190 & -3.4485928311 \\
\hline $\mathrm{H}$ & 1.0 & -11.4274711698 & -5.2929222510 & -5.9430880519 \\
\hline 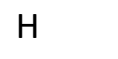 & 1.0 & -13.9570731261 & -9.2183923001 & -6.3899235879 \\
\hline $\mathrm{H}$ & 1.0 & -10.8489391771 & -9.9648599889 & -6.2717663914 \\
\hline & 1.0 & -12.8798615794 & -10.3502072949 & -3.5285593507 \\
\hline
\end{tabular}




$\begin{array}{lllll}H & 1.0 & -5.6464917898 & -11.0047299625 & -1.9419276503 \\ H & 1.0 & -8.7907470740 & -11.4549872127 & -2.6548416256 \\ H & 1.0 & -6.9115541412 & -9.6047005826 & -4.7976416891\end{array}$

(a) DFT/PBEO/6-311G+(s)/PCM/MT-correction

Table S5. Cartesian coordinates computed for $\mathrm{B}\left(\mathrm{NO}_{2}\right)$ by DFT methods. ${ }^{\text {(a) }}$

\begin{tabular}{|c|c|c|c|c|}
\hline ATOM & C & $\mathrm{X}$ & $Y$ & Z \\
\hline C & 6.0 & 1.3415338118 & -4.0943539546 & -1.7910435357 \\
\hline C & 6.0 & 2.9456257644 & -3.1093481471 & -0.0931338886 \\
\hline C & 6.0 & 2.6908870276 & -0.8431423657 & 0.9874230182 \\
\hline C & 6.0 & 0.7389869998 & 0.6704712824 & 0.3997897253 \\
\hline C & 6.0 & -0.9714627646 & -0.1996265107 & -1.2680995807 \\
\hline C & 6.0 & -0.6216186908 & -2.4970973185 & -2.2971175580 \\
\hline C & 6.0 & 0.4766125741 & 2.9364553076 & 1.4753522315 \\
\hline $\mathrm{N}$ & 7.0 & -1.1988304121 & 4.6711837983 & 0.9972536596 \\
\hline B & 5.0 & -3.6123462644 & 3.2806592543 & 0.3665348485 \\
\hline $\mathrm{O}$ & 8.0 & -3.0638361257 & 1.1933860675 & -1.8764000516 \\
\hline C & 6.0 & -1.5278063353 & 6.4976958942 & 2.5249544485 \\
\hline C & 6.0 & -1.3389252977 & 6.3090341221 & 5.0152012773 \\
\hline C & 6.0 & -1.7303018231 & 8.3671413056 & 6.5850199186 \\
\hline C & 6.0 & -2.3251220765 & 10.5827339305 & 5.6316051975 \\
\hline C & 6.0 & -2.5278817438 & 10.8283021551 & 3.0710919548 \\
\hline C & 6.0 & -2.1362564139 & 8.7507351577 & 1.5662595289 \\
\hline$N$ & 7.0 & 1.6026278307 & -6.2705910726 & -2.8623485709 \\
\hline C & 6.0 & 3.9757669677 & -7.6388618024 & -2.2317807835 \\
\hline C & 6.0 & 4.7775456668 & -9.9366071897 & -3.8279788891 \\
\hline C & 6.0 & -0.4747564973 & -7.2041733774 & -4.5220013646 \\
\hline C & 6.0 & -0.6602791161 & -9.9327929856 & -5.4514599024 \\
\hline $\mathrm{F}$ & 9.0 & -5.6734107477 & 5.1147989678 & -0.6487595015 \\
\hline $\mathrm{F}$ & 9.0 & -4.7207070863 & 1.9320028610 & 2.6833732711 \\
\hline$N$ & 7.0 & -2.6787309145 & 12.4033253885 & 7.0340780373 \\
\hline $\mathrm{O}$ & 8.0 & -3.3064447911 & 14.6303747072 & 6.1624982068 \\
\hline $\mathrm{O}$ & 8.0 & -2.5296141830 & 12.3005622376 & 9.4192843596 \\
\hline $\mathrm{H}$ & 1.0 & 4.6212429006 & -4.1121763689 & 0.5872354209 \\
\hline $\mathrm{H}$ & 1.0 & 4.0788467327 & -0.2220193738 & 2.3731228210 \\
\hline $\mathrm{H}$ & 1.0 & -2.1066887318 & -3.0006631821 & -3.6654027354 \\
\hline $\mathrm{H}$ & 1.0 & 1.9481269087 & 3.5258044290 & 2.8601083631 \\
\hline $\mathrm{H}$ & 1.0 & -0.9055889368 & 4.4750087968 & 6.0162358466 \\
\hline $\mathrm{H}$ & 1.0 & -1.5587369368 & 8.0528657767 & 8.6477460869 \\
\hline $\mathrm{H}$ & 1.0 & -3.0014975734 & 12.6417798849 & 2.1432787602 \\
\hline
\end{tabular}




$\begin{array}{lllll}\mathrm{H} & 1.0 & -2.3383661631 & 9.0650368195 & -0.4971134951 \\ \mathrm{H} & 1.0 & 5.6350043311 & -6.3985718667 & -2.4826919403 \\ \mathrm{H} & 1.0 & 3.8446280000 & -8.2755213587 & -0.2331667155 \\ \mathrm{H} & 1.0 & 6.7436672514 & -10.6785468111 & -3.2536645139 \\ \mathrm{H} & 1.0 & 3.5711480978 & -11.7555682015 & -3.5200369069 \\ \mathrm{H} & 1.0 & 4.8892808504 & -9.5550606924 & -5.8220825890 \\ \mathrm{H} & 1.0 & -2.3064951384 & -6.9002237879 & -3.5345233940 \\ \mathrm{H} & 1.0 & -0.4608883799 & -6.0150687566 & -6.2999289438 \\ \mathrm{H} & 1.0 & -2.4755064576 & -10.2724104236 & -6.5212319304 \\ \mathrm{H} & 1.0 & 0.8523442133 & -10.4976756618 & -6.7908985595 \\ \mathrm{H} & 1.0 & -0.6569106886 & -11.2508219045 & -3.8103982080\end{array}$

(a) DFT/PBE0/6-311G+(s)/PCM/MT-correction

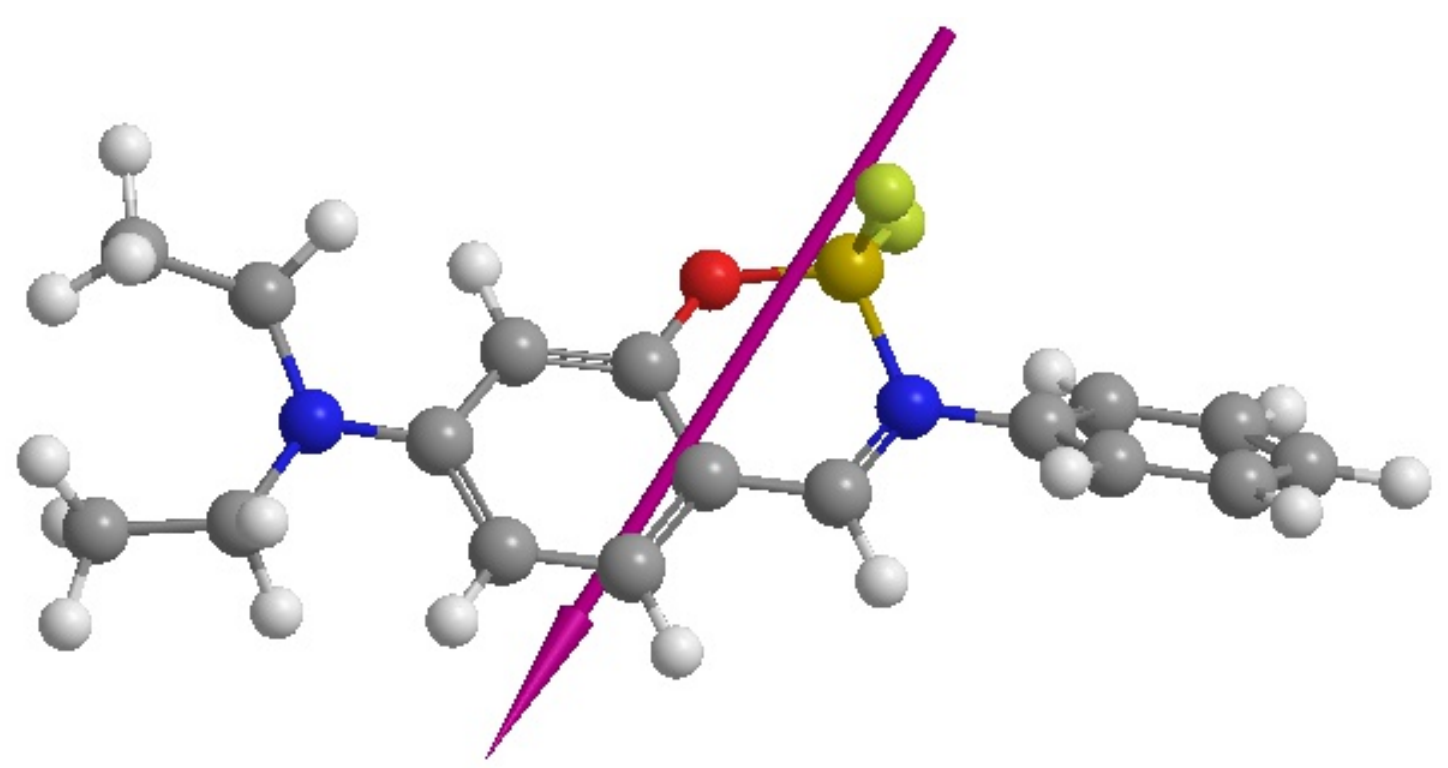

Figure S23. Indication of the direction of the permanent dipole moment vector computed ${ }^{\mathrm{S} 16}$ for $\mathrm{B}(\mathrm{H})$ in a reservoir of $\mathrm{CHCl}_{3}$ molecules. The solvent was modelled using the PCM method with the Mennucci-Tomasi correction. ${ }^{\mathrm{S} 13,14}$ The arrow superimposed on the energyminimized geometry indicates the direction of the dipole. Similar results were generated for $\mathrm{B}(\mathrm{I})$ and $\mathrm{B}\left(\mathrm{NO}_{2}\right)$. 

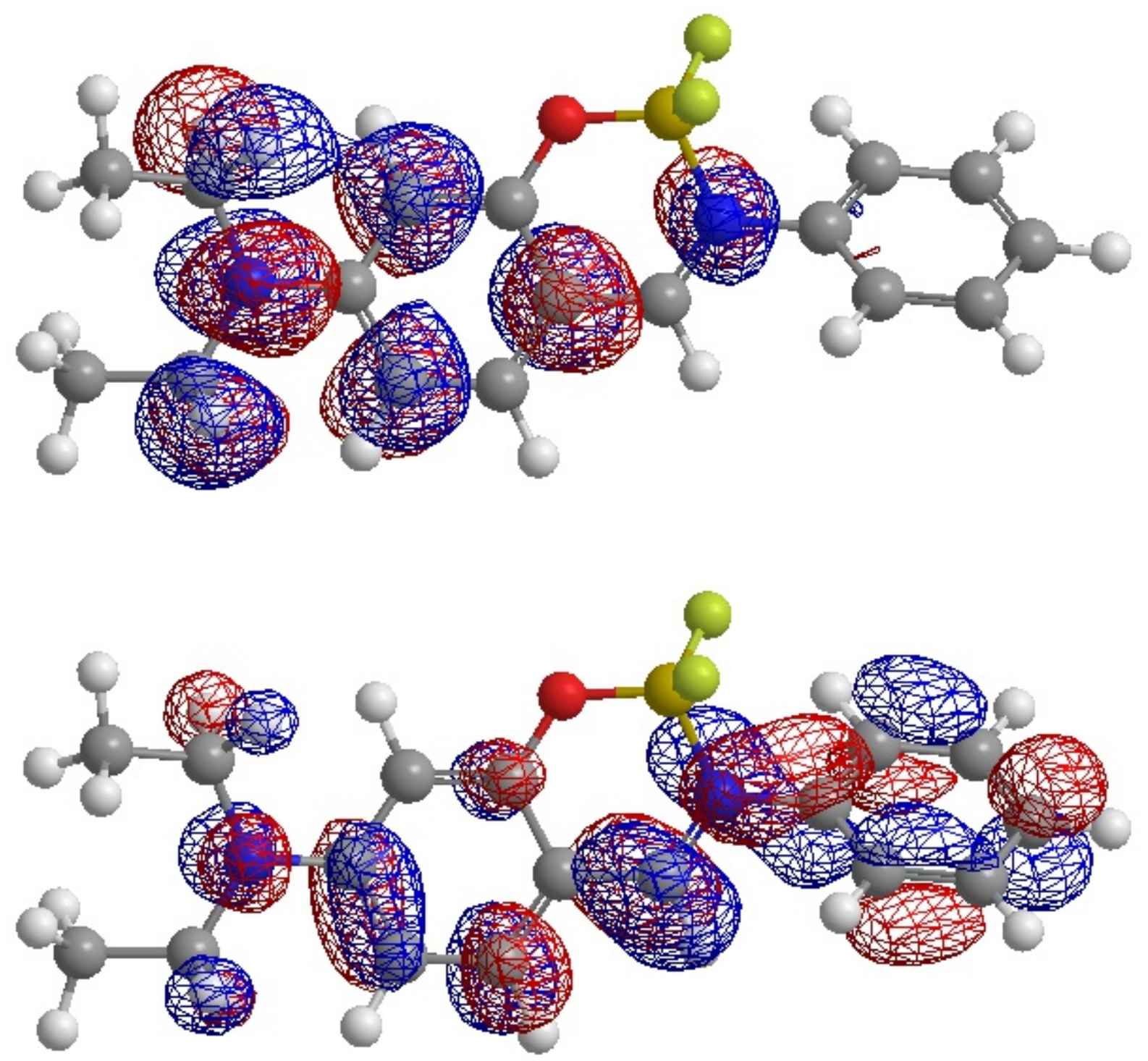

Figure S24. Kohn-Sham representations of the HOMO (upper panel) and LUMO (lower panel) computed for $\mathrm{B}(\mathrm{H})$ embedded in a reservoir of $\mathrm{CHCl}_{3}$ molecules. The calculations were made at the DFT/PBE0/6-311G+(s)/PCM level. 


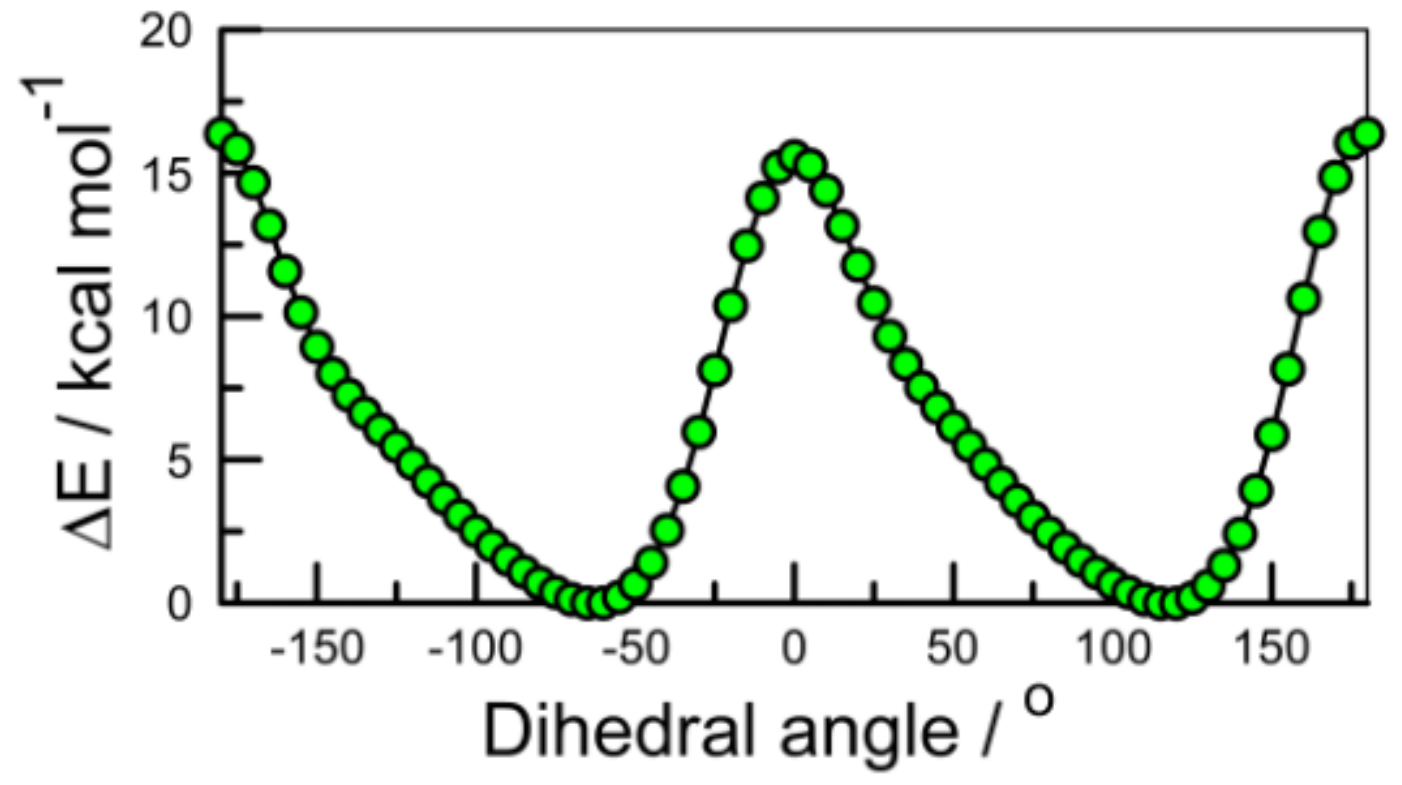

Figure S25. Computed rotational barrier for translation of the ancillary phenyl ring around the $\mathrm{N}_{8}-\mathrm{C}_{11}$ connection. The dihedral angle is shown for the $\mathrm{C}_{7}-\mathrm{N}_{8}-\mathrm{C}_{11}-\mathrm{C}_{12}$ fragment with the molecule embedded in a reservoir of $\mathrm{CHCl}_{3}$ molecules.

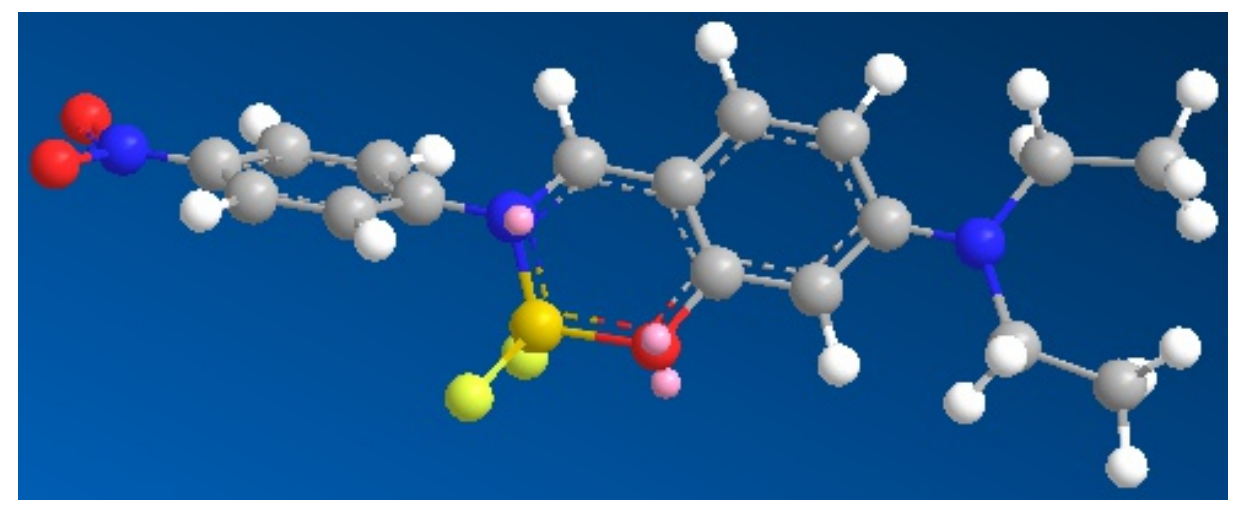

Figure S26. Energy-minimized geometry computed for $\mathrm{B}\left(\mathrm{NO}_{2}\right)$ in a reservoir of $\mathrm{CHCl}_{3}$ molecules (DFT/PBE0/6-311G(+d)/PCM). 


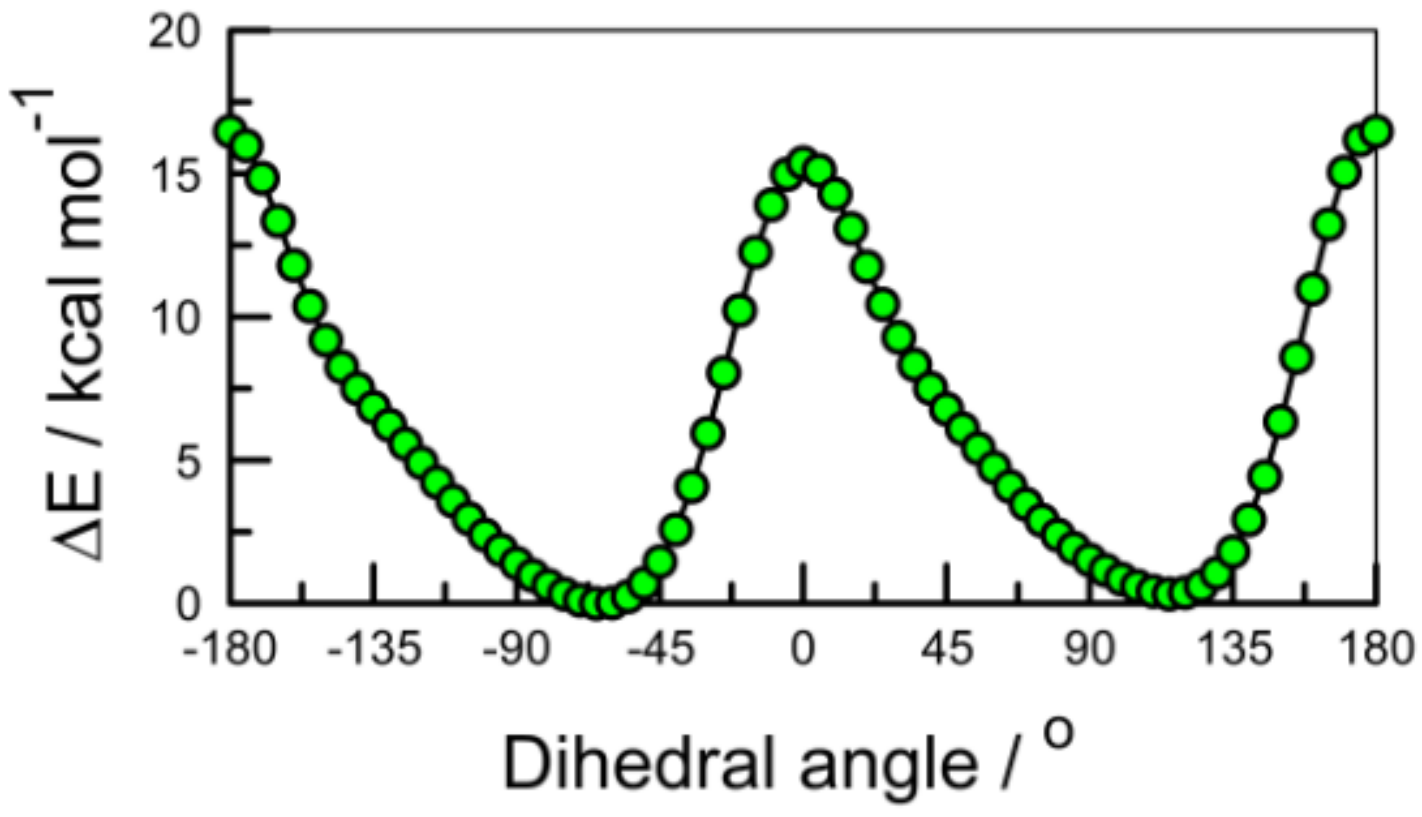

Figure S27. Rotational barrier calculation made for $\mathrm{B}\left(\mathrm{NO}_{2}\right)$ in a reservoir of $\mathrm{CHCl}_{3}$ molecules. Rotation was made around the $\mathrm{N}_{8}-\mathrm{C}_{11}$ bond while the dihedral angle refers to the $\mathrm{C}_{7}-\mathrm{N}_{8}-\mathrm{C}_{11^{-}}$ $C_{12}$ connection. 


\section{S4. Effect of solvent on the photophysical properties recorded in fluid solution.}

Table S6. Properties of the solvent taken from literature sources. ${ }^{\mathrm{S17}}$

\begin{tabular}{|c|c|c|c|c|c|c|c|c|}
\hline Solvent & $n^{\text {(a) }}$ & $\varepsilon s^{(b)}$ & $\eta / \mathrm{cP}^{\text {(c) }}$ & $P_{S O L}{ }^{(d)}$ & $F_{N}(e)$ & $E_{T}(30)^{(f)}$ & $Z^{\text {(g) }}$ & $\mathrm{SPP}^{(\mathrm{h})}$ \\
\hline $\mathrm{C}_{6} \mathrm{H}_{12}$ & 1.4263 & 2.02 & 0.98 & 0.204 & 0.101 & 31.2 & & 0.557 \\
\hline Toluene & 1.4969 & 2.38 & 0.587 & 0.226 & 0.126 & 33.2 & & 0.655 \\
\hline Anisole & 1.5170 & 4.33 & 1.15 & 0.232 & 0.229 & 37.2 & & \\
\hline $\mathrm{DMF}^{(\mathrm{i})}$ & 1.4305 & 36.71 & 0.924 & 0.206 & 0.377 & 43.8 & 68.5 & 0.954 \\
\hline $\mathrm{CH}_{3} \mathrm{CN}$ & 1.3441 & 37.5 & 0.345 & 0.175 & 0.375 & 46.0 & 71.3 & 0.895 \\
\hline THF (j) & 1.4072 & 7.58 & 0.55 & 0.198 & 0.308 & 37.4 & & 0.838 \\
\hline $\mathrm{CHCl}_{3}$ & 1.4459 & 4.81 & 0.58 & 0.222 & 0.248 & 39.1 & 63.2 & 0.786 \\
\hline Dioxane $^{(k)}$ & 1.4224 & 2.21 & 1.439 & 0.203 & 0.122 & 36.0 & & 0.701 \\
\hline $\mathrm{CH}_{2} \mathrm{Cl}_{2}$ & 1.4242 & 8.93 & 0.449 & 0.16 & 0.336 & 41.1 & 64.2 & 0.876 \\
\hline Acetone & 1.3587 & 20.7 & 0.304 & 0.180 & 0.375 & 42.2 & 65.7 & 0.881 \\
\hline Ether (I) & 1.3524 & 4.34 & 0.242 & 0.178 & 0.260 & 34.6 & & 0.694 \\
\hline
\end{tabular}

(a) Refractive index. (b) Static dielectric constant. (c) Shear viscosity. (d) Polarizability function from Equation S1. (e) Pekar function from Equation S2. (f) Dimroth's parameter derived from the absorption spectrum of pyridinium betaine. (g) Kosower's parameter derived from the absorption spectrum of 1-ethyl-4-methoxycarbonylpyridinium iodide. (h) Catalan's SPP polarizability function. (i) N,N-Dimethylformamide. (j) Tetrahydrofuran. (k) 1,4-Dioxane. (I) Diethyl ether.

$$
\begin{gathered}
P_{S O L}=\frac{n^{2}-1}{2 n^{2}+1} \\
F_{N}=\frac{\varepsilon_{S}-1}{2 \varepsilon_{S}+1}-\frac{P_{S O L}}{2}
\end{gathered}
$$


Table S7. Photophysical properties determined for $\mathrm{B}(\mathrm{H})$ in fluid solution at $20^{\circ} \mathrm{C}$.

\begin{tabular}{|l|l|l|l|l|l|l|}
\hline Solvent & $\lambda_{\mathrm{ABS}}{ }^{(\mathrm{a})}$ & $\lambda_{\mathrm{FLU}}{ }^{(\mathrm{b})}$ & $\Phi_{\mathrm{F}}{ }^{(\mathrm{c})}$ & $\tau / \mathrm{ns}$ & $\mathrm{k}_{\mathrm{RAD}} / 10^{8} \mathrm{~s}^{-1}(\mathrm{e})$ & $\mathrm{k}_{\mathrm{NR}} / 10^{8} \mathrm{~s}^{-1(\mathrm{f})}$ \\
\hline $\mathrm{C}_{6} \mathrm{H}_{12}$ & 392.5 & 446 & 0.63 & 1.75 & 0.36 & 0.21 \\
\hline Toluene & 397.5 & 452.5 & 0.58 & 1.72 & 0.34 & 0.24 \\
\hline Anisole & 400 & 456.5 & 0.155 & 1.70 & 0.091 & 0.50 \\
\hline DMF & 398 & 459 & 0.0045 & 0.095 & 0.047 & 21 \\
\hline $\mathrm{CH}_{3} \mathrm{CN}$ & 395 & 458 & 0.0065 & 0.075 & 0.087 & 11 \\
\hline $\mathrm{THF}$ & 394.5 & 455 & 0.125 & 0.55 & 0.23 & 1.6 \\
\hline $\mathrm{CHCl}_{3}$ & 394 & 456 & 0.108 & 0.52 & 0.21 & 1.7 \\
\hline $1,4-$ Dioxane & 393 & 453 & 0.120 & 0.78 & 0.15 & 1.1 \\
\hline $\mathrm{CH}_{2} \mathrm{Cl}_{2}$ & 395 & 457 & 0.098 & 0.47 & 0.21 & 4.3 \\
\hline Acetone & 394 & 456 & 0.020 & 0.16 & 0.13 & 7.5 \\
\hline Ether & 393 & 453 & 0.26 & 1.07 & 0.24 & 3.1 \\
\hline
\end{tabular}

(a) Absorption maximum, $\pm 0.5 \mathrm{~nm}$. (b) Fluorescence maximum, $\pm 1 \mathrm{~nm}$. (c) Fluorescence quantum yield measured relative to a standard compound, $\pm 8 \%$. (d) Fluorescence lifetime determined by time-correlated, single photon counting, $\pm 10 \%$. (e) Radiative rate constant. (f) Radiationless rate constant. 
Table S8. Photophysical properties determined for $\mathrm{B}(\mathrm{I})$ in fluid solution at $20^{\circ} \mathrm{C}$.

\begin{tabular}{|l|l|l|l|l|l|l|}
\hline Solvent & $\lambda_{\mathrm{ABS}}{ }^{(\mathrm{a})}$ & $\lambda_{\mathrm{FLU}}{ }^{(\mathrm{b})}$ & $\Phi_{\mathrm{F}}(\mathrm{c})$ & $\tau / \mathrm{ns}{ }^{(\mathrm{d})}$ & $\mathrm{k}_{\mathrm{RAD}} / 10^{8} \mathrm{~s}^{-1}(\mathrm{e})$ & $\mathrm{k}_{\mathrm{NR}} / 10^{8} \mathrm{~s}^{-1(\mathrm{f})}$ \\
\hline $\mathrm{C}_{6} \mathrm{H}_{12}$ & 401 & 457 & 0.22 & 0.77 & 0.29 & 1.0 \\
\hline Toluene & $\begin{array}{l}405 \\
(403)^{(\mathrm{g})}\end{array}$ & $\begin{array}{l}460 \\
(455)\end{array}$ & $\begin{array}{l}0.17 \\
(0.09)\end{array}$ & $\begin{array}{l}0.72 \\
(0.27)\end{array}$ & $\begin{array}{l}0.24 \\
(0.33)\end{array}$ & $\begin{array}{l}1.2 \\
(3.4)\end{array}$ \\
\hline Anisole & 408.5 & 466 & 0.16 & 0.65 & 0.25 & 1.3 \\
\hline DMF & 406 & 467 & 0.0090 & 0.095 & 0.095 & 10 \\
\hline $\mathrm{CH}_{3} \mathrm{CN}$ & 404 & 465 & 0.0055 & 0.075 & 0.073 & 13 \\
\hline $\mathrm{THF}^{\mathrm{CHCl}} 3$ & 404 & 463 & 0.104 & 0.48 & 0.22 & 1.9 \\
\hline $1,4-$ Dioxane & 401 & 460 & 0.12 & 0.44 & 0.27 & 2.4 \\
\hline $\mathrm{CH}_{2} \mathrm{Cl}{ }_{2}$ & 406 & 465 & 0.077 & 0.45 & 0.17 & 2.1 \\
\hline Acetone & 407 & 464 & 0.022 & 0.16 & 0.14 & 6.1 \\
\hline Ether & 404 & 462 & 0.140 & 0.61 & 0.23 & 1.4 \\
\hline
\end{tabular}

(a) Absorption maximum, $\pm 0.5 \mathrm{~nm}$. (b) Fluorescence maximum, $\pm 1 \mathrm{~nm}$. (c) Fluorescence quantum yield measured relative to a standard compound, $\pm 8 \%$. (d) Fluorescence lifetime determined by time-correlated, single photon counting, $\pm 10 \%$. (e) Radiative rate constant. (f) Radiationless rate constant. (g) Literature value from Ref S1. 
Table S9. Photophysical properties determined for $\mathrm{B}\left(\mathrm{NO}_{2}\right)$ in fluid solution at $20^{\circ} \mathrm{C}$.

\begin{tabular}{|l|l|l|l|l|l|l|}
\hline Solvent & $\lambda_{\mathrm{ABS}}{ }^{(\mathrm{a})}$ & $\lambda_{\mathrm{FLU}}{ }^{(\mathrm{b})}$ & $\Phi_{\mathrm{F}}{ }^{(\mathrm{c})}$ & $\tau / \mathrm{ns}^{(\mathrm{d})}$ & $\mathrm{k}_{\mathrm{RAD}} / 10^{8} \mathrm{~s}^{-1}(\mathrm{e})$ & $\mathrm{k}_{\mathrm{NR}} / 10^{8} \mathrm{~s}^{-1(\mathrm{f})}$ \\
\hline $\mathrm{C}_{6} \mathrm{H}_{12}$ & 420 & 469 & 0.58 & 1.75 & 3.3 & 2.4 \\
\hline Toluene & 427 & 478 & 0.50 & 1.61 & 3.1 & 3.1 \\
& $427^{(\mathrm{g})}$ & 474 & 0.60 & 1.61 & 3.7 & 2.5 \\
\hline Anisole & 432.5 & 490 & 0.54 & 1.78 & 3.0 & 2.6 \\
\hline DMF & & & 0.0097 & 0.080 & 1.2 & 12.4 \\
\hline $\mathrm{CH}_{3} \mathrm{CN}$ & 427 & 500 & 0.0090 & $<0.060$ & $\mathrm{NA}$ & \\
& 429 & 496 & $<0.01$ & $\mathrm{NA}$ & & \\
\hline $\mathrm{THF}$ & 429 & 494 & 0.14 & 0.61 & 2.3 & 1.4 \\
\hline $\mathrm{CHCl}_{3}$ & $429^{(\mathrm{h})}$ & 507 & 0.13 & 0.78 & 1.7 & 1.1 \\
\hline $1,4-$ Dioxane & 423.5 & 495 & 0.13 & 0.58 & 2.2 & 1.5 \\
\hline $\mathrm{CH}_{2} \mathrm{Cl}_{2}$ & 424 & 497 & 0.41 & 1.29 & 3.2 & 8.5 \\
\hline Acetone & 425 & 481 & 0.019 & 0.095 & 2.0 & 4.6 \\
\hline Ether & 424 & 495 & 0.056 & 0.21 & 2.7 & 10.3 \\
& $422^{(\mathrm{h})}$ & & 0.41 & 1.29 & 3.2 & 4.5 \\
\hline
\end{tabular}

(a) Absorption maximum. (b) Fluorescence maximum. (c) Fluorescence quantum yield measured relative to a standard compound. (d) Fluorescence lifetime determined by timecorrelated, single photon counting. (e) Radiative rate constant. (f) Radiationless rate constant. (g) Literature value from Ref. S1. (h) Literature value from Ref. S18. 


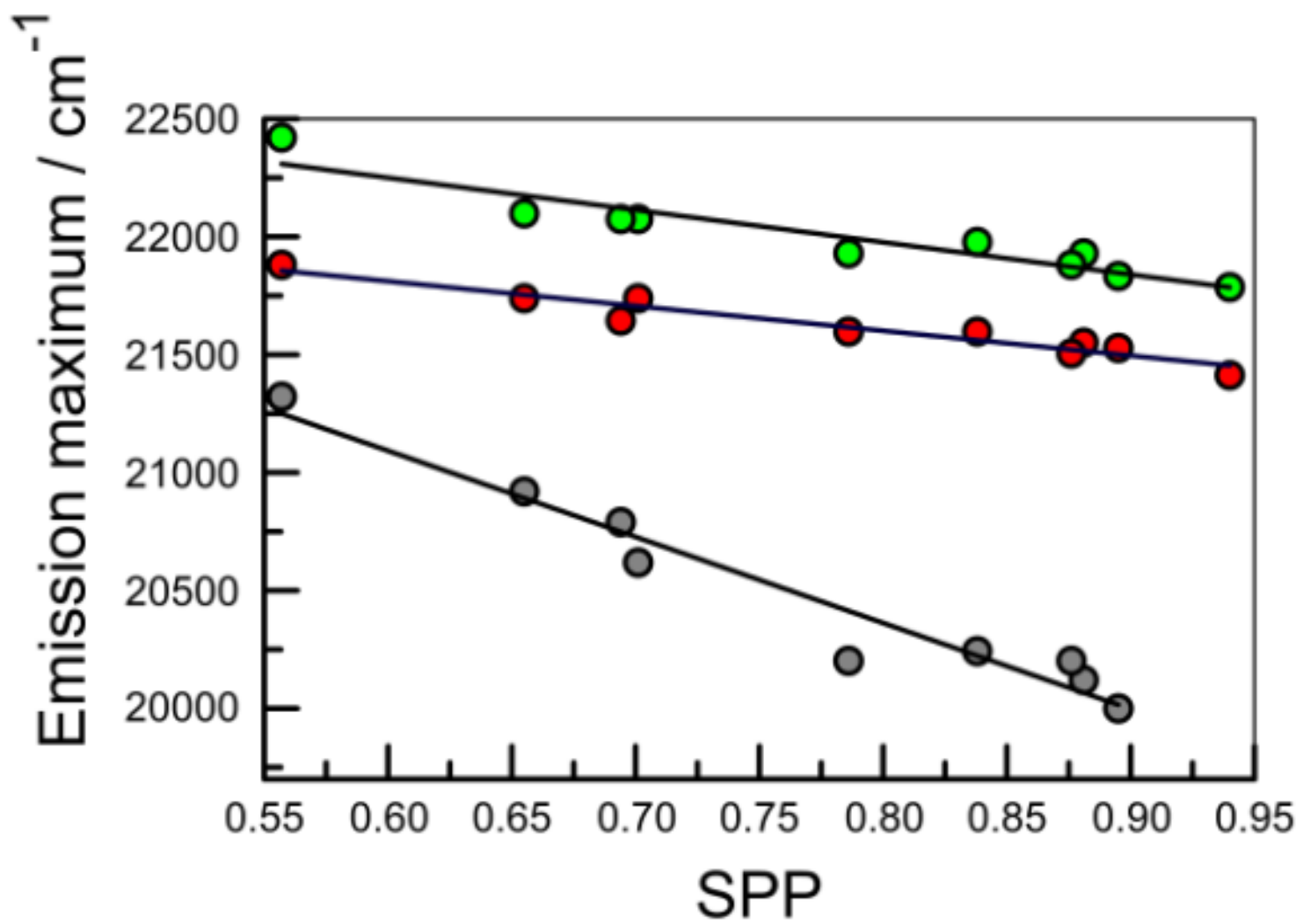

Figure S28. Correlation between the emission maximum in $\mathrm{cm}^{-1}$ and the empirical Catalán solvent polarizability function (SPP). The experimental data refer to $B(H)$ (green points), $B(I)$ (red points) and $\mathrm{B}\left(\mathrm{NO}_{2}\right)$ (grey points. The solid line drawn through each set of points is a fit to Equation S3, for which the derived parameters are provided in Table S7.

$$
v_{F L U}=v_{0}-\beta \times S P P
$$

Table S7. Parameters associated with a linear fit to Equation S3.

\begin{tabular}{|c|c|c|c|c|}
\hline Boranil & $v_{0} / \mathrm{cm}^{-1}$ & $\beta$ & $\begin{array}{c}\text { Correlation } \\
\text { coefficient }\end{array}$ & Dataset \\
\hline $\mathrm{B}(\mathrm{H})$ & 23,070 & 1,367 & 0.942 & 11 \\
\hline $\mathrm{B}(\mathrm{l})$ & 22,440 & 1,051 & 0.963 & 11 \\
\hline $\mathrm{B}\left(\mathrm{NO}_{2}\right)$ & 23,285 & 3,365 & 0.974 & 10 \\
\hline
\end{tabular}




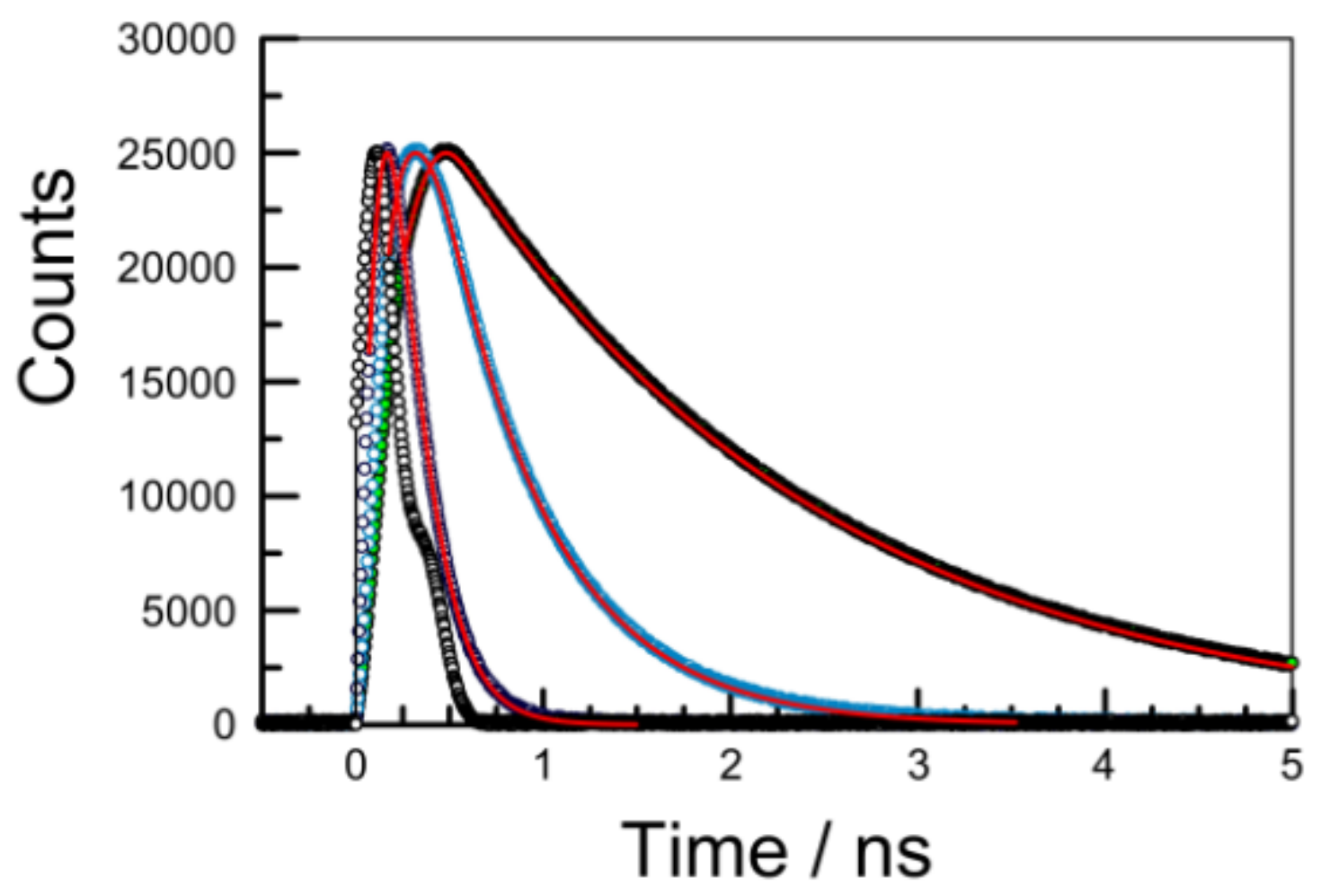

Figure S29. Examples of time-resolved fluorescence decay curves recorded for $B(H)$ in fluid solution at room temperature. Excitation was made at $370 \mathrm{~nm}$ and emission was detected at $450 \pm 10 \mathrm{~nm}$. The instrumental response function is shown as black circles. Individual decay curves refer to cyclohexane (black/green, $\chi^{2}=1.06$ ), tetrahydrofuran (cyan, $\chi^{2}=1.04$ ) and acetone blue, $\left.\chi^{2}=1.09\right)$. In each case, the fit to a mono-exponential decay is superimposed over the data points as a red line. 


\section{S5. Emission from the solid-state.}

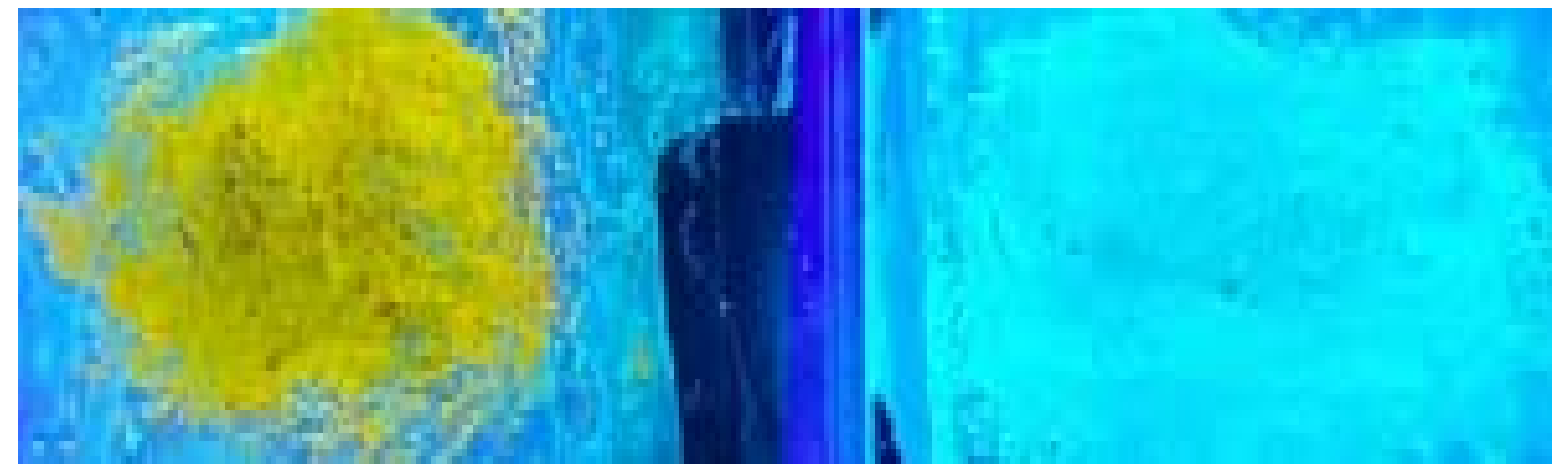

Figure S30. Photographs showing crystals of $B(I)$ in ambient room lighting (left-hand side) and under near-UV excitation (right-hand side). The crystals were simply dispersed on a quartz microscope slide and sealed with a layer of polyethylene.

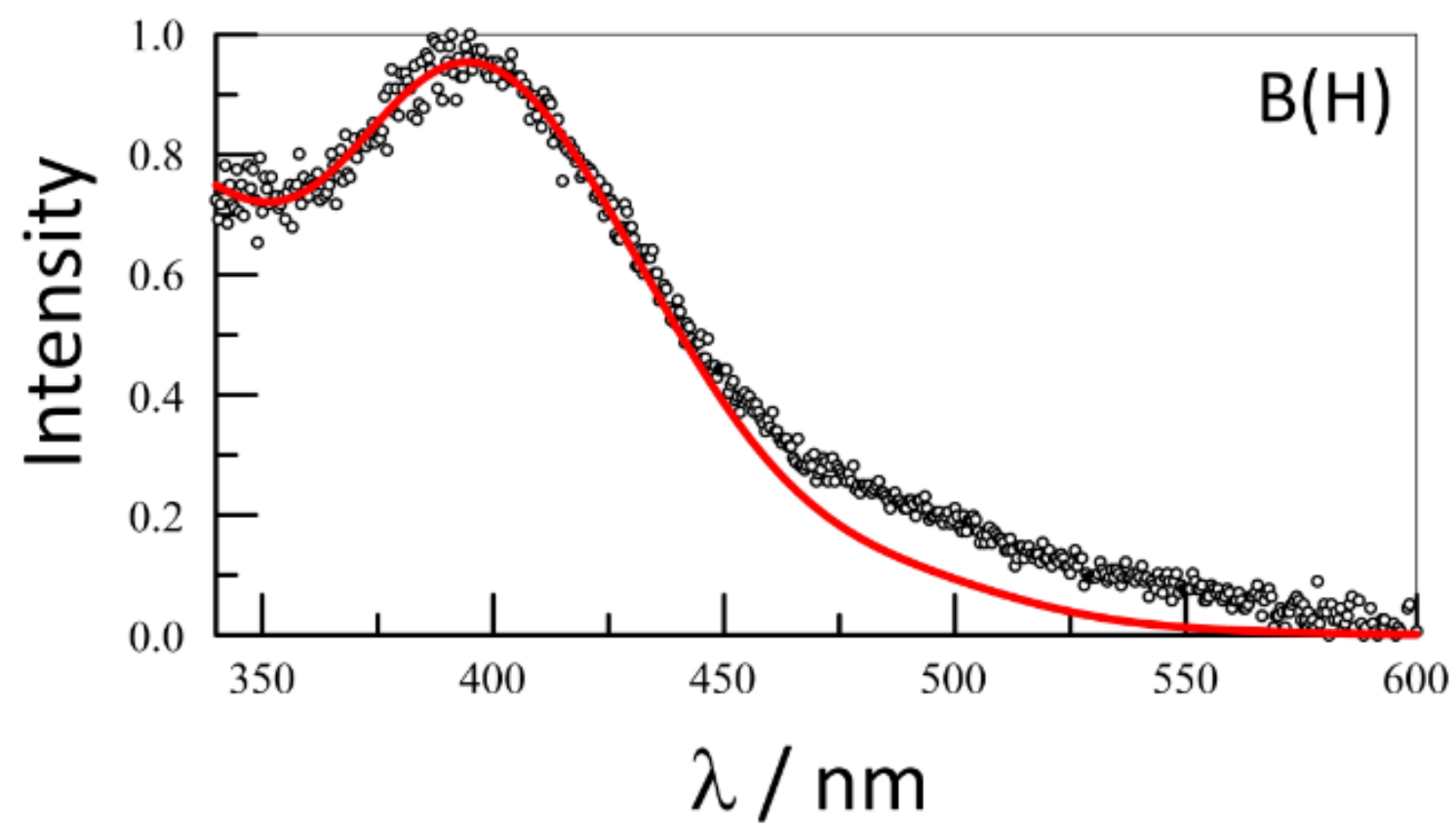

Figure S31. Comparison of normalized absorption (black circles) and excitation (red curve) spectra recorded for crystals of $\mathrm{B}(\mathrm{H})$ at room temperature. The emission wavelength was 550 $\mathrm{nm}$. 


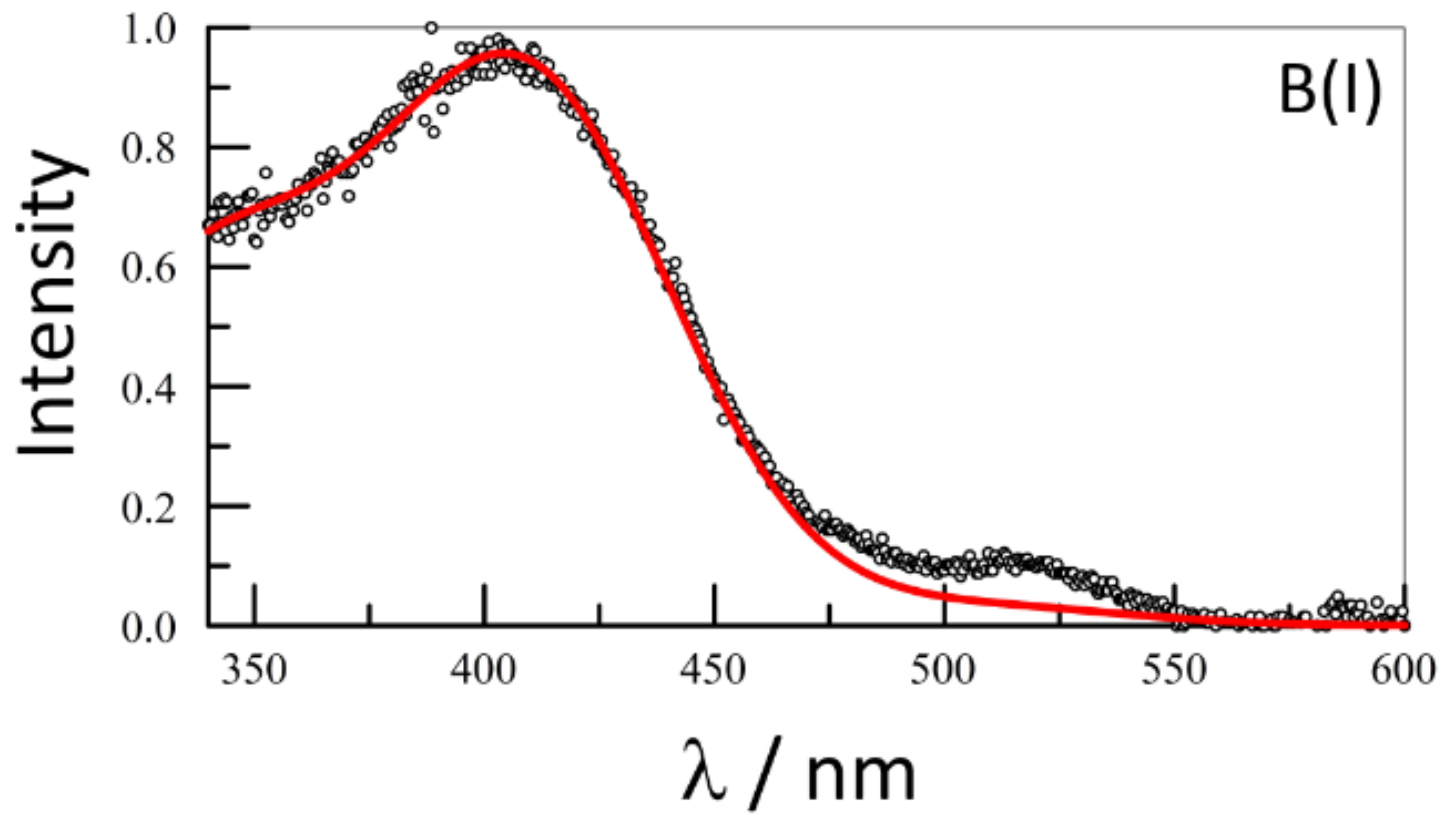

Figure S32. Comparison of normalized absorption (black circles) and excitation (red curve) spectra recorded for crystals of $\mathrm{B}(\mathrm{I})$ at room temperature. The emission wavelength was 525 $\mathrm{nm}$. 


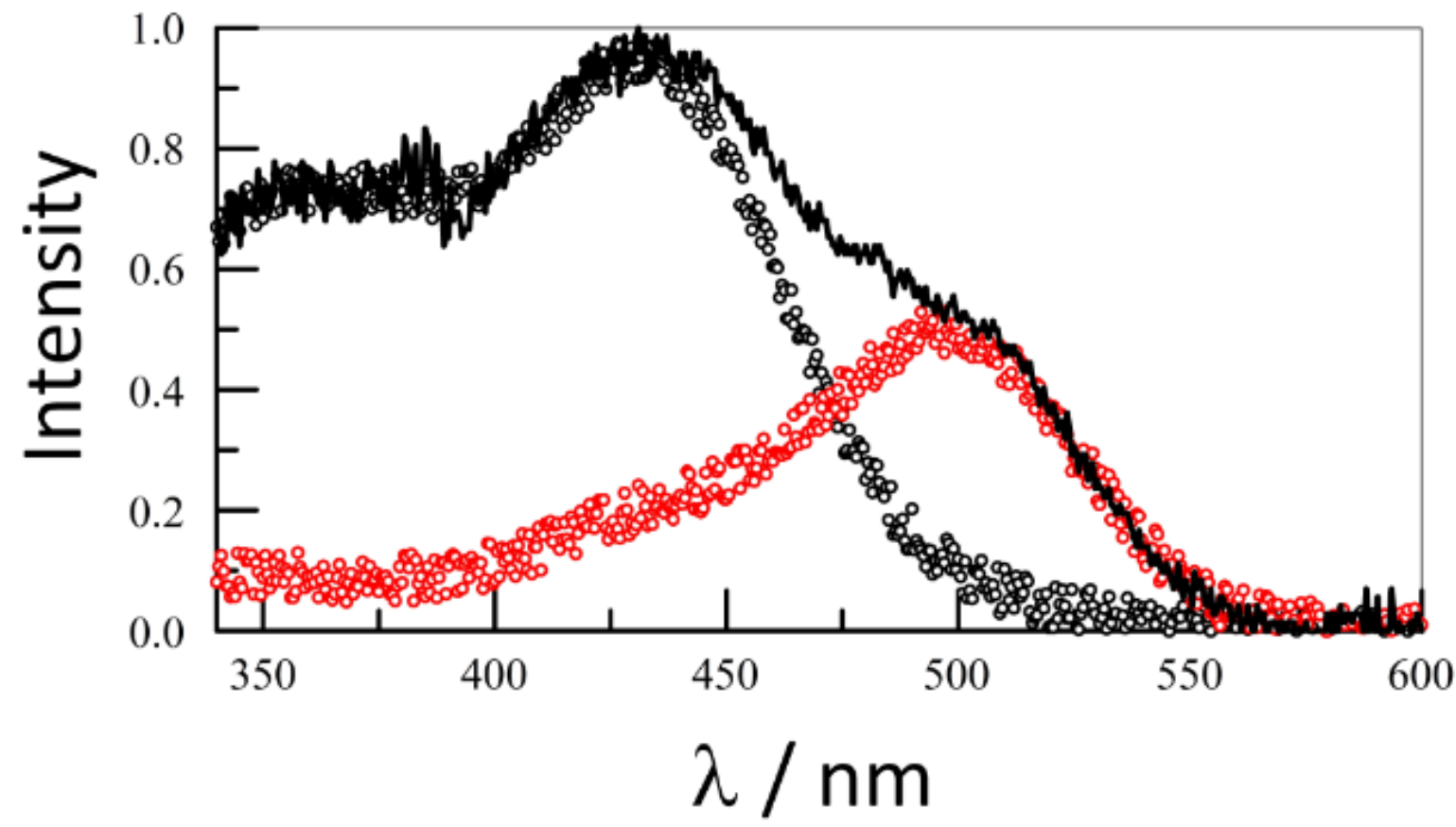

Figure S33. Comparison of normalized absorption (black circles) and excitation (open circles) spectra recorded for crystals of $\mathrm{B}\left(\mathrm{NO}_{2}\right)$ at room temperature. The emission wavelength was either $620 \mathrm{~nm}$ (red circles) or $515 \mathrm{~nm}$ (black circles). The excitation spectrum illustrated as black circles is attributed to the so-called Frenkel transition. The excitation spectrum shown as red circles is gained to either an intermolecular charge-transfer state or an aggregated state that remains fluorescent. 

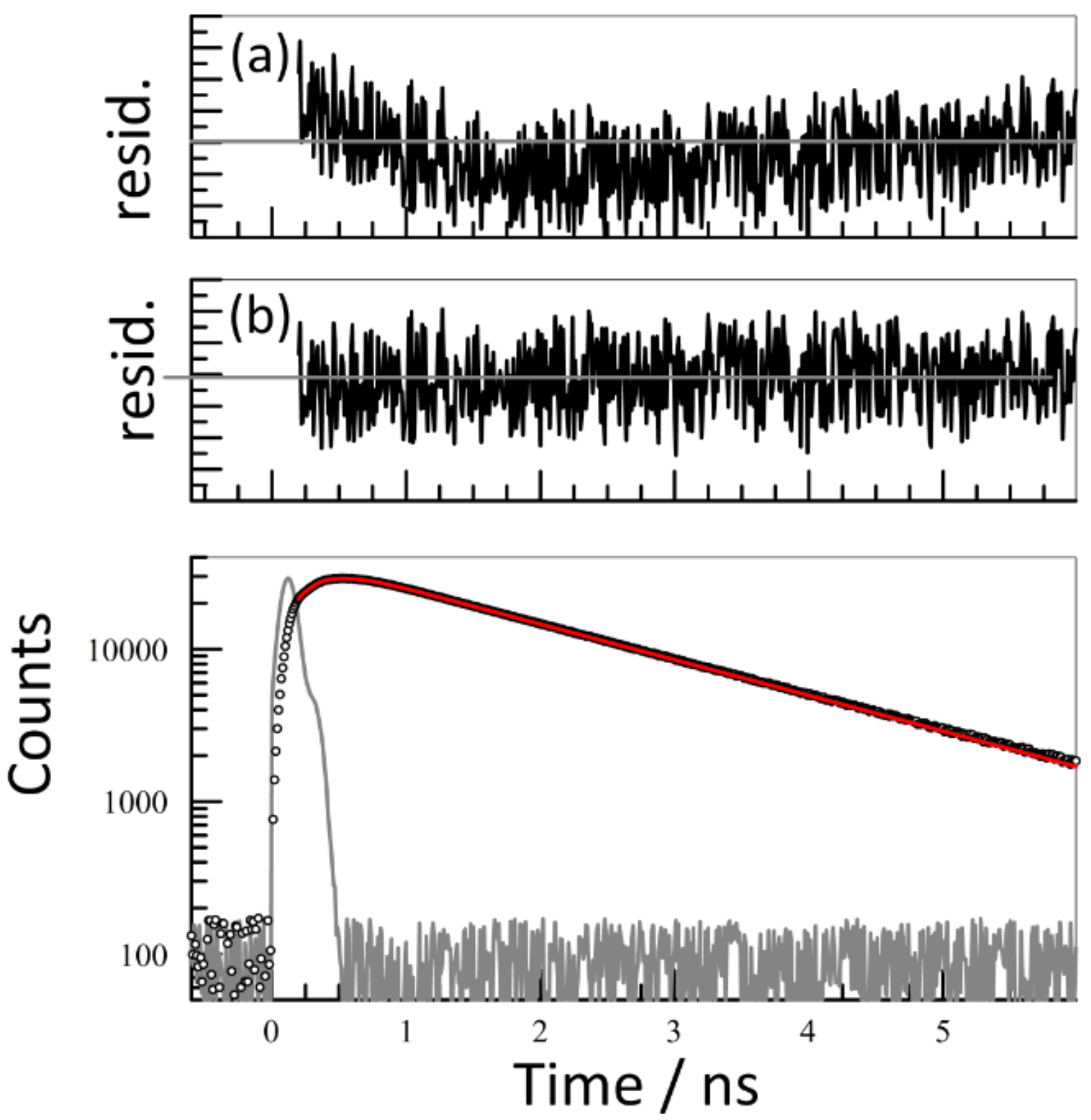

Figure S34. The lower panel shows an example of a time-resolved fluorescence decay curve recorded for crystalline $B(H)$ following excitation at $375 \mathrm{~nm}$. The instrument response function is shown as a grey curve. The fluorescence signal recorded at $500 \pm 15 \mathrm{~nm}$ is presented as open circles while the non-linear, least-squares fit is shown as a red curve superimposed over the experimental data. The fit corresponds to a stretched exponential decay with a mean lifetime of $1.80 \mathrm{~ns}$ and a stretching coefficient of 0.97 . The weighted residuals for this fit are shown as panel (b) while the residuals for a best fit to a singleexponential ( $\tau=1.87 \mathrm{~ns}$ ) fit is given as panel (a). 

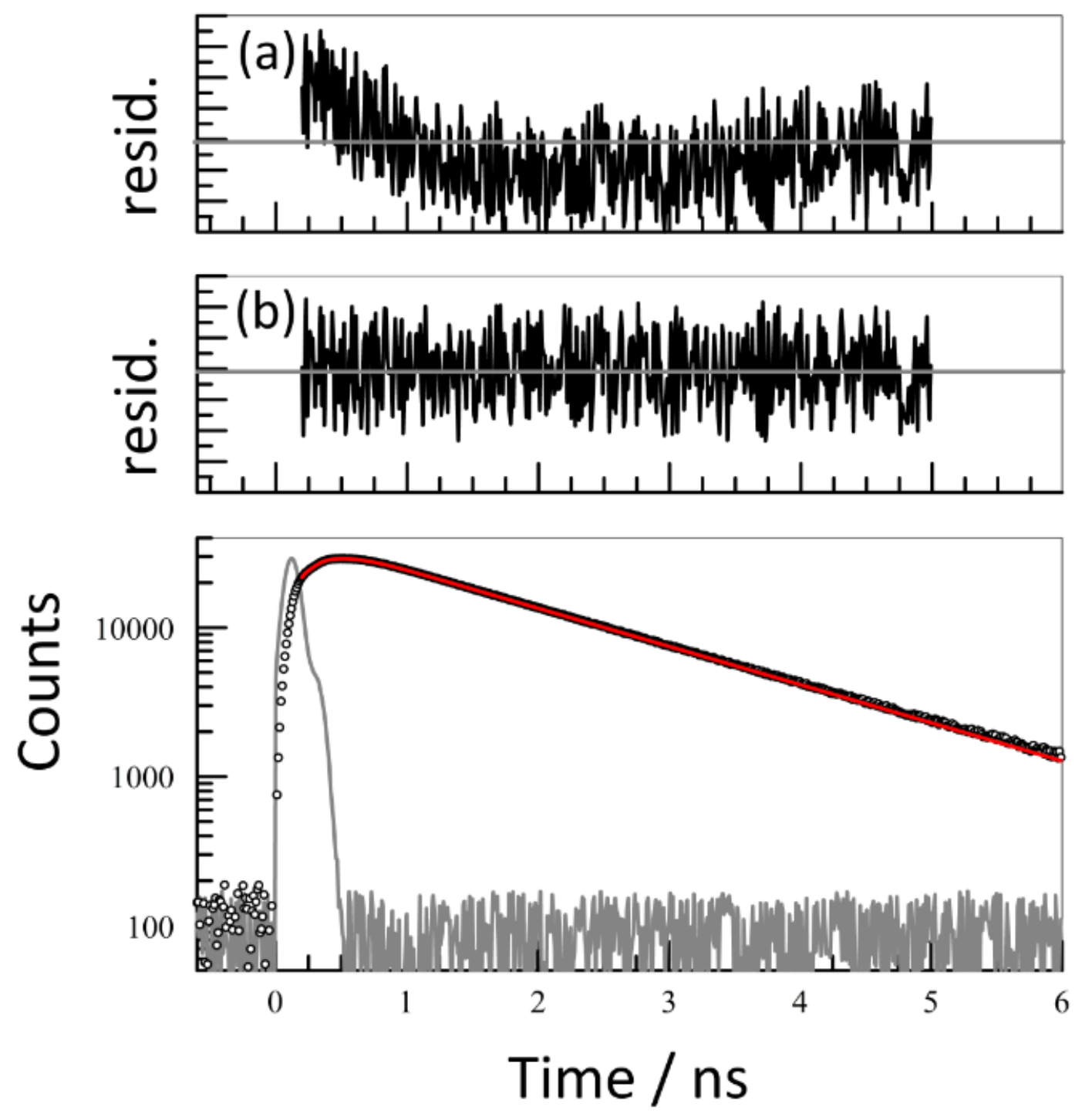

Figure S35. The lower panel shows an example of a time-resolved fluorescence decay curve recorded for crystalline $B(I)$ following excitation at $375 \mathrm{~nm}$. The instrument response function is shown as a grey curve. The fluorescence signal recorded at $480 \pm 20 \mathrm{~nm}$ is presented as open circles while the non-linear, least-squares fit is shown as a red curve superimposed over the experimental data. The fit corresponds to a stretched exponential decay with a mean lifetime of $1.60 \mathrm{~ns}$ and a stretching coefficient of 0.95 . The weighted residuals for this fit are shown as panel (b) while the residuals for a best fit to a single-exponential ( $\tau=1.71 \mathrm{~ns}$ ) fit is given as panel (a). 

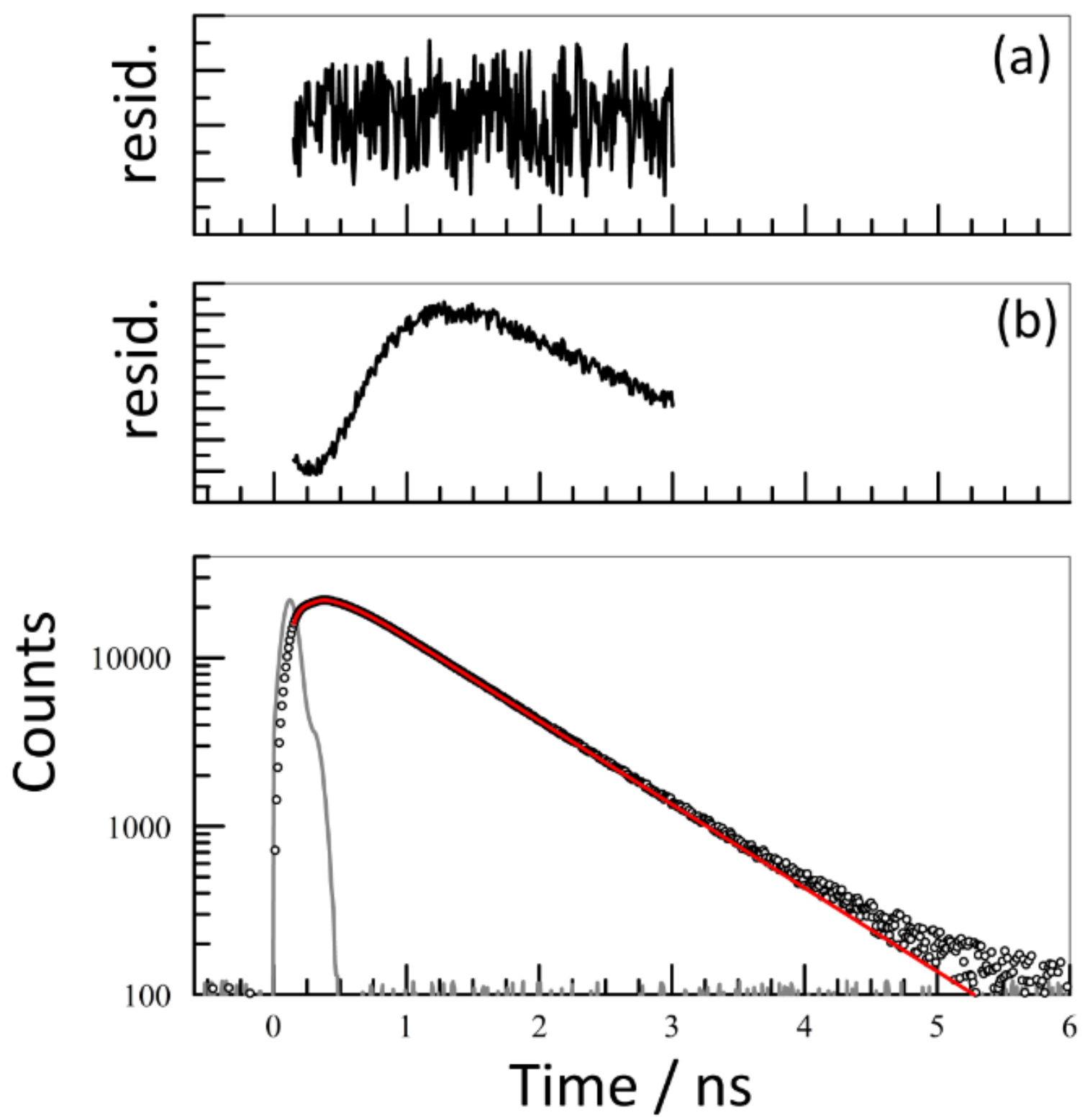

Figure S36. The lower panel shows an example of a time-resolved fluorescence decay curve recorded for crystalline $\mathrm{B}\left(\mathrm{NO}_{2}\right)$ following excitation at $420 \mathrm{~nm}$. The instrument response function is shown as a grey curve. The fluorescence signal recorded at $480 \pm 12 \mathrm{~nm}$ is presented as open circles while the non-linear, least-squares fit is shown as a red curve superimposed over the experimental data. The fit corresponds to a stretched exponential decay with a mean lifetime of 0.84 ns and a stretching coefficient of 0.96 , together with a minor component ( $11 \%$ of total) having a short lifetime of $0.18 \mathrm{~ns}$. The weighted residuals for this fit are shown as panel (a) while the residuals for a best fit to a single-exponential $(\tau=0.78$ ns) fit is given as panel (b). 

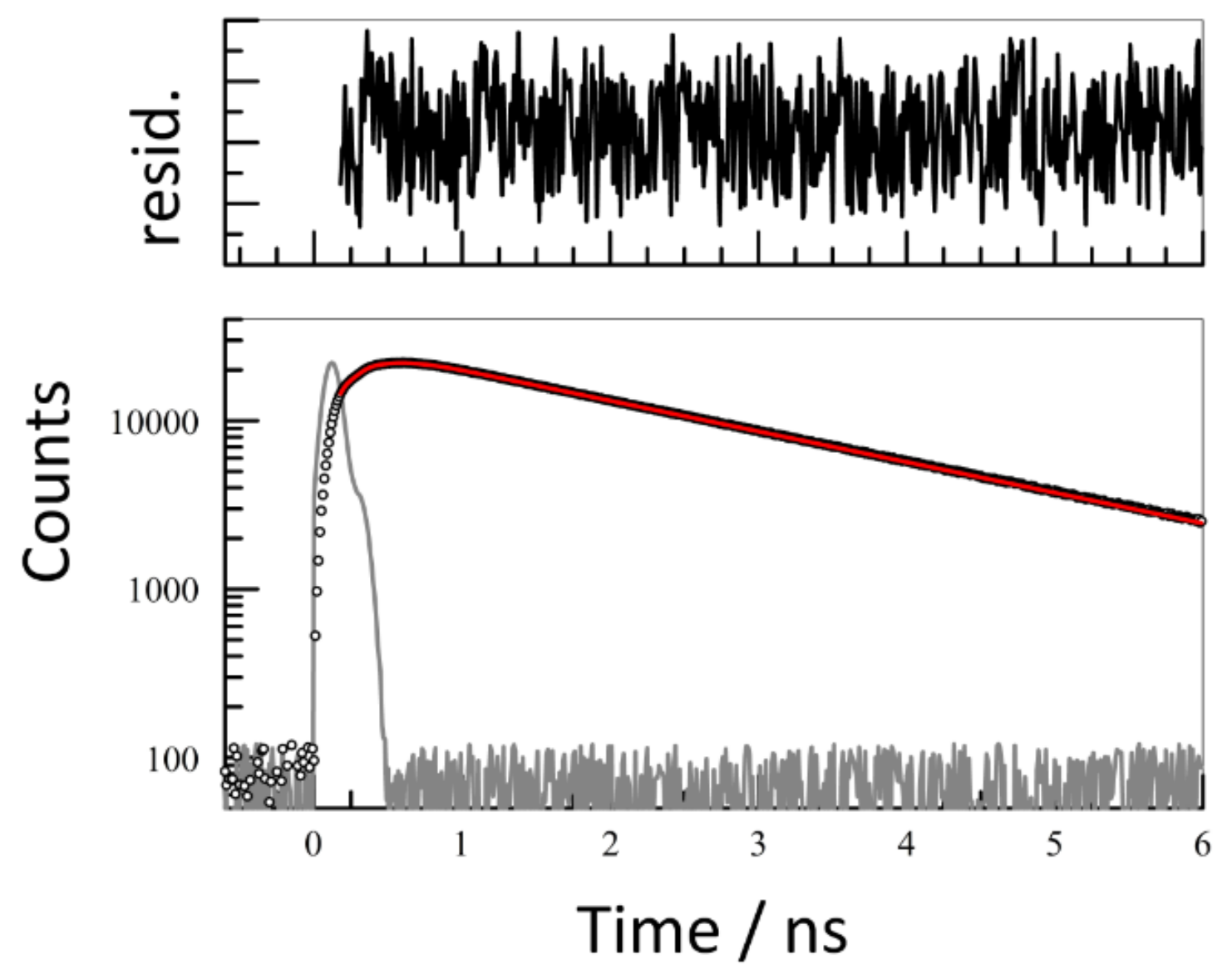

Figure S37. The lower panel shows an example of a time-resolved fluorescence decay curve recorded for crystalline $\mathrm{B}\left(\mathrm{NO}_{2}\right)$ following excitation at $515 \mathrm{~nm}$. The instrument response function is shown as a grey curve. The fluorescence signal recorded at $580 \pm 20 \mathrm{~nm}$ is presented as open circles while the non-linear, least-squares fit is shown as a red curve superimposed over the experimental data. The fit corresponds to a stretched exponential decay with a mean lifetime of 2.1 ns and a stretching coefficient of 0.88 . The weighted residuals for this fit are shown as the upper panel. 


\section{S6. Electronic energy transfer in crystalline media.}

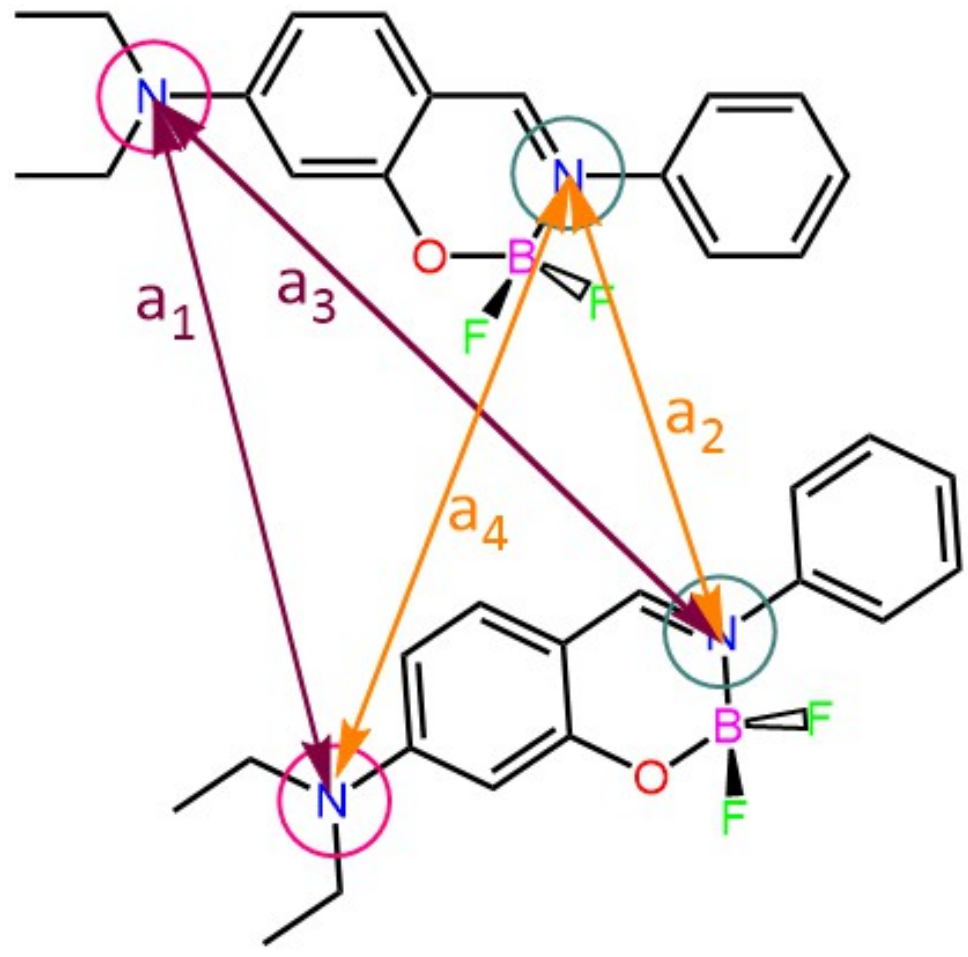

Figure S38. Pictorial representation of the geometrical parameters associated with the Kuhn extended dipole model for electronic energy transfer as applied to crystalline samples of the boranils.

We have raised the possibility that excitons generated at the interior of the crystal can migrate between neighboring molecules by way of dipole-dipole electronic energy transfer (EET). The rate of EET occurring via this mechanism depends on the distance and orientation of mutual transition dipole moment vectors. Such information can be collected for crystalline samples but data processing is tedious. The model can be simplified somewhat using the Kuhn Coulombic interaction model developed to account for excitonic interactions between closely spaced cyanine molecules. ${ }^{\mathrm{S} 19}$ The starting point for the Kuhn extended dipole approach is to identify two atoms associated with the chromophore that can be assigned to the start and finish of the transition dipole moment vector. This is easily done for the boranils by making the crude assumption that the dipole starts at the amino $\mathrm{N}$ atom and terminates at the corresponding imine $\mathrm{N}$ atom. This approximation allows easy calculation of the length $(\mathrm{I}=$ $6.366 \AA$ ) of the vector and of the Cartesian coordinates for the two $\mathrm{N}$ atoms. The second point is to derive distances (a) between relevant $\mathrm{N}$ atoms localized on pairs of proximal boranil molecules. For each pair, there will be a set of four distances that combine to characterize the 
mutual separation (Figure S38). The key advantage of this model is that it avoids the need to define orientation factors between the dipole moments in favour of the distances between termini.

The interaction integral $\left(J_{12}\right)$ between the two dipoles can be calculated from Equation $\mathrm{S} 4$, using the geometry factors obtained from crystal structures as outlined above and where $D$ is the effective screening factor imposed by the environment. The experimental transition dipole moment $\left(\mu_{\mathrm{TD}}=6 \mathrm{D}\right)$, measured from fluorescence spectra recorded in fluid solution, ${ }^{\mathrm{S} 20}$ can be used to determine the effective electronic charge $(\varepsilon)$ localized on each of the terminal $\mathrm{N}$ atoms according to Equation $\mathrm{S} 5$, where $\mathrm{I}$ is the dipole length. The rate constant ( $\left.\mathrm{k}_{\mathrm{EET}}\right)$ for EET between molecules within the pair is then calculated from Equation $\mathrm{S} 6$ where JDA is the spectral overlap integral. The latter must be calculated from normalized absorption and fluorescence spectra recorded for crystalline samples of the boranil. In this way, $\mathrm{k}_{\mathrm{EET}}$ can be approximated for each pair of reactant molecules that can be identified with a particular boranil. This situation is illustrated in Figure S39 for $B(H)$, where we identify a total of three complementary EET steps competing at the first stage. Fluorescence is assumed to occur with the same radiative rate constant as observed in fluid solution (i.e., $k_{\text {RAD }}=3 \times 10^{8} \mathrm{~s}^{-1}$ ).

$$
\begin{gathered}
J_{12}=\frac{\epsilon^{2}}{D}\left(\frac{1}{a_{1}}+\frac{1}{a_{2}}-\frac{1}{a_{3}}-\frac{1}{a_{4}}\right)=\frac{\epsilon^{2}}{D} R \\
\epsilon l=\mu_{T D} \quad(S 5) \\
k_{E E T}=\frac{2 \pi}{\hbar}\left|J_{12}\right|^{2} J_{D A}
\end{gathered}
$$

For crystalline $B(H)$, normalized absorption and fluorescence spectra can be used ${ }^{521}$ to determine a value for $\mathrm{J}_{\mathrm{DA}}$ of $0.0017 \mathrm{~cm}$, according to Equation $\mathrm{S7}$, which is deemed to be relatively high. Here, $\varepsilon(v)$ refers to the molar absorption coefficient at wavenumber $v$ and for this we take an arbitrary value of unity at the maximum. Since the integral is normalized to unity, the maximum value becomes unimportant for the calculation. The integral of the emission at wavenumber $v(F(v))$ is also normalized to unity. This leads to the generic value for $J_{D A}$, which is assumed to hold for all types of EET within the crystal lattice. Using this value, we reach the situation expressed in Equation S8, where $\mathrm{R}$ is the Kuhn geometry factor taken from Equation S4 and expressed in units of $\mathrm{m}^{-1}$. 


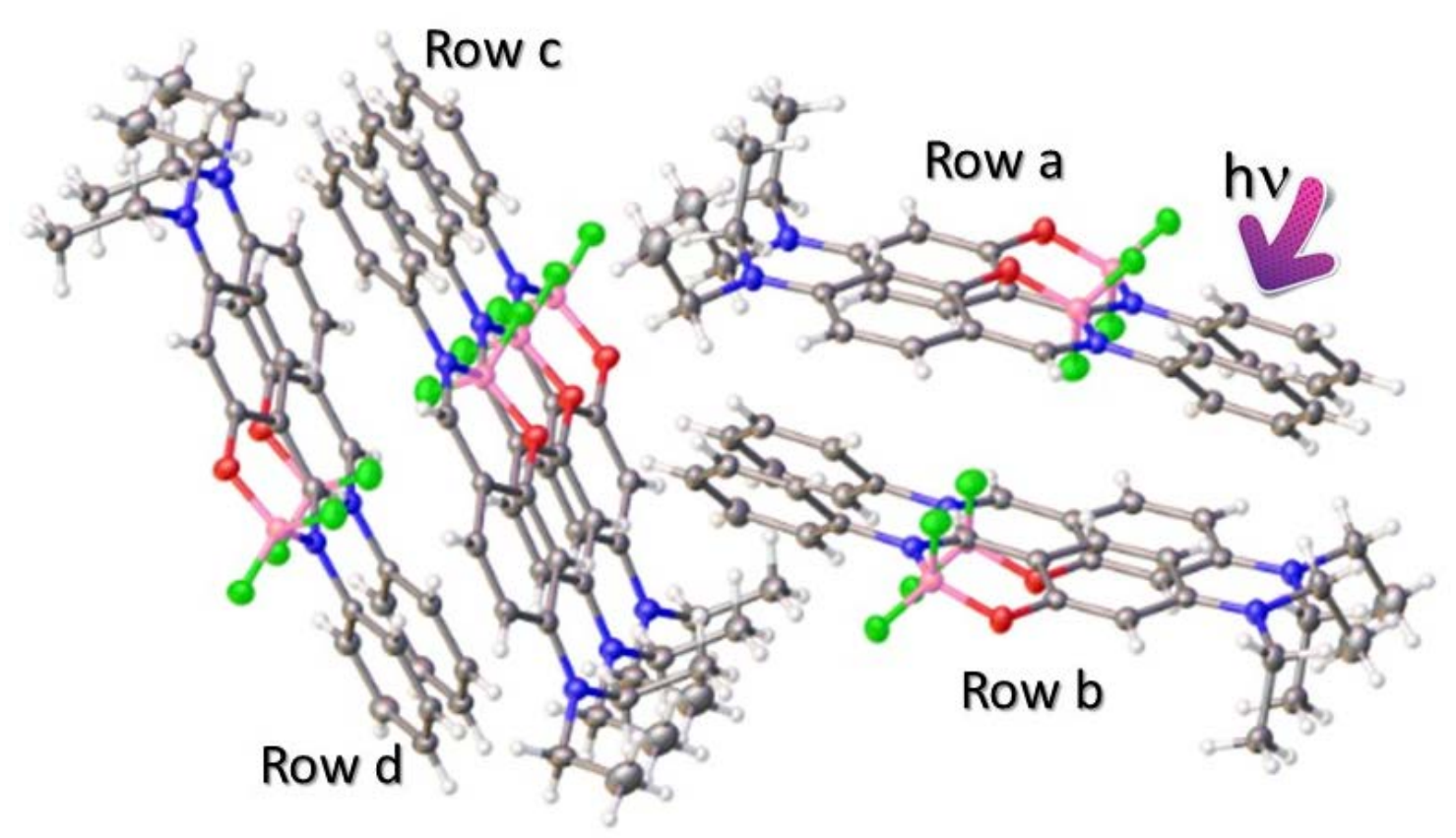

Figure S39. Illustration of electronic energy transfer pathways available for an exciton generated inside a crystal of $\mathrm{B}(\mathrm{H})$ following excitation at $390 \mathrm{~nm}$. The model assumes monophotonic excitation of a molecule associated with Row a, as shown above. Energy migration can occur between adjacent molecules aligned in Row a, or cross to a molecule associated with Row b. We consider that molecules in the head-to-head Row a are identical such that the exciton has equal probability to move in either direction along the row. We refer to this step as "head-to-head" transfer. Exciton migration to Row b could occur to either of two molecules because of the unsymmetrical spacing. Both transfers need to be considered. We refer to these steps as "head-to-tail" transfer. Row c is almost orthogonal with respect to Row a, but we can also consider exciton migration along this path. Again, two acceptor molecules need to be considered. We refer to this process as "orthogonal transfer". Once the exciton has reached Row c, "head-to-head" migration can occur to disperse the exciton within the row while transfer to Row $d$ is a further possibility.

$$
\begin{gathered}
J_{D A}=A B \int \frac{f_{D}(v)}{v^{3}} \frac{\varepsilon_{A}(v)}{v} d v \\
A \int \frac{f_{D}(v)}{v^{3}} d v=B \int \frac{\varepsilon_{A}(v)}{v} d v=1 \\
k_{E E T} \approx 9000 \times R
\end{gathered}
$$


The respective distances, and related $\mathrm{R}$ factors, obtained from the crystal structure determined for $\mathrm{B}(\mathrm{H})$ are collected in Table S8. These are referenced according to the likely EET pathways identified in Figure S39 above. It can be seen that EET within a head-to-hear row is likely to be fast, with a mean hopping time of $0.1 \mathrm{ps,} \mathrm{but} \mathrm{transfer} \mathrm{to} \mathrm{an} \mathrm{adjacent} \mathrm{head-}$ to-tail molecule is almost as likely. In fact, the overall geometry indicates an almost equal probability for EET along row a or between rows $a$ and $b$. These EET routes will conserve the molecular polarization and, at a crude level, the exciton can sample around 1,000 sites within its normal lifetime. Crossing to an orthogonal site is less likely but will still contribute to dispersal of the exciton.

Table S8. Summary of Kuhn extended dipole distances for $\mathrm{B}(\mathrm{H})$ as determined from the $\mathrm{X}$-ray crystal structure.

\begin{tabular}{|c|c|c|c|c|c|}
\hline Process & $\mathrm{a}_{1} / \AA$ & $\mathrm{a}_{2} / \AA$ & $\mathrm{a}_{3} / \AA$ & $\mathrm{a}_{4} / \AA$ & $\mathrm{R} / 10^{8} \mathrm{~m}^{-1}$ \\
\hline Head-to-head & 6.3323 & 6.3323 & 9.0938 & 8.8622 & 9.30 \\
\hline Head-to-tail & 6.6845 & 5.9205 & 11.634 & 6.6845 & 8.29 \\
\hline Head-to-tail & 6.1110 & 6.1110 & 11.406 & 5.0631 & 4.21 \\
\hline Orthogonal & 5.0158 & 11.2053 & 7.2441 & 10.4712 & 5.51 \\
\hline Orthogonal & 8.1862 & 13.0190 & 9.8189 & 12.4764 & 1.70 \\
\hline Orthogonal & 7.9687 & 12.7209 & 9.4202 & 11.9929 & 1.46 \\
\hline
\end{tabular}




\section{S7. Effect of increased temperature for a thin PMMA film.}

A thin PMMA film was cast from anisole containing $B(I)$ and dried under vacuum. The dried film was housed in the sample chamber of a Harrick's demountable liquid cell equipped with a temperature controller and a thermocouple in direct contact with the film. The cell was purged with $\mathrm{N}_{2}$ and sealed before being placed in a Hitachi F-4500 fluorescence spectrophotometer. Fluorescence spectra were recorded at different temperature, allowing 20 minutes equilibration time at each temperature. Spectra were integrated in order to monitor the effect of increased temperature on the fluorescence quantum yield. Results are presented below as Figure S40.

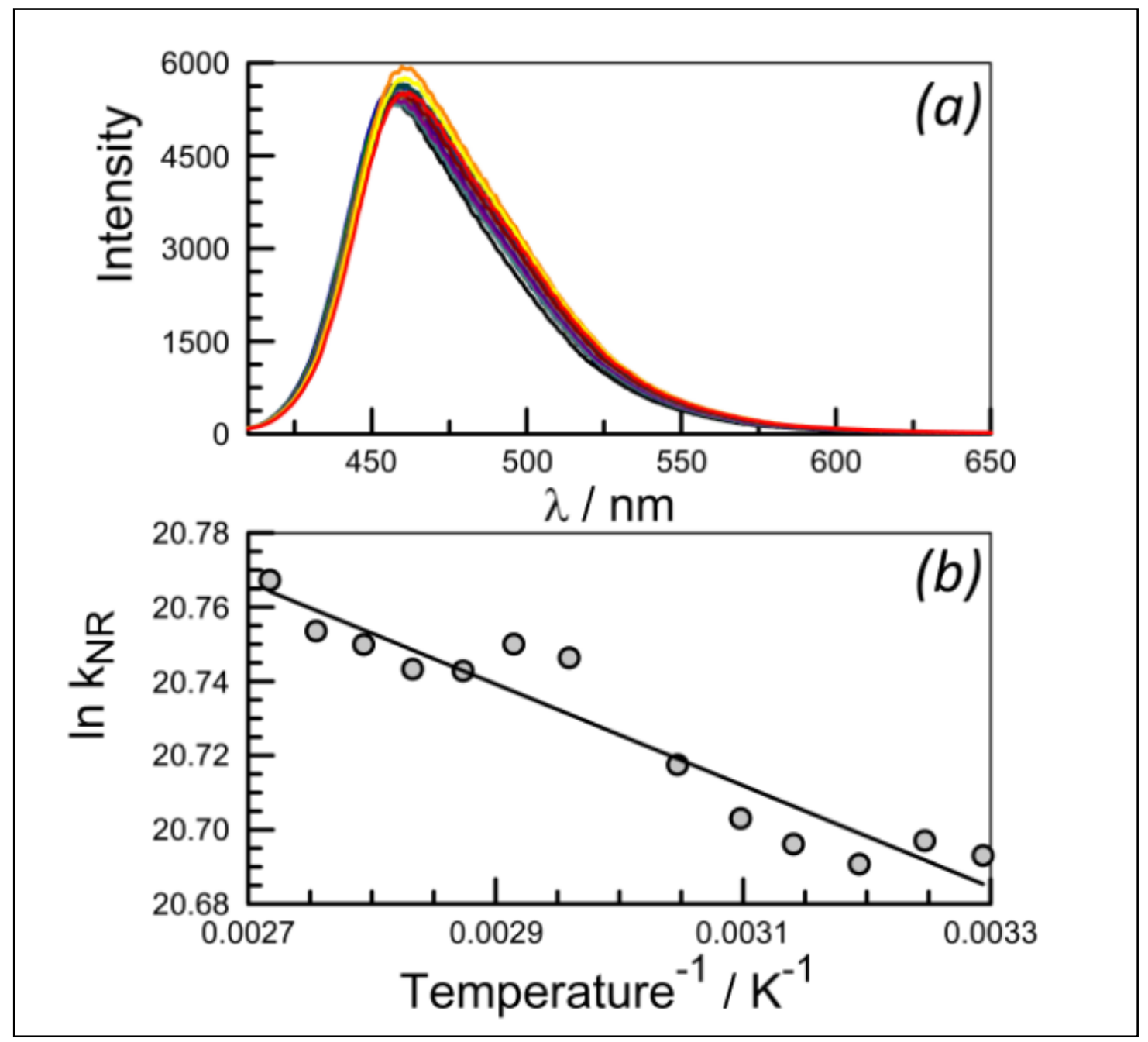

Figure S40. (a) Effect of increased temperature on the emission spectrum recorded for $B(I)$ dispersed in a thin film of PMMA. (b) Arrhenius plot constructed from the data presented in (a). The solid line drawn through the data points corresponds to a non-linear least-squares fit to the Arrhenius expression with a pre-exponential factor of $1.5 \times 10^{9} \mathrm{~s}^{-1}$ and an activation energy of $1.1 \mathrm{~kJ} / \mathrm{mol}$. 


\section{S8. Drop cast films}
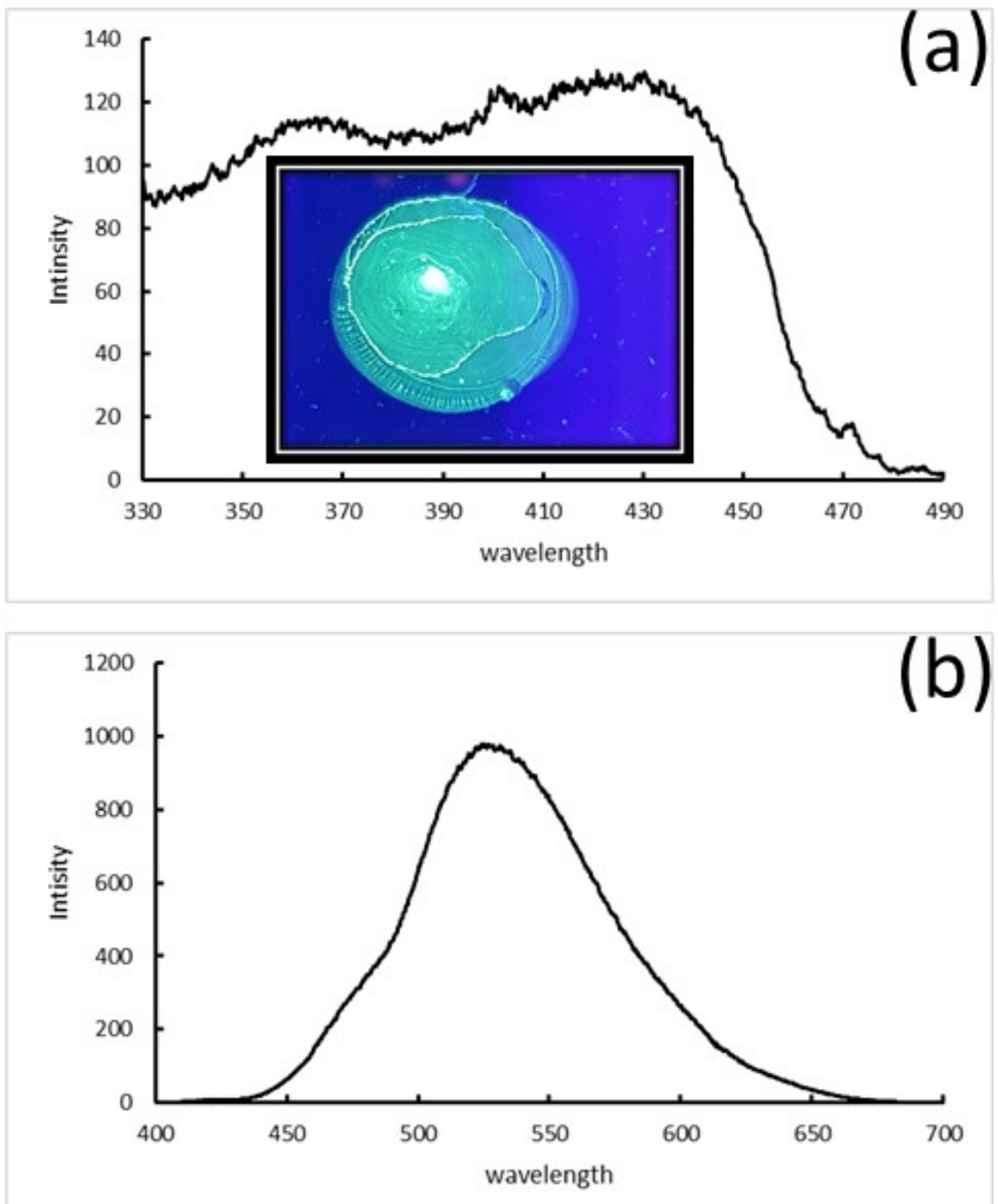

Figure S41. (a) Excitation spectrum recorded for emission from a drop cast film of LB(N), with the fluorescence wavelength being $600 \mathrm{~nm}$. (b) Fluorescence spectrum recorded for the drop cast film following excitation at $390 \mathrm{~nm}$. Emission around $480 \mathrm{~nm}$ is probably due to a monomer species while the more intense emission seen at longer wavelength is attributed to an aggregated species. The insert shows the drop cast film under illumination at $365 \mathrm{~nm}$. The film was simply cast on a microscope slide by dropping aliquots of a concentrated solution of $B(H)$ onto the warm slide. 

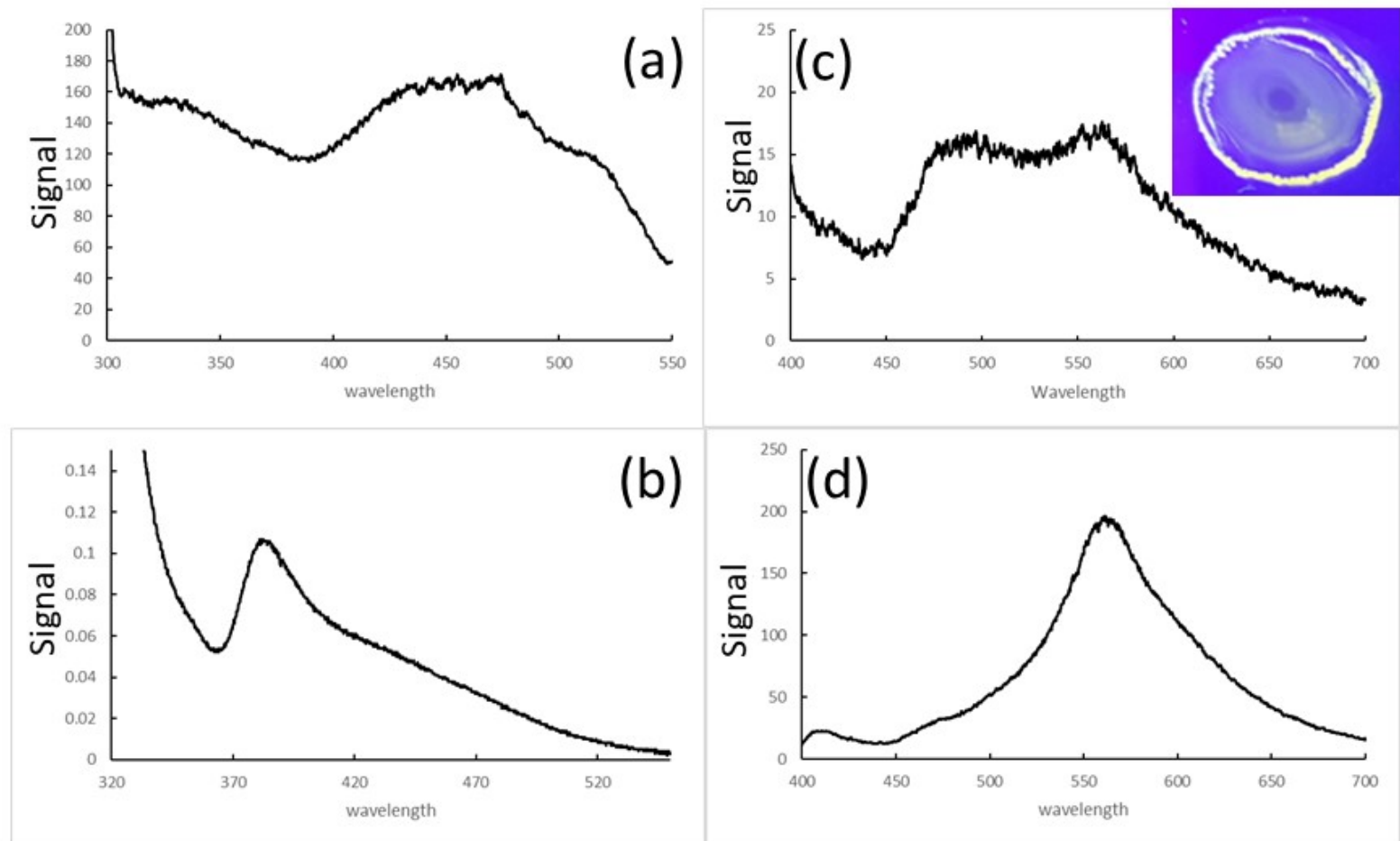

Figure S42. Results of spectroscopic studies made with a drop cast film of $\mathrm{B}\left(\mathrm{NO}_{2}\right)$. (a) Excitation spectrum recorded for emission at $600 \mathrm{~nm}$. (b) Absorption spectrum recorded for the drop cast film. (c) Fluorescence spectrum recorded from the central region. (d) Fluorescence spectrum recorded for the outer region where the chromophore is concentrated. The insert shows the drop cast film under near-UV illumination at $366 \mathrm{~nm}$. 


\section{S9. References for Supporting Information.}

(S1) Frath, D.; Azizi, S.; Ulrich, G.; Retailleau, P.; Ziessel, R. Facile Synthesis of Highly Fluorescent Boranil Complexes, Org. Lett. 2011, 13, 3414-3417.

(S2) Woodford, O. J.; Ziessel, R.; Harriman, A.; Wills, C.; Alsimaree, A. A.; Knight, J. G. Optical Spectroscopic Properties Recorded for Simple BOPHY Dyes in Condensed Media: The Mirror-Symmetry Factor, Spectrochim. Acta Part A Mol. Biomol. Spectrosc. 2019, 208, 57-64.

(S3) Brouwer, A. M. Standards for Photoluminescence Quantum Yield Measurements in Solution, Pure Appl. Chem. 2011, 83, 2213-2228.

(S4) Jones, G.; Jackson, W. R.; Choi, C. Y.; Bergmark, W. R. Solvent Effects on Emission Yield and Lifetime for Coumarin Dyes - Requirements for a Rotatory Decay Mechanism, J. Phys. Chem. 1985, 89, 294-300.

(S5) Knox, R. S. Dipole and Oscillator Strengths of Chromophores in Solution, Photochem. Photobiol. 2003, 77, 492-496.

(S6) O'Connor D. V.; Phillips, D. Time Correlated Single Photon Counting. Academic Press; London, 1984.

(S7) Porres, L.; Holland, A.; Palsson, L. O.; Monkman, A. P.; Kemp, C.; Beeby, A. Absolute Measurements of Photoluminescence Quantum Yields of Solutions Using an Integrating Sphere, J. Fluoresc. 2006, 16, 267-272.

(S8) CrysAlisPro, Rigaku Oxford Diffraction, Tokyo, Japan.

(S9) Sheldrick, G. M. SHELXT - Integrated Space-Group and Crystal-Structure Determination, Acta Crystallogr. Sect. A 2015, 71, 3-8.

(S10) Sheldrick, G. M. A Short History of SHELX, Acta Crystallogr. Sect. A 2008, 64, 112122

(S11) Dolomanov, O. V.; Bourhis, L. J.; Gildea, R. J.; Howard, J. A. K.; Puschmann, H. OLEX2: A Complete Structure Solution, Refinement and Analysis Program, J. Appl. Crystallogr. 2009, $42,339-341$.

(S12) TURBOMOLE V7.0 2015, A Development of University of Karlsruhe and Forschungszentrum Karlsruhe GmbH, 1989-2007, TURBOMOLE GmbH, since 2007; available from http://www.turbomole.com. 
(S13) Mennucci, B.; Tomasi, J. Continuum Solvation Models: A New Approach to the Problem of Solute's Charge Distribution and Cavity Boundaries, J. Chem. Phys. 1997, 106, 5151-5158.

(S14) Tomasi, J.; Persico, M. Molecular Interactions in Solution: An Overview of Methods Based on Continuous Distributions of the Solvent, Chem. Rev. 1994, 94, 2027-2094.

(S15) Al-Aqar, R.; Benniston, A. C.; Harriman, A.; Perks, T. Structural Dynamics and Barrier Crossing Observed for a Fluorescent O-Doped Polycyclic Aromatic Hydrocarbon, ChemPhotoChem 2017, 1, 198-205.

(S16) Hickey, A. L.; Rowley, C. N. Benchmarking Quantum Chemical Methods for the Calculation of Molecular Dipole Moments and Polarizabilities, J. Phys. Chem. A 2014, 118, 3678-3687.

(S17) Abboud, J.-L. M.; Notari, R. Critical Compilation of Scales of Solvent Parameters. Part 1. Pure, Non-Hydrogen Bond Donor Solvents, Pure Appl. Chem. 1999, 71, 645-718.

(S18) Dobkowski, J.; Wnuk, P.; Buczynska, J.; Pszona, M.; Orzanowska, G.; Frath, D.; Ulrich, G.; Massue, J.; Mosquera-Vazquez, S.; Vauthey, E. Substituent and Solvent Effects on the Excited State Deactivation Channels in Anils and Boranils, Chem. Eur. J. 2015, 21, 1312-1327.

(S19) Czikklely, V.; Forsterling, H. D.; Kuhn, H. Extended Dipole Model for Aggregates of Dye Molecules, Chem. Phys. Lett. 1970, 6, 207-210.

(S20) Lambert, C.; Scherpf, T.; Ceymann, H.; Schmiedel, A.; Holzapfel, M. Coupled Oscillators for Tuning Fluorescence Properties of Squaraine Dyes, J. Am. Chem. Soc. 2015, 137, 3547-3557.

(S21) Harriman, A.; Ziessel, R. Exploring the Effects of Solvent Polarity on the Rate of Förster-Type Electronic Energy Transfer in a Closely-Spaced Molecular Dyad, Photochem. Photobiol. Sci. 2010, 9, 960-967. 